\title{
Sismicità in relazione alla tettonica e accumulo delle tensioni elastiche nella regione abruzzese, nel periodo 1900-1970
}

\author{
(Seismicity referred to the tectonics and the accumulation of elastic tensions \\ in the Abruzzi Region in the period between 1900 and 1970)
}

\author{
P. Caloi - U. Colladonato - M. C. Spadea
}

Ricevuto il 5 Oıtobre 1969

Riassunto. - Lo studio ivi riassunto si riferisce alla sismicita della regione abruzzese (intesa in senso lato) e riguarda il periodo 1900-1970. I terremoti verificatisi in tale periodo, di intensità superiore al III grado della Scala Mercalli, furono 380 circa. Pochi di essi, per evidenti motivi, sono stati studiati con i metodi microsismici: la grande maggioranza è stata sottoposta ad indagini macrosismiche, intese al rilevamento delle zone epicentrali, della profondità ipocentralo e della magnitudo. Naturalmente, per tale via, il rigore quantitativo non ha ragion d'essere. Piuttosto, ci si è preoccupati di avvalersi di metodi unitari, cosi da consentire un accettabile valore relativo dei risultati. D'altronde, specie per quanto concerne la magnitudo - grandezza, definita entro larghi margini e soggetta a notevole variabilità di interpretazione - , sarebbe illusorio la ricerea di approssinazioni spinte.

Terremoti di magnitudo superiore a 6 si verificarono nelle seguenti località: zona del Velino 6.6 (1904); Avezzano 7.5 (1915); Arquata del Tronto 6.2 e Cittareale 6.3 (1916); Acquaviva d'Isernia 6.2 (1925); I ama dei Peligni 7.0 (1933); Gran Sasso d'Italia 7.1 (1959); Monti della Laga 6.5 (1951); L'Aquila 6.2 (1958); Sorgenti del Peschiera 6.4 (1961).

I'attività sismica fu massima lungo l'allineamento Vinchiaturo Isernia - Avezzano - Monte Velino - Sorgenti del Peschiera, che coincide con la linea tettonica più attiva (ivi si verificarono il terremoto disastroso di Avezzano (1915), i forti terremoti del Velino (1904), di Acquaviva d'Isernia (1925, ecc.); fortissima a SE della Maiella con i rovinosi terremoti di L ama dej Peligni (1933), Taranta Peligna, ecc.; fortissima fra il Gran Sasso e i Monti della Isaga. I e profondità ipocentrali furono generalmente 
piccole: dell'ordine di $15 \mathrm{~km}$ quetla del terremoto di Avezzano, la massima profondità non avendo superato i $25 \mathrm{~km}$.

Nella 111"a parte del lavoro si fa un ampio esame della sismicitì in relazione alla tettonica regionale. Per quanto concerne il meccanismo dei terremoti all'ipocentro, si riscontra che la spinta verso l'esterno (compressione), che ha caratterizzato i terremoti del Gran Sasso e dei Monti della Laga, coincide con i movimenti tettonici che hanno originato i massicci montani in questione, come da conclusioni di Fabiani - Segre - Demangeot. Fra gia st ato sottolineato il contrasto fra i terremoti appenninici, caratterizzati da compressioni all'ipocentro, con i terremoti dell alto Adriatico, in cui predominano le dilatazioni ipocentrali: orogenesi ancora in atto nel prino caso, flessioni associate alla fossa adrio-jonica nel secondo. Ma le due zone presentano altri contrasti strutturali. Nel 1937 era stato provato che le onde trasversali subcrostali dell'Alto Adriatico si propagano con una velocitì di $4.8 \mathrm{~km} / \mathrm{sec}$., nettamente superiore alla media. Ciò presupponeva uua deficienza di massa a favore della rigidità. Una ricerca del giapponese Kawabata (1954) sulle variazioni della verticale, in Italia, e zone limitrofe, mostrava nette deviazioni verso gli Appennini e le Alpi Dinariche: in altri termini, anche per questa via, risultava per l'Adriatico una densitì della crosta sensibilmente inferiore a quella corrispondente agli Appennini e alle Alpi Dinariche, a conferma di un contrasto strutturale profondo fra le zone interessate.

Nella parte quarta si traccia l'andamento (valevole, naturalmente, solo come indicazione approssimativa) dell'accumulo delle tensioni elastiche nelle zone soggette a deformazione, sfociata successivamente in manifestazioni sismiche. Gli intervalli di tempo caratterizzati da relativa calma sismica, variano nel settantennio fra gli undici e i diciassette anni circa. Dal 1961 è in atto un periodo di relativa quiete. Uno scarico di energia in fase di ac. cumulo mediante esplosioni nucleari sotterranee nella zona Gran Sasso Fucino, sarebbe quanto mai propizio. Purtroppo, interventi del genere sono aucora inattuabili.

Sumarx. - The here summarized study deals with the seismicity of the Abruzzi Region (understood in a larger meaning), as observed in the 1900-1970 period. The number of earthquakes with higher intensities than Grade III of the Mercalli Scale was about 380. For obvious reasons only a few of them have been studied by microseismic methods. Their great majority has been subjected to macroseiemic research work in order to locate the epicentral areas, the depths of epicentres and to ascertain the magnitude. This research work could not be quantitatively rigorous, of course. Its concern lay, rather, in the use of unitarian methods; so as to achieve an acceptable relative value of the results. On the other hand, there could he no illusions of arriving at far reaching approximations, especially as far as magnitude -- size is concerned, which had been defined within wide margins and subjected to a considerable variability of interpretation.

Earthquakes with magnitudes larger than 6 have been recorded in the following spots: the Velino area 6.6 (1904); Avezzano 7.5 (1915); Arquata del 'Tronto 6.2 and (ittareale 6.3 (1916); Acquaviva d'Isernia 6.2 (1925); 
Lama dei Peligni 7.0 (1933); Gran Sasso d'Italia 7.1 (1959); the Laga Mountains 6.5 (1951); L'Aquila 6.2 (1958); the Peschiera Springs 6.4 (1961).

'The seismic activity was highest along the conjunction line Viuchiaturo - Isernia - Avezzano - Monte Velino - Peschiera Springs, which coincides with the line of major tectonic activity (locations of the terrific $A$ vezzano earthquake of 1915, the strong earthquakes of Velino in 1904 and of Acquaviva d'Isernia in 1925, etc.; the tectonic line is strongly eviclenced to the SE of the Maiella Massive where the ruinons earthquakes of Lama dei Peligni (1933), Taranta l'eligna and others took place, as well as in the area between the Gran Sasso and the Laga Mountains). The lypocent ral depths are generally limited, that is in the order of $15 \mathrm{~km}$ for the Avezzano earthquake, with maximum depths not over $25 \mathrm{kms}$.

The IIIrd part of the study presents an ample examination of soismicity with reference to the tectonical aspect of the region. Concerning the mechanism of the earthquakes in the hypocentre, it has been found that the thrust toward outside (compression), which was the chief feature of the Gran Sasso and the Iaga Mountains earthquakes, coincides with the tectonic movements which were at the origin of the said mountain massiven, according to the conclusions of Fabiani, Segre and Demangeot.

The contrast between the earthquakes of the Apennines Region, whose main feature are the compressions at the hypocentre, and the earthquakes of the Iligh Adriatic Region where hypocentral dilatations are preclominant, has already been pointed out: in the first instance the orogenesis is still in course, and in the second there are flexions associated with the $\Lambda$ drio Jonic ditch. But these are not all the structure contrasts between the two areas. It had been shown in 1937 that the sub-crustal transversal waves of the Iligh Adriatic Region spread at the speed of $4.8 \mathrm{~km} / \mathrm{sec}$, which is clearly above the average. This required a deficiency of mass in favour of stiffness. A study conducted by the Japanese Kawabata in 1954 on the variations of the vertical in Italy and adjacent areas, ascertained outspoken deviations towards the Apennines Range and the J)inaric Alps; in other words, also through this approach a tangibly lower density of the crust in the Adriatic area in comparison with that of the Apenuines and the Dinaric Alps was brought to evidence and confirmed a deep structural contrast between the two areas.

The IVth part outlines a trend - which is only approximatively indicative - of the building up of elastic tensions in the areas subject to deformation which found successively an outlet of seismic phenomena. The time intervals of relative seismic calm vary between 11 and 17 years in the 70-year period considered. A period of relative stillness is being observed since 1961 . The discharge of upbuilding energy through nuclear underground blasts in the Gran Sasso - Fucino area would appear more helpful than ever, but unfortunately the interventions of this kind cannot be realized as yet. 


\section{I. - LiNeayexti TETTONiCI DELLA zONA LAZIALE-ABRUZzese, CON PAR- TICOLARE RIGUARDo ALL'ABRUZzo.}

L'interpretazione tettonica delle masse calcaree costituenti la facies laziale-abruzzese, per la complessità d'insieme, ha subito nel tempo diverse modificazioni, sempre più valide quanto più profonde sono state le indagini, alla luce di conoscenze nuove che pervenivano direttamente dallo studio e dalla ricerca. Queste nuove conoscenze non lamno investito tanto il piano stratigrafico quanto quello tettonico.

Così mentre l'interpretazione del Beneo, ad esempio, ha assegnato al complesso una tettonica di innalzamenti e sprofondamenti di tipo Hors e Graben, postulando l'esistenza di faglie inverse sul versante adriatico e faglie dirette sul versante tirrenico, l'interpretazione data dal Prof. Accordi, basata su nuovi studi stratigrafici, paleontologici, ha portato a considerazioni di tettonica traslativa, ad andamento $\mathrm{W}-\mathrm{F}$, con spostamenti sempre meno vistosi dal Tirreno all'Adriatico.

Lo studio sismologico che ci siamo proposti, in verità non investe direttamente la genesi della tettonica, ma gli effetti tettonici ed in particolare le interazioni fra le faglie e i movimenti sismici che hanno interessato ed interessano la regione. In ogni modo, poiché alcune nostre conclusioni, dovute a semplici considerazioni desunte dal problema affrontato, ci porteranno, sia pur genericamente, a delle affermazioni di carattere genetico (per quanto riguarda la tettonica), sia perchè una descrizione geologica è atta ai nostri fini, abbiamo voluto documentare questo nostro lavoro della sintesi cui sono giunti gli Autori che da ultimi si sono interessati di questa regione.

Procederemo dalle tesi del Beneo a quelle di B. Accordi, sia per rispettare una cronologia doverosa, sia perchè le teorie di quest'ultimo ci sembrano più vicine alle nostre considerazioni.

L'Appennino calcareo è compreso tra la facies Umbro-Marchigiana ed il Massiccio Calabrese: è costituito da una ossatura calcarea-mesozoica autoctona, mentre i terreni del Terziario ricoprono porzioni molto limitate. A Sud-Ovest il complesso si immerge, con un sistema di fosse e di massicci, sotto al retropaese tirrenico, a NE l'avampaese molassico continua a Sud con la formazione delle Argille scagliose e del Flysch per proseguire nella Fossa Bradanica, profonda geosinclinale del Mediterraneo orientale. In tutto il complesso quindi si riscontra la presenza di due grandi categorie di serie, una autoctona e l'altra alloctona; il nostro lavoro analizza le caratteristiche tettoniche e tenta la deter- 
minazione del meccanismo della alloctonia e la sua distribuzione geografica.

Il complesso alloctono delle "Argille Scagliose " è costituito da terreni prevalentemente argillosi appartenenti ad età diverse che vanno dal Cretaceo, fino, talvolta, al Miocene medio-superiore. La traslazione delle argille scagliose è avvenuta, in più di una ripresa, con modalità di vere grandi colate, in un ambiente quasi sempre sottomarino, corrispondente ad altrettanti periodi di stasi orogenetica. Durante tali soste si formano su di esse quei depositi "parautoctoni " che, ad una ripresa del movimento, si sposteranno con esso.

Nel versante tirrenico dell'Appennino Settentrionale, la parte più alta dell'autoctono è rappresentata dal "macigno". Poiché l'età del macigno è oligocenica, l'invasione delle argille scagliose si sarebbe iniziata dall' Oligocene. Il periodo durante il quale sono avvenute le grandi colate sembra essere terminato col Langhiano o con l'Elveziano.

Nel Lazio le argille scagliose si trovano in piccoli lembi sui calcari elveziani, il primo parautoctono, ed anche la parte più alta della serie autoctona.

In Abruzzo ad Est della Maiella e nel Molise, come in Lucania e in Basilicata, esistono $i$ medesimi rapporti osservati nel Lazio fra le argille scagliose e gli altri terreni. Anche qui il movimento avviene nel senso da ponente ad oriente.

La forza che ha originato la traslazione è quella di gravità ed in particolare le sue componenti tangenziali. La componente tangenziale di gravità è parallela in ogni punto alla superficie inclinata delle masse meno plastiche sulle quali gravano le argille scagliose. Il moto delle argille scagliose avverrà quando l'inclinazione della superficie suddetta supererà il loro angolo d'attrito. Ma affinché si esplichi la componente tangenziale di questa forza è necessaria la formazione di innalzamenti del substrato: la natura di queste forze ci è ignota. Gli innalzamenti o dislocazioni del substrato, se pure hanno ampiezze regionali, non sono né continui, né contemporanei, ma procedono nel tempo e nello spazio secondo direttrici. In conseguenza, anche la traslazione delle argille scagliose subisce degli arresti per il raggiungimento di un equilibrio causato dall'annullarsi delle componenti tangenziali.

L'autoctono, per la propria rigidità costituzionale, è caratterizzato da una tettonica ribelle al piegamento; il motivo fondamentale della struttura è la faglia con tutte le combinazioni o complicazioni dovute alla resistenza delle zolle calcaree. Le linee di disturbo tettonico si raggruppano in due sistemi: uno longitudinale, con dire- 
zione NW-SE, l'altro trasversale che interseca il precedente sotto angoli diversi. Si è spesso parlato della priorità di formazione di queste due linee, senza riuscire però a trovare argomenti inequivocabili, atti ad una sicura dimostrazione; si è optato tuttavia per la precedenza del sistema trasversale nel suo insieme.

Una eccezione a tale idea sembra fornita da un fascio di faglie trasversali costituenti una linea tettomca con direzione NE-SW, che taglia in due parti pressoché uguali la parte Nord-Orientale dell'Abruzzo.

Questa linea è facilmente ricostruibile, nonostante diverse lacune che ne impediscono la continuità. A SW la si vede al margine Settentrionale della depressione costituita dall'alveo del Fucino e ciò̀ ai Tre Monti. Sopra Celano interrompe le bancate calcaree dolomitiche sul versante Meridionale della Serra. Dopo aver attraversato la valle del Cancello, la linea scende nel fondovalle fra il monte Pelara e il monte S. Pietro, risalendo poi verso il monte $\mathrm{S}$. Nicola. Il versante NE del Sirente interessato da una potente distesa detritica, nasconde completamente i terreni che sono stati interessati dalla faglia. Poco oltre si ritrova il fascio di faglie nei calcari del vallone che sbocca a Gagliano Aterno. Procedendo ancora verso NE la faglia separa la montagna del Morrone dalle propaggini meridionali del G. Sasso. Nel versante orientale del monte Picca, come pure in quello di Morrone, ha luogo il grandioso ribaltamento delle masse calcaree sulle molasse della zona costiera adriatica. Il ribaltamento avviene a mezzo di un fascio di faglie inverse la cui superficie di scorrimento tende, talora, fino all'orizzontale. Qui la nostra linea diviene meno evidente. La linea viene a cessare oltre Pescosansonesco, venendo a cessare nelle molasse gli efietti delle forze che l'hanno prodotta.

Osserviamo ora lungo la linea trasversale, il fenomeno delle linee longitudinali tagliate dalle trasversali in due parti, sicché possiamo considerare le due linee in settentrionali e meridionali. Mentre le linee meridionali seguono il loro normale andamento $\mathrm{NW}$-SE, quelle settentrionali presentano nella vicinanza della linea trasversale una sensibile deviazione verso Nord. Questa deviazione si fa più forte procedendo dal Fucino verso Nord-Est, fino a coincidere col meridiano nel ramo sud della catena del Gran Sasso. I vertici di curvatura, che sono la conseguenza di tali deviazion, si spostano verso Nord quando si procede nel medesimo senso di prima. Lo spostamento massimo è situato al centro della catena del Gran Sasso la cui vetta risulta formata 
da due rami principali, uno con direzione EW l'altra a Sud con direzione NS.

In altri termini, tutto cio ha $i l$ significato $d i$ un perdurare $e$ di un accentuarsi locale delle forze orogenetiche che dettero, in tempi relativamente prossimi, l'attuale configurazione tettonica dell'A ppennino. Per cui la rottura nelle faglie longitudinali è dovuta alla spinta ulteriore che le masse dei terreni venivano a ricevere, creando così un movimento di traslazione da SW a NE, rispetto al già avvenuto assestamento della massa meridionale.

Molte di queste faglie sono state nel tempo soggette a ripetuti movimenti. Vi sono infatti faglie composte da più piani, più o meno paralleli, che vanno considerate non di formazione contemporanea, ma ciascuna come conseguenza di una successione di periodi di attività tettonica. Un esempio recente ̀̀ offerto dalla faglia Gioia-Sperone; parallelamente ad essa durante il macrosisma 15 gennaio 1915, si formò una frattura marginale alla pianura che si aperse e chiuse ripetutamente. Rimase riconoscibile per vari mesi per un tratto di $70 \mathrm{~km}$.

Consideriamo ora "La valle dell'Aterno " che si rivela come uno degli esempi più tipici dell'Appennino abruzzese per la impressionante sequenza di faglie a gradinata.

Ne costituiscono la stratigrafia terreni del Secondario, prevalentemente calcari, del Terziario, calcari, brecce, molasse, argille, e del Quaternario, con marne e conglomerati.

Fra le linee di frattura, sono specialmente da ricordare quelle del versante NE del Sirente, che si riallacciano a $\mathrm{NW}$ con quelle dei monti d'Ocre a SE con quelle della conca Subequana. Nella conca aquilana, prolungamento a NW della valle d'Aterno, avviene la riunione di varie fratture appartenenti ai due sistemi; questo determina la formazione di una estesa zona milonitica. La parte sinistra della valle, infine, è interessata da una serie di fratture, con lunghezze e rigetti però meno vistosi. L'età delle fratture e degli spostamenti si può determinare considerando che tutti i terreni, dalle formazioni secondarie a quelle quaternarie, ne sono interessati; poiché però il fenomeno si rivela più vistoso al di fuori dei terreni interessati dai terreni fluviolacustri del Villafranchiano, possiamo presumere che l'inizio degli spostamenti abbia avuto luogo al massimo al Pliocene; in alcune zone d'altronde il fenomeno si prolunga nel resto di questo periodo.

Sarà bene ricordare la grande faglia di Beffi-Molina in corrispondenza di Acciano. Questa faglia, che si prolunga a SE verso la conca di Sulmona e a NW verso la zona di Fagnano-S. Demetrio, fa parte di 
una serie numerosissima di faglie situate alla sinistra dell'Aterno, interessa verso SE la serie di calcari eocenici, mentre a NW interessa i calcari cretacei e la serie miocenica.

La zona di S. Demetrio, Fagnano, Fontecchio, Beffi, sulla sinistra dell'Aterno, sono interessate da un rilevante numero di faglie del sistema longitudinale; non mancano però quelle del sistema trasversale specialmente tra i territori di S. Demetrio e di Fagnano.

Se il motivo tettonico fondamentale della struttura abruzzese è la faglia, non mancano però le pieghe. Queste hanno la particolarità di essere molto blande nei versanti occidentali delle catene, e di accentuarsi sempre più, fino a veri e propri contorcimenti e stiramenti degli strati, nelle vicinanze dei bordi orientali, dove si ha una spiccata tendenza al ribaltamento. Nei Lepini esiste una piega-faglia con inclinazioni e scorrimenti crescenti da NW a SE che interessa tutto il bordo orientale, per cui i calcari del Cretaceo si sovrappongono alla formazione argilloso-arenacea miocenica della Valle Latina. Lo stesso fatto si verifica nel versante orientale del Morrone, dove però i calcari che si sovrappongono alle argille-arenarie del Miocene, appartengono sia al Cretaceo sia all'Eocene sia al Miocene stesso.

Nei Simbruim il fenomeno del ribaltamento è assai meno accentuato. Il versante orientale del Sirente, sulla destra della valle dell'Aterno, è invece un mirabile esempio di faglie multiple a gradinata. Sempre nella valle dell'Aterno il fenomeno della piega-fagha ribaltata si ripete poco più a Nord, vale a dire nel bordo orientale dei Monti d'Ocre.

E naturale che con i tipi di dislocazione descritti non manchino le fosse tettoniche. Due valli tettoniche dirette NW-SE, fiancheggiano la catena Simbruino-Ernica separandola dalla catena dei Lepini e da quella dei Marsicani; la prima è la valle del Sacco o valle Latina, la seconda è l'alta valle del Liri o val Roveto. Queste due valli, pur avendo entrambe la stessa origine, differiscono nel motivo tettonico del loro lato sinistro, che è un semplice elemento di anticlinale nella valle I atina e una imponente faglia nella valle Roveto, mentre il loro lato destro è costituito da una piega faglia più o meno inclinata. Un'altra fossa tettonica è la valle di Caramanico, fra il Morrone e la Maiella. Questa è tagliata da una serie di faglie poste sul prolungamento di quelle del bordo occidentale della Maiella.

Il gruppo della Maiella viene considerato un intenso e complesso ripiegamento, dove le pieghe e le faglie tendono al ribaltamento verso 
ponente, in evidente opposizione con l'andamento generale dell'Appennino.

Dallo studio particolare di ogni singola zona presa in esame, si possono trarre le seguenti conclusioni. Dopo una fase orogenetica post-liassica e pre-cretacea, con formazione di pieghe singolari nei sedimenti triassico-liassico, si ha il parziale sollevamento eocenico dei Lepini, dei Simbruini, dei Marsicani. Fanno eccezione una parte della valle Latina, la zona a Sud del Fucino, una parte del Sirente. $I l$ sollevamento viene originato da forze aventi direzioni sub-verticali di diversa intensità. Si ha perciò la formazione di grandi zolle spinte ad altezze varie $e$ di conseguenza un adeguato numero di fratture con scorrimento. Il mare eocenico copre parzialmente la valle Latina a guisa di golfo, ai lati del quale stanno le scogliere dei Lepini e dei Simbruin. Nella Marsica il mare invade una parte della valle Roveto. Dopo un periodo di calma orogenetica nel Miocene medio, si ha un movimento negativo delle zolle che si sommergono bruscamente. Almeno parte dei Simbruini restano emersi, ma anche questi gradualmente si abbassino e vengono sommersi alla fine del periodo. Qui si ha una trasgressione, mentre nei precedenti i depositi calcarei miocenici sono subparalleli a quelli cretacei.

Al principio del Pliocene si verifica il parziale sollevamento dei Lepini, dei Simbruini, dei Marsicani, del Morrone, complicato però da forze tangenziali che producono pieghe, faglie, ed il ribaltamento di molte di queste ultime.

Nel Quaternario antico si ha ripresa generale del sollevamento, compresa la zona costiera a levante del Morrone, esclusa però la zona Pontina. I depositi del Pliocene, nel versante adriatico, vengono portati a piu di $300 \mathrm{~m}$ di quota. La fase orogenetica, dopo qualche sosta, continua con sollevamenti ed abbassamenti parziali, come ad esempio l'assestamento della valle Roveto, della valle dell'Aterno, ed il sollevamento della pianura Pontina.

La nuova teoria del Prof. Accordi, di pubblicazione recente, in un certo senso riconsidera la teoria del carreggiamento verso Nord delle masse litoidi, teoria che era stata già ammessa per i Simbruini e gli Ausoni dal Franchi e dal Grzybosky, e che viene estesa a tutto il complesso laziale-abruzzese.

Questo movimento traslativo viene attribuito all'emersione, all'inizio del Pliocene, di una (presunta) dorsale tirrenica che, partendo dal Golfo di Squillace, interessava la parte settentrionale della Sardegna e vergeva infine verso oriente fino all'isola d'Elba. Ai primi movimenti 
di emersione si $\dot{e}$ avuto il riversamento del pacco delle "argille scagliose ", giacenti in un profondo bacino intermedio, sia sulla Sicilia e la Calabria, sia sull'Appennino centro meridionale (Tortoniano). Quando il fenomeno si esaspera, le masse calcaree, con la loro copertura detritica, sono interessate da un vero e proprio movimento traslativo che, da posizioni primitive nell'area tirrenica, grosso modo vicino alle isole Pontine, con movimento dell'ordine di alcune decine di chilometri, per le strutture occidentali, si sono spostate nell'area attuale con andamento circa $\mathrm{N}$-NE.

A questo movimento fece seguito un vero e proprio scivolamento e sbandamento ai lati, sempre verso $\mathrm{NE}$, con assestamento e collassi recenti.

Afrontiamo ora lo studio stratigrafico-tettonico delle singole strutture che costituiscono il complesso laziale-abruzzese.

Procedendo dal Tirreno verso l'Adriatico, la prima struttura che incontriamo, il Monte Circeo, caratterizza e conferma la teoria del carreggiamento. L'interpretazione di questo promontorio, considerato come una scaglia tettonica, si basa più sui dati della geofisica che non su quelli del terreno.

Molto più semplice è la stratigrafia dei Monti Lepini, Ausoni e Aurunci.

Esaminati i dati di campagna, è evidente il completo sovrascorrimento dei Lepini Nord-orientali sui Lepini Sud-occidentali. I primi hanno scavalcato $\mathrm{i}$ secondi portandosi da una posizione più arretrata alla posizione attuale.

Il gruppo degli Ausoni è separato dai Lepini dalla piana di Amaseno, interessata dal vulcanismo della Valle del Sacco, e in più punti, da lembi di marne ed argille appartenenti alle "Argille Caotiche ", e dal piccolo blocco di Priverno. Gli Ausoni nei loro caratteri generali presentano una disposizione a sinclinaloide; fa eccezione il monte Siserno, il quale è la lunga fronte di sovrascorrimento dei LepiniAusoni verso NE ed è una dorsale con una piega coricata anche sul lato opposto, cioè verso WSW. La sua base era visibile sotto una cava: sotto i calcari si vedevano le argille caotiche mioceniche intensamente laminate e tettonizzate.

Pì̀ a SE il gruppo degli Aurunci appare nel complesso fratturato da faglie, per lo più verticali. Vi sono anche moltissime strie orizzontali, con movimenti ora verso $\mathrm{N}$ ora verso $\mathrm{NE}$; anch'essi sono testimoni di movimenti traslativi, la cui importanza è notevole ed è messa chialamente in luce dall'ampia fronte verso la valle Latina dove vi è un 
arco con faglie inverse, piani di sovrascorrimento e lembi avanzati che si vedono oggi isolati, galleggianti sui materiali argillosi della valle del Sacco, come il Monte Leuco. Un'altra prova del movimento verso NE è data dai colli conglomeratici che poggiano contro il rilievo ausonio: questi conglomerati sono a diretto contatto col rilievo calcareo mesozoico, ma contengono solo rari ciotoli di queste rocce, e sono formati in prevalenza da arenarie, marne, calcari marnosi. Data la loro composizione litologica, è evidente che i conglomerati si sono deposti indipendentemente dal massiccio calcareo, che pure ora li sovrasta; recentemente si sono scoperte una quantità di ciotoli con magnifiche striature tettoniche rivolte in media verso NE. Queste strie possono essere dovute solo ad una spinta del massiccio calcareo contro i colli conglomeratici. Il movimento traslativo di queste strutture può considerarsi nell'ordine dei $50 \mathrm{~km}$.

Nello schema tettonico dei Simbruini, Ernici sono compresi, oltre al blocco centrale, il complesso che da Arpino si spinge fino a Cassino e, a Nord, i monti Prenestini e i rilievi di Saracinesco fino alla piana di Carsoli. Questa struttura, come i monti Lepini, ¿̀ caratteristica di facies di piattaforma. La parte settentrionale orientale dei Simbruini presenta delle difficoltà interpretative, per il gran numero di faglie che nascondono la situazione originaria. In ogni modo alcuni piani di sovrascorrimento, inclinati verso $\mathrm{NE}$, fanno pensare che le masse mesozoiche sovrastino tettonicamente la serie miocenica della marnosa arenacea. Verso $\mathrm{W}$, sui monti di Saracinesco, due scaglie di calcare miocenico sono sovrascorse sui pacchi argillosi del miocene superiore.

Anche il complesso dei Simbruini, come quello dei Lepini, ha subito un. forte movimento traslativo, che lo ha portato da posizioni tirreniche verso l'Adriatico. Ricordiamo ancora una volta la perforazione del pozzo Trevi da parte dell's gip mineraria, che ha confermato la disposizione anomala dei terreni, spiegabile esaurientemente solo con la teoria traslativa.

Con il termine "Strutture della Marsica Sud-Occidentale ", si prendono in considerazione i rilievi che sorgono tra la valle Roveto e la valle del Sangro.

Il versante orientale della val Roveto, o valle del Liri, è formato da un enorme reggipoggio di dolomie e calcari mesozoici che nel tratto di Norea e Balsorano è interessato da un sistema di faghe dirette, mascherate spesso da enormi conoidi di deiezione. Più a Nord, la parete a reggipoggio va gradualmente diminuendo, assumendo valori modesti 1 ra Capistrello e Tagliacozzo. Del tutto diverso è l'assetto tettonico 
nella parte meridionale della valle del Liri: abbiamo ancora faglie dirette, le pareti calcaree non sono più a reggipoggio ma sono inclinate verso valle tanto che, presso la frazione Forcella, è possibile vedere $i$ sedimenti clastici del miocene superiore, disposti in serie regolare sul miocene calcareo e sul creta.

La lunga struttura al cui centro vi è la Serralunga è una monoclinale che pende verso l'Adriatico. In corrispondenza di Villavallelunga, dà una piega frontale piuttosto ridotta che si sovrappone alla marnosa arenacea. Verso il Fucino questa piega sparisce, per la presenza di una piccola faglia, sepolta dalle alluvioni quaternarie. In complesso questa struttura mostra una caratteristica nuova nelle strutture precedenti. La catena non è stata traslata parallelamente a se stessa, ma ha subito uno spostamento differenziato, con un punto che ha agito da fulcro e non ha subito traslazione.

Consideriamo ora la struttura che interessa Trasacco, Ortona dei Marsi e, scendendo verso Sud, passa per Pescasseroli piegando verso Est, fino ai rilievi di monte Amaro e M. Petroso. Il limite anteriore, di questa struttura è dato dalle linee tettoniche delle valli di Giovenco e del Sangro. Altri due disturbi tettonici interessano la linea di Giovenco e tutti e tre confluiscono verso la valle del Sangro. Anche la valle del Sangro è caratterizzata, fra le Fosse di S. Paolo e Villetta Barrea, da due linee tettoniche: una faglia diretta, che borda il monte Marsicano, e la linea di sovrascorrimento che da La Pelusare si dirige verso Civitella Alfedena, costeggiando a Nord il Monte Amaro. Questa ultima è molto importante perchè è legata alla traslazione del gruppo del monte Amaro e perchè il movimento avvenne verso $\mathrm{N}$ anziché verso NE. Questa linea continua, anche se non troppo evidente, passando alle spalle di Pescasseroli e collegandosi a quelle faglie che determinarono spostamenti verso NE.

La Marsica Norlorientale è una grossa zolla delimitata ad W della linea Giovenco-Alto Sangro, che si spinge fino ai rilievi che nel foglio Agnone si saldano con la parte meridionale della struttura del Morrone. La struttura, nel complesso, appare smembrata da faglie disposte nel senso appenninico.

Il gruppo del Velino è separato a Sud da una faglia diretta che da Celano, passando a monte di Massa d'Albe, va nella depressione Cartore-Corvaro; alcune faglie dirette vicarianti ne ribassano le propaggini Sudoccidentali. Per il resto presenta caratteristiche non dissimili alle strutture finora esaminate: pendenza generale verso l'Adriatico, linee di disturbo sempre nel senso appenninico, ancora depositi 
di bauxite. Verso Nord, abbiamo un passaggio a facies che si collegano con quelle del Gran Sassso.

La fronte è interessata, fra Bagnogrande e Ovindoli, da un disturbo con parziale sovrascorrimento del Cretaceo sulle argille mioceniche. Anche più a Nord, il complesso dei monti Giano e Nuria sono sovrascorsi sulla marnosa arenacea.

La parte occidentale del gruppo del Sirente pende generalmente verso Ovest, tanto che fra Rocea di Mezzo e Ovindoli sembra veramente che s'immerga sotto il Velino. La parte orientale invece ì formata da calcari non più mesozoici, e si collegia con le strutture dei monti Picca e Cappucciata, attraverso lievi disturbi tettonici. Più a Sud la conca di Sulmona la separa dal gruppo del Morrone. Fra Sagittario e Capestrano, nel bordo occidentale della conca, i calcari finemente stratificati della facies di transizione presentano pieghe ribaltate ad Est.

Il gruppo del Morrone è limitato, alle spalle, dalla conca di Sulmona, alla fronte, l'allineamento Picca Cappucciata costituisce l'ultimo rilievo prima delle colline della piana adriatica, mentre il monte Morrone è separato dalla Maiella dalla valle di Caramanico.

Interessante sotto l'aspetto tettonico il grande scorrimento frontale dato da una piega-faglia con inclinazione di una trentina di gradi. In corrispondenza di Colle d'Oro s'imposta una vicariante ancora più vicina all'orizzonte. La parte settentrionale, oltre la fronte, è caratterizzata dalla presenza di due scaglie enucleate di La Queglia e di Colle Cantalupo, che possono considerarsi come lame rigide espulse dalla profondità a causa della forte compressione.

Più a Sud, nella zona di Roceacaramanico, il motivo delle scaglie enucleate è sostituito da alcune lunghe pieghe accavallate e ribaltate verso NE, bordate dalle argille del Miocene superiore e del Pliocene.

Anche in questa struttura il sovrascorrimento è confermato da un sondaggio nella gola incisa dal fiume Pescara, a monte della fronte. Infatti, il sondaggio ha incontrato sotto i calcari la stessa formazione argillosa antistante alla fronte. Il movimento minimo dell'intera struttura darebbe valori intorno ai $4 \mathrm{~km}$.

La Maiella è una brachianticlinale, con asse orientato da Nord a Sud, rotto solo sul fianco occidentale, mentre $i$ bordi settentrionale e orientale hanno pendenze che fanno immergere le formazioni sotto la coltre detritica. Benché oggetto di grande studio, la Maiella presenta caratteri strutturali e stratigrafici ancora in discussione.

Ai bordi della Maiella, nella zona Peligna Sud orientale, com- 
paiono formazioni inglobate parzialmente nel complesso delle argille scagliose; questo complesso, incontrato anche nella piana di Vasto, da perforazioni di pozzi, comprende, oltre che i gessi e i conglomerati, anche le cosiddette "Arenarie di Ronca " rosse o brune per alterazione, riccamente fossilifere e, sul tetto della formazione miopliocenica, delle brecce di calcare di esiguo spessore.

Dobbiamo ricordare ancora due serie: quella di Taranta, con argille scagliose rosse brune, porcellanite rosa (roccia silicea argillosa), e frammenti vetrosi vulcanici, selce nera, brecce calcaree e letti carboniosi; e la serie della Torretta, emergente dalle argille scagliose e formata principalmente da calcari marnosi a globigerine, alternati a brecciole e tufi vuleanici.

La messa in posto di questi materiali alloctoni sembra dovuta al sollevamento della Brachianticlinale, che ha fatto affossare il pacco dei sedimenti compresi fra la Maiella e il Gargano, con il conseguente richiamo dei materiali alloctoni. Sopra questi si sono sedimentate le sabbie del pliocene superiore. Per quanto riguarda la fronte della Maiella, possiamo dividerla in due parti: una meridionale, in cui mancano contatti tettonici, e una settentrionale, con contatti anormali con le argille scagliose; questo starebbe a dimostrare un movimento parziale della parte settentrionale, mentre la meridionale avrebbe agito da perno. Nel complesso la Maiella, che si considera ancora rarlicata, ebbe uno spostamento verso Est di almeno $3 \mathrm{~km}$.

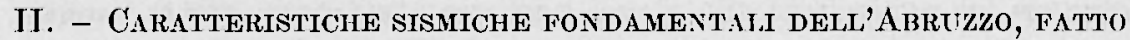 RIFERIMENTO AL PERIODO 1900-1970.}

Allo scopo di precisare meglio le caratteristiche dinamiche dell'Abruzzo, ei siamo preoccupati di aggiungere, alle determinazioni condotte con metodi basati su registrazioni sismografiche (forzatamente, in numero limitato), i dati relativi ai rilievi macrosismici di gran parte dei terremoti sentiti nel primo settantennio del secolo.

Tracciate le isosiste di questi terremoti, abbiamo determinate le relative coordinate geografiche epicentrali. Ci siamo quindi preoccupati di calcolare le corrispondenti profondità ipocentrali, le energie sviluppate e le magnitudo dei singoli terremoti.

Ise profondita $h$ sono state calcolate con la formula generale

$$
h=\frac{s_{n}}{\sqrt{10^{\frac{I_{0}-I_{n}}{3}}-1}}
$$


SISMICIT A IN RFIAAZIONE ALIA TETTONICA F ACCUNUI.O DEILF TENSIONI 323

dove $I_{0}$ è l'intensitì (della scala Mercalli) osservata all'epicentro, $I_{n}$ le intensità delle isosiste successive e $s_{n}$ il relativo raggio limite. I'energia è stata ottenuta con la formula

$$
\lg E=9.5+3.2 \cdot \lg h+1.1 I_{0}
$$

dove i logaritmi sono decimali, mentre per la magnitudo ci siamo valsi della formula

$$
M=0.6 I_{o}+\lg h+0.4
$$

Le Tabelle che seguono contengrono i risultati ottenuti. 


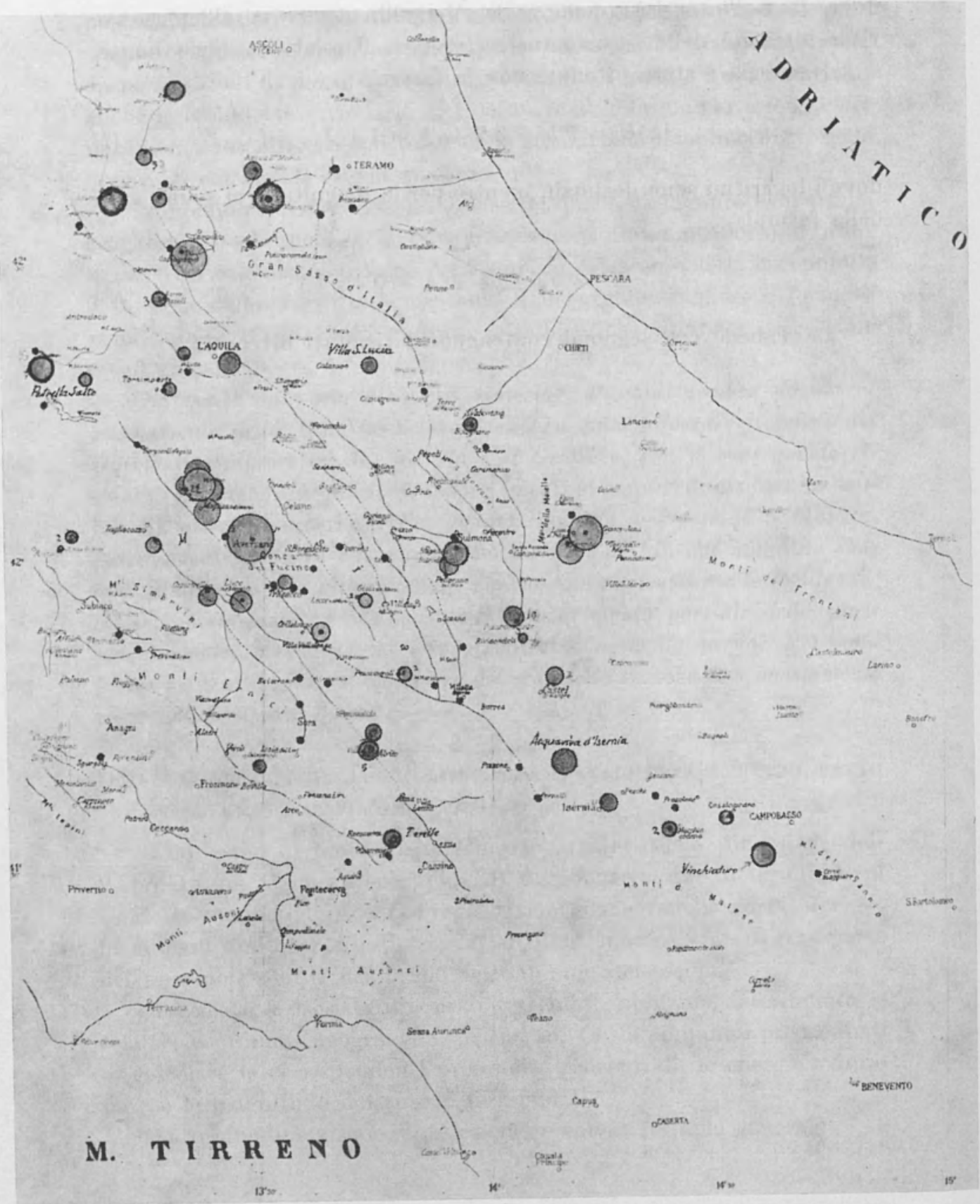

Fig. 1 - Distribuzione geografica degli epicentri (la cifra a fanco indica il numero
delle principali scosse arvertite nella localita), le linee rappresentano le faglie regionali. 


\begin{tabular}{|c|c|c|c|c|c|c|c|}
\hline No & Data & Località & $\begin{array}{c}\text { Coord. Ep. } \\
\varphi \quad \lambda\end{array}$ & $I_{o}$ & $\begin{array}{c}h \\
\mathrm{~km}\end{array}$ & $M$ & $\begin{array}{c}E \\
\text { ergs }\end{array}$ \\
\hline 1 & $29-I-1900$ & Alanno & $42^{\circ} 05^{\prime} \mathrm{N}$ & VI & 4 & 4.6 & $10^{18}$ \\
\hline 2 & $\begin{array}{c}21-I V " \\
08^{\mathrm{h}} 45^{\mathrm{m}}\end{array}$ & L'Aquila & $\begin{array}{l}42^{\circ} 21^{\prime}, 4 \mathrm{~N} \\
13^{\circ} 25^{\prime} \mathrm{E}\end{array}$ & $1 V$ & 6 & 3.6 & $3.2 \times 10^{16}$ \\
\hline 3 & $\begin{array}{c}10 \text {-VIII- } \\
05^{\mathrm{h}} 30^{\mathrm{m}}\end{array}$ & Terano & - & V.VI & 5 & 4.4 & $8 \times 10^{17}$ \\
\hline 4 & $\begin{array}{c}10-\text { VIII - " } \\
22^{\mathrm{h}} 30^{\mathrm{m}}\end{array}$ & Accumoli & - & IV $-\mathrm{V}$ & 5 & 3.5 & $10^{16}$ \\
\hline 5 & $\begin{array}{l}2 . \mathrm{IX}- \\
10^{\mathrm{h}}+\end{array}$ & $\begin{array}{l}\text { Borgo } \\
\text { Velino }\end{array}$ & - & V & 5 & 4.0 & $2 \times 10^{17}$ \\
\hline 6 & $\begin{array}{c}31-V I \bar{I}-1901 \\
11^{\mathrm{h}} 40^{\mathrm{m}}\end{array}$ & Alvito & - & VIII & 5 & 6.0 & $8 \times 10^{20}$ \\
\hline 7 & $\begin{array}{c}\text { 31-VII- " } \\
17^{\mathrm{h}} \pm\end{array}$ & $\begin{array}{l}\text { Villetta } \\
\text { Barrea - }\end{array}$ & 一 & IV & 5 & 3.5 & $10^{18}$ \\
\hline 8 & $\begin{array}{c}\text { 1-VIII- } \\
17^{\mathrm{h}} \pm\end{array}$ & Pescosolido & - & IV & 5 & 3.5 & $10^{16}$ \\
\hline 9 & $\begin{array}{l}\text { 14-VIII. " } \\
02^{\mathrm{h}} 15^{\mathrm{m}}\end{array}$ & Opi & - & $\mathrm{V} \cdot \mathrm{VI}$ & 5 & 4.4 & $8 \times 10^{17}$ \\
\hline 10 & $\begin{array}{c}15-X-X^{\prime \prime} \\
14^{\mathrm{h}} 55^{\mathrm{m}}\end{array}$ & S. Valentino & $\begin{array}{l}42^{\circ} 14^{\prime} \mathrm{N} \\
13^{\circ} 59^{\prime} \mathrm{E}\end{array}$ & V1 & 8 & 4.9 & $10^{19}$ \\
\hline II & $\begin{array}{c}21-X_{-} " \\
03^{\mathrm{h}} 58^{\mathrm{m}}\end{array}$ & L'Aquila & $\begin{array}{l}42^{\circ} 21^{\prime} .4 \mathrm{~N} \\
13^{\circ} 25^{\prime} \mathrm{E}\end{array}$ & V & 6 & 4.8 & $10^{18}$ \\
\hline 12 & $\begin{array}{c}25-X- \\
17^{\mathrm{h}} 15^{\mathrm{m}}\end{array}$ & $"$ & 11 & IV & 6 & 3.6 & $3.2 \times 10^{16}$ \\
\hline 13 & $\begin{array}{c}26-X- \\
09^{\mathrm{h}} 15^{\mathrm{m}}\end{array}$ & $"$ & $"$ " & IV & 6 & 3.6 & $3.2 \times \mathrm{IO}^{16}$ \\
\hline 14 & $\begin{array}{c}15-\mathrm{XI}-" \\
04^{\mathrm{h}} 58^{\mathrm{m}}\end{array}$ & $"$ & $" \quad "$ & V & 6 & 4.2 & $10^{18}$ \\
\hline 15 & $\begin{array}{c}17-\mathrm{XI}-" \\
08^{\mathrm{n}} 25^{\mathrm{m}}\end{array}$ & $"$ & $"$ & IV & 6 & 3.6 & $3.2 \times 10^{16}$ \\
\hline 16 & $\begin{array}{c}9-I-1902 \\
19^{\mathrm{h}} 25^{\mathrm{m}}\end{array}$ & $"$ & $" n$ & $1 \mathrm{~V}$ & 6 & 3.6 & $3.2 \times 10^{16}$ \\
\hline 17 & $\begin{array}{l}\text { 14-II- " } \\
21^{\mathrm{h}} 09^{\mathrm{m}}\end{array}$ & $-4+2+2$ & $" \quad "$ & $1 \mathrm{~V}$ & 6 & 3.6 & $3.2 \times 10^{18}$ \\
\hline 18 & $\begin{array}{r}31-V_{-} \prime \prime \\
08^{\mathrm{h}} 30^{\mathrm{m}}\end{array}$ & $\begin{array}{l}\text { Villetta } \\
\text { Barrea }\end{array}$ & -- & IV & - & - & - \\
\hline 19 & $\begin{array}{l}\text { 31-VII- } \\
15^{\mathrm{h}} 23^{\mathrm{m}}\end{array}$ & L'A quila & $\begin{array}{l}42^{\circ} 21^{\prime} .4 \mathrm{~N} \\
13^{\circ} 24^{\prime} .7 \mathrm{E}\end{array}$ & II I & 6 & 3.0 & $2.5 \times 10^{15}$ \\
\hline 20 & $\begin{array}{r}5-I_{X}-" \prime \\
22^{\mathrm{n}} 30^{\mathrm{m}}\end{array}$ & $"$ & " & IV & 6 & 3.6 & $3.2 \times 10^{16}$ \\
\hline 21 & $\begin{array}{r}30 . \mathrm{IX}^{-1} \\
08^{\mathrm{h}} 35^{\mathrm{m}}\end{array}$ & $"$ & $"$ & $1 \mathrm{~V}$ & 6 & 3.6 & $3.2 \times 10^{16}$ \\
\hline 22 & $\begin{array}{c}\text { 23-XII- } \\
05^{\mathrm{h}} \pm\end{array}$ & " & $" \#$ & $1 \mathrm{~V}$ & 6 & 3.6 & $3.2 \times 10^{16}$ \\
\hline \multicolumn{8}{|c|}{$\begin{array}{l}\text { Periodo sismico L'Aquila. } \\
o 1901 E_{1901}=2.1 \times 10^{18} \mathrm{ergs} \\
o 1902 E_{1902}=0.163 \times 10^{18} \mathrm{ergs} \\
o 1903 E_{1903}=3.4 \times 10^{18} \mathrm{ergs} \\
\text { rgia Totale } E_{1901 \cdot 1902-1903}=3.4 \times 10\end{array}$} \\
\hline
\end{tabular}




\begin{tabular}{|c|c|c|c|c|c|c|c|c|}
\hline No & Data & Jocalitì & $\begin{array}{r}\text { Coor } \\
\varphi\end{array}$ & $\begin{array}{l}\text { rd. Ep. } \\
\lambda\end{array}$ & $I_{0}$ & $\begin{array}{c}h \\
k m\end{array}$ & $M$ & $\begin{array}{c}E \\
\text { ergs }\end{array}$ \\
\hline 23 & $\begin{array}{c}12-I 1-190.3 \\
14^{1}\left(05^{\mathrm{m}}\right.\end{array}$ & ['Aquila & $"$ & $"$ & $\mathrm{~V}$ & 6 & 4.2 & $10^{18}$ \\
\hline 24 & $\begin{array}{c}14.11-" \prime \\
22^{\mathrm{h}} 18^{\mathrm{m}}\end{array}$ & $”$ & " & $"$ & IV & 6 & 4.2 & $3.2 \times 10^{16}$ \\
\hline 25 & $\begin{array}{c}7.1 \mathrm{II}-" \\
05^{\mathrm{h}} \pm\end{array}$ & $"$ & $"$ & $"$ & IV & 6 & 4.2 & $3.2 \times 10^{16}$ \\
\hline 26 & $\begin{array}{l}8-111^{ \pm \prime} " \\
18^{\mathrm{h} 2} 0^{\mathrm{m}}\end{array}$ & $"$ & $"$ & ” & III & 6 & 3.0 & $2.5 \times 10^{15}$ \\
\hline 27 & $\begin{array}{c}9-\mathrm{IIJ}-"{ }^{\prime \prime} \\
05^{\mathrm{h}} 12^{\mathrm{m}}\end{array}$ & $"$ & $n$ & ” & IV & 6 & 4.2 & $3.2 \times 10^{16}$ \\
\hline 28 & $\begin{array}{c}2+-I I-1904 \\
16^{\mathrm{n}} 53^{\mathrm{m}} 21^{\mathrm{s}}\end{array}$ & $\begin{array}{l}\text { Rosciolo } \\
\text { (Velino) }\end{array}$ & $\begin{array}{l}42^{\circ} 0 \\
13^{\circ} 2\end{array}$ & $\begin{array}{ll}07^{\prime} & \mathrm{N} \\
20^{\prime} .5 \mathrm{E}\end{array}$ & $\mathrm{IX}$ & 6 & 6.6 & $10^{22}$ \\
\hline 29 & $\begin{array}{l}25-\mathrm{II}-" \prime \\
0 \mathrm{I}^{\mathrm{n}} 29^{\mathrm{m}}\end{array}$ & Velino & ” & $"$ & VII & 7 & 5.4 & $10^{20}$ \\
\hline $29 \mathrm{a}$ & $\begin{array}{c}25-\mathrm{II}-" \prime \\
03^{\text {n }} 04^{\mathrm{m}}\end{array}$ & $n$ & $"$ & $"$ & V1I & 4 & 5.2 & $10^{18}$ \\
\hline 30 & $\begin{array}{c}25-\text { II - " } \\
06^{11} \pm\end{array}$ & $”$ & $"$ & n & VII & 9 & 5.6 & $1.6 \times 10^{20}$ \\
\hline 31 & $25 \cdot 11-" 1$ & $"$ & $n$ & $n$ & V1I & I0 & 5.6 & $2.5 \times 10^{20}$ \\
\hline 32 & $\begin{array}{l}25-11-\quad " \\
20^{\mathrm{h}} 16^{\mathrm{m}}\end{array}$ & Y. & $"$ & $"$ & IV & 7 & 5.4 & $5.0 \times 10^{16}$ \\
\hline 33 & 1-III - " & $\begin{array}{l}\text { Marsica } \\
\text { (Velino) }\end{array}$ & $"$ & IO & VI & 10 & 5.0 & $2.0 \times 10^{18}$ \\
\hline 34 & $\begin{array}{c}\text { I.III- } \\
05^{\mathrm{h}} \pm\end{array}$ & $\begin{array}{l}\text { Borgocolle- } \\
\text { fegato - } \\
\text { I'Aquila } \\
\text { Scureola } \\
\text { M. }\end{array}$ & $\begin{array}{l}42^{\circ} \mathrm{I} \\
13^{\circ} 1\end{array}$ & $\begin{array}{ll}I^{\prime} & N \\
15^{\prime} & E\end{array}$ & VI & 14 & 5.1 & $6.3 \times 10^{18}$ \\
\hline 35 & $\begin{array}{l}3 \text {-III- } \\
19^{\mathrm{h}} 56^{\mathrm{m}}\end{array}$ & $\begin{array}{l}\text { Marsica - } \\
\text { Velino }\end{array}$ & & D & VII & 15 & 5.8 & $10^{21}$ \\
\hline 36 & 10-III- " & Velino & " & $"$ & VIII & 8 & 6.1 & $1.6 \times 10^{21}$ \\
\hline 37 & $\begin{array}{l}11-\text { III- } \\
14^{\mathrm{n}} 25^{\mathrm{m}}\end{array}$ & $\begin{array}{c}\text { Magliano dei } \\
\text { Marsi - } \\
\text { Velino }\end{array}$ & $"$ & " & VII & 7 & 5.4 & $10^{20}$ \\
\hline 38 & $\begin{array}{c}20 . \text { III- } " ~ \\
06^{\mathrm{n}} 50^{\mathrm{m}} \mathrm{m}\end{array}$ & $\begin{array}{l}\text { Magliano dei } \\
\text { IIarsi - } \\
\text { Velino }\end{array}$ & $n$ & $n$ & V11 & 7 & 5.4 & $10^{20}$ \\
\hline 39 & $20-$ III- " & $\begin{array}{l}\text { Scurcola - } \\
\text { Velino }\end{array}$ & $"$ & $"$ & VII & 9 & 5.6 & $10^{20}$ \\
\hline 40) & $\begin{array}{l}25-\text { III- } " ~ \\
22^{\mathrm{h}} 58^{\mathrm{m}}\end{array}$ & $\begin{array}{l}\text { Magrliano dei } \\
\text { Marsi - } \\
\text { Velino }\end{array}$ & $"$ & $"$ & VII & 7 & 5.4 & $10^{20}$ \\
\hline 41 & $\begin{array}{c}28-111-{ }^{\prime \prime} \\
23^{\mathrm{h}} 26^{\mathrm{m}}\end{array}$ & $\begin{array}{l}\text { Scanzano - } \\
\text { Velino }\end{array}$ & $"$ & $"$ & V & 11 & 4.4 & $2 \times 10^{1 \mathrm{~B}}$ \\
\hline 42 & $\begin{array}{c}29-\text { III - } \\
05^{\mathrm{n}} 30^{\mathrm{m}}\end{array}$ & $\begin{array}{l}\text { Scanzano - } \\
\text { Velino }\end{array}$ & $”$ & " & V & 11 & 4.4 & $2 \times 10^{18}$ \\
\hline 43 & $\begin{array}{l}29-\text { IlI- } \\
09^{\mathrm{n}} 30^{\mathrm{m}}\end{array}$ & $\begin{array}{c}\text { Magliano dej } \\
\text { Marsi - } \\
\text { Velino }\end{array}$ & $"$ & ") & VIII & 4 & 5.8 & $1.6 \times 10^{20}$ \\
\hline
\end{tabular}




\begin{tabular}{|c|c|c|c|c|c|c|c|}
\hline No & Data & Jocalità & $\begin{array}{l}\text { Coord. Ep. } \\
\qquad \lambda\end{array}$ & $I_{o}$ & $\begin{array}{c}h \\
k m\end{array}$ & $M$ & $\begin{array}{c}E \\
\text { ergs }\end{array}$ \\
\hline 44 & $\begin{array}{c}\text { 7-IV-1904 } \\
20^{\mathrm{h}}+\end{array}$ & $\begin{array}{c}\text { Magliano dei } \\
\text { Îarsi - }\end{array}$ & $\begin{array}{l}42^{\circ} 13^{\prime} \\
13^{\circ} 15^{\prime}\end{array}$ & VII & 7 & 5.4 & $10^{20}$ \\
\hline 45 & $\begin{array}{c}7-1 V-{ }^{\prime \prime} \\
20^{\mathrm{h}} 55^{\mathrm{m}}\end{array}$ & $\begin{array}{c}\text { Magliano dei } \\
\text { Marsi - } \\
\text { Velin }\end{array}$ & $" \quad "$ & V & 7 & 4.2 & $5 \times 10^{17}$ \\
\hline 46 & $\begin{array}{l}\text { 13.IV. } \\
06^{\mathrm{n}} 57^{\mathrm{m}}\end{array}$ & Scanzano & $" n$ & VII & 11 & 5.6 & $3.2 \times 10^{20}$ \\
\hline 47 & $\begin{array}{l}20-1 \mathrm{~V}-n \\
23^{\mathrm{n}} 55^{\mathrm{m}}\end{array}$ & $\begin{array}{l}\text {-Velino } \\
\text { Velino - } \\
\text { Scanzano }\end{array}$ & " " & VII & 11 & 5.6 & $3.2 \times 10^{20}$ \\
\hline 48 & $\begin{array}{c}\text { 14.V. } \\
05^{\mathrm{h}}+\end{array}$ & (Velino) & $" n$ & IV & 10 & 3.8 & $1.3 \times 10^{17}$ \\
\hline 49 & $\begin{array}{l}\text { 8-VI-" } \\
14^{\mathrm{h}} 30^{\mathrm{m}}+=\end{array}$ & $\begin{array}{l}\text { Velino } \\
\text { Avezzano - } \\
\text { Cappelle }\end{array}$ & " " & VI & 9 & 5.0 & $1.3 \times 10^{19}$ \\
\hline 50 & $\begin{array}{l}22-V I-n \\
16^{\mathrm{h}} 30^{\mathrm{m}}\end{array}$ & $\begin{array}{l}\text { Velino - } \\
\text { Scanzano } \\
\text { (IV) }\end{array}$ & $" n$ & VI & 7 & 4.8 & $6.3 \times 10^{18}$ \\
\hline 51 & $\begin{array}{l}\text { 5.VII- - } \\
02^{\mathrm{h}} 40^{\mathrm{m}}\end{array}$ & L'Aquila & - & V & 6 & 4.2 & $10^{18}$ \\
\hline 52 & $\begin{array}{c}2-\mathrm{XI}-" ~ \\
08^{\mathrm{n}} 15^{\mathrm{m}}\end{array}$ & $\begin{array}{l}\text { Manoppello- } \\
\text { Gioia dei } \\
\text { Marsi }\end{array}$ & - & I $V$ & 5 & 3.5 & $1.6 \times 10^{18}$ \\
\hline 53 & $\begin{array}{c}2 \cdot \mathrm{XI}-\| " \prime \\
16^{\mathrm{h}} 32^{\mathrm{m}}\end{array}$ & $\begin{array}{l}\text { Gioia dei MI. } \\
\text { Scamno }\end{array}$ & $\begin{array}{l}41^{\circ} 56^{\prime}, 6 \frac{\mathrm{N}}{13^{\circ} 42^{\prime}} \mathrm{F}\end{array}$ & VII & 10 & 5.6 & $2.5 \times 10^{20}$ \\
\hline 54 & $\begin{array}{c}\text { 30-XII- " } \\
23^{\text {n }} \pm\end{array}$ & Scanzano & & $V$ & 5 & 4.1 & $2 \times 10^{17}$ \\
\hline 55 & $\begin{array}{c}16-I I-190.5 \\
04^{\mathrm{h}}+\end{array}$ & $\begin{array}{l}\text { Tornimpar- } \\
\text { te }\end{array}$ & - & $\mathrm{V}$ & 5 & 4.1 & $2 \times 10^{17}$ \\
\hline 56 & $\begin{array}{c}25-V I I I-" ~ \\
\left.2\right|^{4} 4 I^{m}\end{array}$ & Sulmona & $\begin{array}{l}42^{\circ} 02^{\prime} .7 \mathrm{~N} \\
13^{\circ} 55^{\prime} .7 \mathrm{E}\end{array}$ & VII & 15 & 5.8 & I $0^{21}$ \\
\hline 57 & $\begin{array}{c}27-V I 11-" ~ \\
10^{\mathrm{h}} 50^{\mathrm{m}}\end{array}$ & $"$ & $"$ & $\mathrm{~V}$ & 10 & 4.4 & $2 \times 10^{18}$ \\
\hline 58 & $\begin{array}{c}5-I I-1906 \\
\left.16^{\mathrm{h}}\right] 0^{\mathrm{m}}\end{array}$ & Montereale & - & VI & 5 & 4.8 & $5 \times 10^{18}$ \\
\hline 59 & $\begin{array}{l}\text { 1. VII. } \\
01^{\mathrm{n}} 50^{\mathrm{m}}\end{array}$ & Antrodoco & - & V-VI & 5 & 4.8 & $5 \times 10^{18}$ \\
\hline 60 & $\begin{array}{c}24-X-" 1 \\
03^{\mathrm{n}} 45^{\mathrm{m}}\end{array}$ & Pettorano & - & IV & 5 & 3.5 & $1.6 \times 10^{18}$ \\
\hline 61 & $\begin{array}{c}23-I-190 \mathrm{r} \\
01^{\mathrm{H}} 15^{\mathrm{m}}\end{array}$ & Teramo & -. & $V$ & 5 & 4.1 & $2 \times 10^{17}$ \\
\hline 62 & $\begin{array}{c}20-111-\quad " \\
14^{\mathrm{h}} 30^{\mathrm{m}} \mathrm{m}\end{array}$ & $\begin{array}{c}\text { Casalcipra- } \\
\text { no }\end{array}$ & $\begin{array}{l}41^{\circ} 34^{\prime} .6 \mathrm{~N} \\
14^{\circ} 31^{\prime} .3 \mathrm{E}\end{array}$ & VI & 8 & 4.9 & $10^{19}$ \\
\hline 63 & $\begin{array}{c}\text { 10-VII- " } \\
04^{\mathrm{h}} \pm\end{array}$ & $\begin{array}{l}\text { Pesco- } \\
\text { costanzo }\end{array}$ & $\begin{array}{l}41^{\circ} 55^{\prime} \mathrm{N} \\
14^{\circ} 04^{\prime} .5 \mathrm{E}\end{array}$ & VII & 5 & 5.3 & $3.2 \times 10^{19}$ \\
\hline 64 & $\begin{array}{c}\text { 18-VIII " } \\
13^{\mathrm{h}}+\end{array}$ & " & $" n$ & IV & 5 & 3.5 & $1.6 \times 10^{18}$ \\
\hline 65 & $\begin{array}{l}19-\mathrm{VII}-" \\
13^{\mathrm{n}} 30^{\mathrm{mt}}\end{array}$ & " & $"$ " & 111 & 5 & 2.9 & $1.3 \times 10^{16}$ \\
\hline 66 & $\begin{array}{l}\text { 22-VII-" } \\
09^{\mathrm{n}} 30^{\mathrm{m}}\end{array}$ & $"$ & $" \quad "$ & V & 5 & 4.1 & $2 \times 10^{17}$ \\
\hline
\end{tabular}




\begin{tabular}{|c|c|c|c|c|c|c|c|}
\hline No & I)ata & Localitì & $\begin{array}{c}\text { Coord. Ep. } \\
\varphi \lambda\end{array}$ & $I_{0}$ & $\begin{array}{c}h \\
\mathrm{~km}\end{array}$ & $\lambda$ & $\begin{array}{c}E \\
\text { Ergs }\end{array}$ \\
\hline 67 & $\begin{array}{c}\text { 12-VIII-1907 } \\
12^{\mathrm{h}} 20^{\mathrm{m}}\end{array}$ & Avezzano & - & IV & 5 & 3.5 & $1.6 \times 10^{16}$ \\
\hline 68 & $\begin{array}{c}15-\mathrm{IX}-" \\
22^{11} \pm\end{array}$ & $\begin{array}{l}\text { Pesco- } \\
\text { costanzo }\end{array}$ & $\begin{array}{ll}41^{\circ} 55^{\prime} & N \\
14^{\circ} 04^{\prime} & \mathrm{E}\end{array}$ & IV & 5 & 3.5 & $1.6 \times 10^{16}$ \\
\hline 69 & $\begin{array}{c}19-\mathrm{IX}- \\
23^{\mathrm{h}} \pm\end{array}$ & " & $"$ & VII & 5 & 5.3 & $1.6 \times 10^{10}$ \\
\hline 70 & $\begin{array}{c}5-X^{-} \\
22^{\mathrm{h}} 20^{\mathrm{m}}\end{array}$ & Celano & - & V & 5 & 4.1 & $2 \times 10^{17}$ \\
\hline 71 & $\begin{array}{c}10-\mathrm{X} \\
03^{\mathrm{h}} \pm\end{array}$ & Rivisondoli & $\begin{array}{l}41^{\circ 052^{\prime}} \mathrm{N} \\
14^{\circ} 03^{\prime} .5 \mathrm{E}\end{array}$ & V & 5 & 4.1 & $2 \times 10^{17}$ \\
\hline 72 & $\begin{array}{c}10-\mathrm{X}^{-} \\
04^{\mathrm{h}} 30^{\mathrm{m}}\end{array}$ & $\begin{array}{l}\text { Pesco- } \\
\text { costanzo }\end{array}$ & $\begin{array}{l}41^{\circ} 55^{\prime} \mathrm{N} \\
14^{\circ} 04^{\prime} .5 \mathrm{E}\end{array}$ & VI & 5 & 4.7 & $2.5 \times 10^{18}$ \\
\hline 73 & $\begin{array}{c}24-\mathrm{X}- \\
20^{\mathrm{h}} 23^{\prime \prime}\end{array}$ & Rivisondoli & $\begin{array}{ll}41^{\circ} 52^{\prime} & \mathrm{N} \\
14^{\circ} 03^{\prime} .5 \mathrm{E}\end{array}$ & IV & 5 & 3.5 & $1.6 \times 10^{16}$ \\
\hline 74 & $\begin{array}{c}29 \cdot \mathrm{X}-{ }^{\mathrm{n}} \\
02^{\mathrm{h}} 30^{\mathrm{m}}\end{array}$ & $\begin{array}{l}\text { Pesco- } \\
\text { costanzo }\end{array}$ & $\begin{array}{l}41^{\circ} 55^{\prime} \quad \mathrm{N} \\
14^{\circ} 04^{\prime} .5 \mathrm{E}\end{array}$ & V & 5 & 4.1 & $2 \times 10^{17}$ \\
\hline 75 & $\begin{array}{c}22-\mathrm{XI}-" \\
13^{\mathrm{h}} 30^{\mathrm{m}}\end{array}$ & Rivisondoli & $\begin{array}{l}41^{\circ} 52^{\prime} \quad \mathrm{N} \\
14^{\circ} 03^{\prime} .5 \mathrm{E}\end{array}$ & $\mathrm{V}$ & 5 & 4.1 & $2 \times 10^{17}$ \\
\hline 76 & $\begin{array}{c}26 \cdot \mathrm{XI}- \\
19^{\mathrm{h}} 40^{\mathrm{m}}\end{array}$ & $\begin{array}{l}\text { Pesco- } \\
\quad \text { costanzo }\end{array}$ & $\begin{array}{l}41^{\circ} 55^{\prime} \quad \mathrm{N} \\
14^{\circ} 04^{\prime} .5 \mathrm{E}\end{array}$ & V & 5 & 4.1 & $2 \times 10^{17}$ \\
\hline 77 & $\begin{array}{c}22-\text { XII- } \\
07^{\mathrm{b}} \pm\end{array}$ & $"$ & $" 1$ & VII & 5 & 5.3 & $3.2 \times 10^{19}$ \\
\hline 78 & $\begin{array}{l}14-I-1908 \\
02^{\mathrm{h}} \pm\end{array}$ & Montereale & 一 & VI & 6 & 4.8 & $5 \times 10^{18}$ \\
\hline 79 & $\begin{array}{l}16-1-" \prime \\
11 \mathrm{~h}_{27} \mathrm{~m}\end{array}$ & L'Aquila & 一 & VI & 6 & 4.8 & $5 \times 10^{18}$ \\
\hline 80 & $\begin{array}{l}16-11-") \\
17^{\mathrm{h}} 50^{\mathrm{m}}\end{array}$ & Ix 1 & - & VII & 6 & 5.0 & $6 \times \mathrm{I}^{19}$ \\
\hline 81 & $\begin{array}{c}16-\mathrm{V} \cdot \quad " \prime \\
03^{\mathrm{h}} 50^{\mathrm{m}}\end{array}$ & Montereale & - & V & 5 & 4.0 & $2 \times 10^{17}$ \\
\hline 82 & $\begin{array}{c}\text { 2.VI- " } \\
13^{\text {h }} \pm\end{array}$ & Pizzoli & - & IV & 5 & 3.5 & $10^{16}$ \\
\hline 83 & $\begin{array}{l}\text { 2-VI- } \\
18^{\mathrm{h}} \pm "\end{array}$ & Barete & 一 & IV & 5 & 3.5 & $10^{16}$ \\
\hline 84 & $\begin{array}{l}\text { 5.VI- } \\
04^{\mathrm{h}} \pm\end{array}$ & $\begin{array}{l}\text { Pesco- } \\
\text { costanzo }\end{array}$ & $\begin{array}{l}41^{\circ} 53^{\prime} .1 \mathrm{~N} \\
14^{\circ} 03^{\prime} .8 \mathrm{E}\end{array}$ & VI & 4 & 4.6 & $10^{18}$ \\
\hline 85 & 14-IX-" & Amatrice & 一 & IV & 5 & 3.5 & $1.6 \times 10^{16}$ \\
\hline 86 & $\begin{array}{c}7-I I-1909 \\
21^{\mathrm{h}} 15^{\mathrm{m}}\end{array}$ & Montereale & - & V & 5 & 4.1 & $2 \times 10^{17}$ \\
\hline 87 & $16-111-" n$ & L'Aquila & - & IV & 6 & 4.8 & $5 \times 10^{18}$ \\
\hline 88 & $\begin{array}{l}24 \text {-VI- " } \\
23^{\mathrm{h}} 55^{\mathrm{m}}\end{array}$ & $\begin{array}{l}\text { Magliano dei } \\
\text { Marsi }\end{array}$ & - & V & 5 & 4.1 & $2 \times 10^{17}$ \\
\hline 89 & $\begin{array}{c}17-\mathrm{X}-" \prime \\
06^{\mathrm{h}} 16^{\mathrm{m}}\end{array}$ & & - & V & 5 & 4.1 & $2 \times 10^{17}$ \\
\hline 90 & $\begin{array}{c}\text { 4-XI- } \\
06^{\mathrm{h}} \pm ”\end{array}$ & $\begin{array}{l}\text { Montereale } \\
\text { (Gran Sasso }\end{array}$ & - & V & 5 & 4.1 & $2 \times 10^{17}$ \\
\hline 91 & $\begin{array}{c}22.1 I-1910 \\
16^{11} 35^{\mathrm{m}}\end{array}$ & $\begin{array}{l}\text { Magliano de: } \\
\text { Marsi }\end{array}$ & - & VI & 5 & 4.7 & $2.5 \times 10^{18}$ \\
\hline 92 & $\begin{array}{c}\text { 6-III- } \\
19^{\mathrm{h}} \pm\end{array}$ & Accumoli & - & IV & 5 & 3.5 & $1.6 \times 10^{16}$ \\
\hline
\end{tabular}




\begin{tabular}{|c|c|c|c|c|c|c|c|}
\hline $\mathrm{No}^{\circ}$ & Data & Località & $\begin{array}{c}\text { Coord. Ep. } \\
\qquad \quad \lambda\end{array}$ & $I_{o}$ & $\begin{array}{c}h \\
\mathrm{~km}\end{array}$ & $M$ & $\begin{array}{c}E \\
\text { ergs }\end{array}$ \\
\hline 93 & 14-VIII- 1910 & Caramanico & - & $\mathrm{V}$ & 5 & 4.1 & $2 \times 10^{17}$ \\
\hline 94 & $\begin{array}{c}2-\mathrm{XI}_{-} \\
08^{\mathrm{h}} 07^{\mathrm{m}}\end{array}$ & Sulmona & - & IV $-\mathrm{V}$ & 5 & 4.1 & $2 \times 10^{17}$ \\
\hline 95 & $\begin{array}{c}22-X I I-8^{\mathrm{h}} 18^{\mathrm{m}}\end{array}$ & Accumoli & $\begin{array}{ll}42^{\circ} 41^{\prime} & N \\
13^{\circ} 15^{\prime} & \mathrm{E}\end{array}$ & VI & 10 & 5.0 & $2 \times 10^{19}$ \\
\hline 96 & 26-XII-" & $n$ & $" 1$ & VII & 10 & 5.6 & $3.2 \times 10^{20}$ \\
\hline 97 & $\begin{array}{c}21-I V-1911 \\
16^{\mathrm{h}} 32^{\mathrm{m}}\end{array}$ & Caramanico & 一 & IV & 5 & 3.5 & $1.6 \times 10^{16}$ \\
\hline 98 & $\begin{array}{c}28-V . " \\
14^{\mathrm{h}} 52^{\mathrm{m}}\end{array}$ & L'Aquila & - & VI & 6 & 4.2 & $5 \times 10^{18}$ \\
\hline 99 & $\begin{array}{c}9-X I-" ~ \\
12^{\mathrm{h}} 32^{\mathrm{m}}\end{array}$ & $"$ & - & VI & 6 & 4.2 & $5 \times 10^{18}$ \\
\hline 100 & $\begin{array}{c}8-I I I-1912 \\
05^{\mathrm{n}} 04^{\mathrm{m}}\end{array}$ & Paganica & - & IV & 5 & 3.5 & $1.6 \times 10^{18}$ \\
\hline 101 & $\begin{array}{c}3-I-1913 \\
14^{\mathrm{h}} 39^{\mathrm{m}}\end{array}$ & $\begin{array}{l}\text { Gioia dei } \\
\text { Marsi }\end{array}$ & $\begin{array}{l}41^{\circ} 56^{\prime} .6 \mathrm{~N} \\
13^{\circ} 42^{\prime}\end{array}$ & VII & 15 & 5.8 & $10^{21}$ \\
\hline 102 & $\begin{array}{c}4-X-{ }^{\prime \prime} \\
19^{12} 26^{m}\end{array}$ & $\begin{array}{l}\text { Vinchiaturo } \\
\text { - Jelsi }\end{array}$ & $\begin{array}{l}41^{\circ} 30^{\prime} r \\
13^{\circ} 41^{\prime} .2 \mathrm{E}\end{array}$ & VIII & 12 & 6.3 & $6.3 \times 10^{21}$ \\
\hline 103 & $\begin{array}{c}25-\mathrm{XI}-" \prime \\
01^{\mathrm{h}} 57^{\mathrm{m}}\end{array}$ & $\begin{array}{l}\text { Cercemag- } \\
\text { giore }\end{array}$ & $\begin{array}{l}42^{\circ} 27^{\prime}, 4 \mathrm{~N} \\
14^{\circ} 43^{\prime}\end{array}$ & VI & 16 & 5.2 & $10^{20}$ \\
\hline 104 & $\begin{array}{c}14-I V-1914 \\
03^{\mathrm{h}} 49^{\mathrm{m}}\end{array}$ & $\begin{array}{l}\text { Gioia dei M. - } \\
\text { Pescina }\end{array}$ & $\begin{array}{l}42^{\circ} 01^{\prime} .5 \mathrm{~N} \\
13^{\circ} 39^{\prime} .9 \mathrm{E}\end{array}$ & VI & 13 & 5.1 & $5 \times 10^{19}$ \\
\hline 105 & $\begin{array}{l}21-I V . " \\
12^{\mathrm{n}} 46^{\mathrm{m}}\end{array}$ & Avezzano & 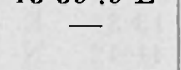 & IV & 5 & 3.5 & $1.6 \times 10^{16}$ \\
\hline 106 & $\begin{array}{c}17-\text { XII- } \\
03^{\mathrm{n}} 16^{\mathrm{m}}\end{array}$ & Isernia & $\begin{array}{l}41^{\circ} 35^{\prime} .3 \mathrm{~N} \\
14^{\circ} 13^{\prime} .2 \mathrm{E}\end{array}$ & VI & 15 & 5.2 & $10^{20}$ \\
\hline 107 & $\begin{array}{c}\text { 19-XII- " } \\
04^{\mathrm{h}} 50^{\mathrm{m}}\end{array}$ & ” & $" 1$ & VII & 8 & 5.5 & $10^{20}$ \\
\hline 108 & $\begin{array}{l}13-I-1915 \\
07^{\mathrm{h}} 52^{\mathrm{m}} 43^{\mathrm{s}}\end{array}$ & Avezzano & $\begin{array}{ll}41^{\circ} 59^{\prime} & \mathrm{N} \\
13^{\circ} 36^{\prime} & \mathrm{E}\end{array}$ & $\mathrm{X}$ & 15 & 7.5 & $10^{23}$ \\
\hline 109 & $\begin{array}{c}13-I I-1916 \\
03^{\mathrm{h}} 20^{\mathrm{m}}\end{array}$ & $\begin{array}{c}\text { Tornim - } \\
\text { parte }\end{array}$ & $\begin{array}{l}42^{\circ} 17^{\prime}, \quad \bar{N} \\
13^{\circ} 17^{\prime} .7 \mathrm{E}\end{array}$ & $\mathrm{V}$ & 10 & 4.4 & $2 \times 10^{18}$ \\
\hline 110 & $\begin{array}{c}20-11-" \\
02^{\mathrm{h}} \pm\end{array}$ & " & " & IV & 10 & 3.8 & $1.6 \times 10^{17}$ \\
\hline 111 & $\begin{array}{c}14-111 . \\
01^{\mathrm{h}} 39^{\mathrm{m}}\end{array}$ & 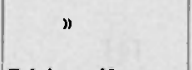 & $" 1$ & VI & 12 & 5.1 & $5 \times 10^{19}$ \\
\hline 112 & $\begin{array}{l}21-\text { IV }-" ~ \\
13^{\mathrm{h}} 46^{\mathrm{m}}\end{array}$ & $\begin{array}{l}\text { L'Aquila - } \\
\text { Tornim- } \\
\text { parte }\end{array}$ & $\begin{array}{ll}42^{\circ} 19^{\prime} & \mathrm{N} \\
13^{\circ} 20^{\prime} & \mathrm{E}\end{array}$ & VI & 8 & 4.9 & $1.3 \times 10^{19}$ \\
\hline 113 & $\begin{array}{c}22.1 \mathrm{~V} . " ” \\
05^{n} 33^{\mathrm{m}}\end{array}$ & L'Aquila - & & V11 & 13 & 5.7 & $8 \times 10^{20}$ \\
\hline 114 & $\begin{array}{l}\text { 4-VII. " } \\
06^{\mathrm{h}} 06^{\mathrm{m}}\end{array}$ & $\begin{array}{l}\text { parte } \\
\text { Trquata del } \\
\text { Tronto }\end{array}$ & $\begin{array}{l}42^{\circ} 46^{\prime} .2 \mathrm{~N} \\
13^{\circ} 17^{\prime} .6 \mathrm{E}\end{array}$ & VIII & 10 & 6.2 & $4.0 \times 10^{21}$ \\
\hline 115 & $\begin{array}{c}28-1 X-" \\
14^{\mathrm{h}} \pm\end{array}$ & L'Áquila & & IV & 5 & 3.5 & $1.6 \times 10^{16}$ \\
\hline 116 & $\begin{array}{c}16 \cdot \mathrm{XI}-" \\
07^{\mathrm{h}} 35^{\mathrm{m}}\end{array}$ & Cittareale & $\begin{array}{l}42^{\circ} 37^{\prime} \quad \mathrm{N} \\
13^{\circ} 09^{\prime} .5 \mathrm{E}\end{array}$ & VIII & 13 & 6.3 & $10^{22}$ \\
\hline 117 & $\begin{array}{r}22 \cdot \mathrm{XI} \cdot \mathrm{\prime} \\
11^{\mathrm{h}} 20^{\mathrm{m}}\end{array}$ & $n$ & $" n$ & $r$ & 20 & 4.7 & $1.6 \times 10^{19}$ \\
\hline
\end{tabular}




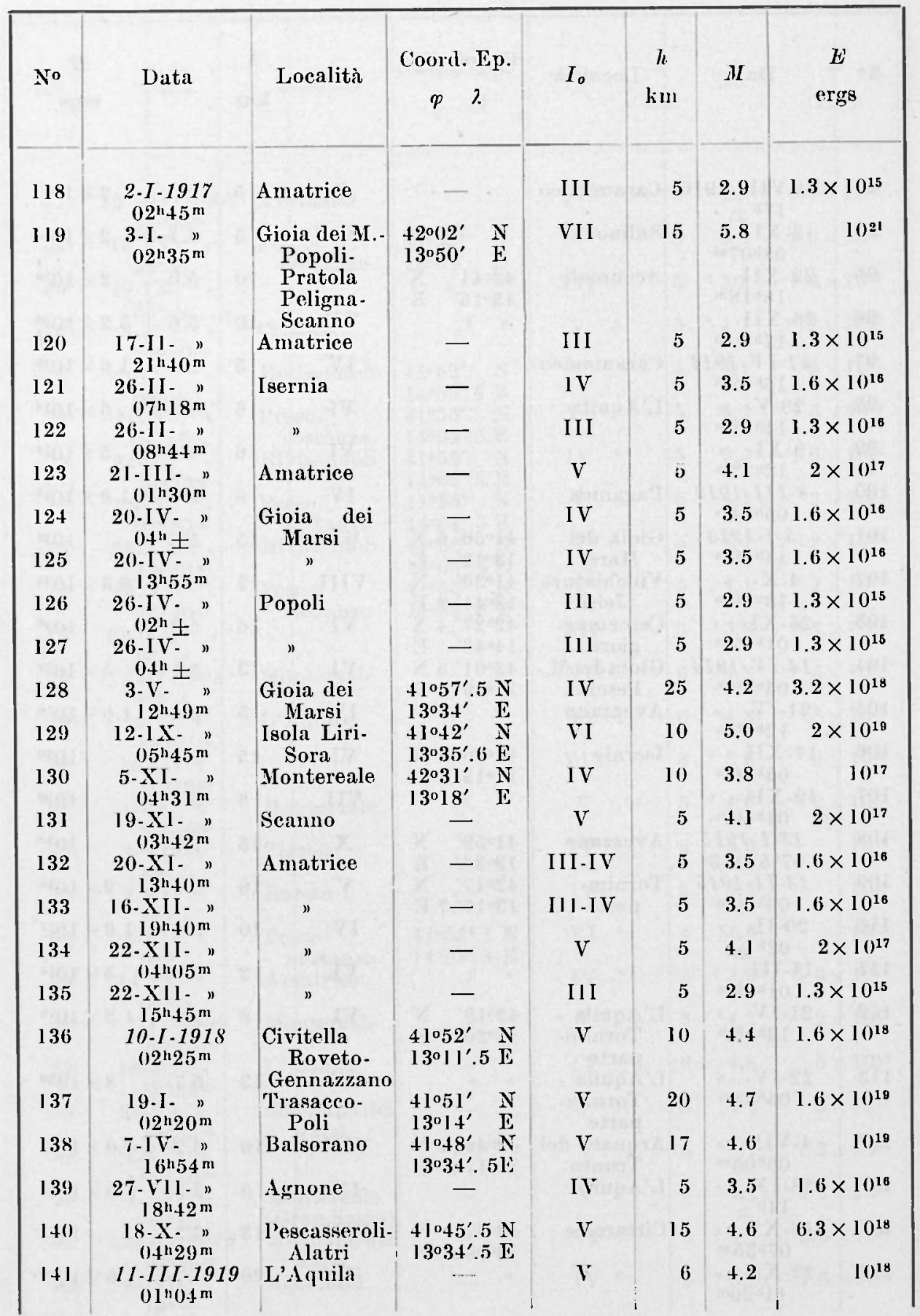




\begin{tabular}{|c|c|c|c|c|c|c|c|}
\hline No & Data & Località & $\begin{array}{c}\text { Coord. Ep. } \\
\varphi \quad \lambda\end{array}$ & $I_{0}$ & $\begin{array}{c}l \\
k \mathrm{~km}\end{array}$ & $M$ & $\begin{array}{c}E \\
\text { ergs }\end{array}$ \\
\hline 142 & $\begin{array}{c}11-111.1919 \\
21 \mathrm{n} 30 \mathrm{~m}\end{array}$ & Borgocolle- & - & IV & 5 & 3.5 & $1.6 \times 10^{16}$ \\
\hline 143 & $\begin{array}{c}20-I V- \\
07^{\mathrm{h}} 32^{\mathrm{m}}\end{array}$ & Avezzano- & $\begin{array}{l}41^{\circ} 39^{\prime}, 6 \mathrm{~N} \\
13^{\circ} 29^{\prime}\end{array}$ & V & 15 & 4.6 & I $0^{19}$ \\
\hline 144 & $\begin{array}{l}\text { 2-VII- " } \\
16^{\mathrm{h}} 30^{\mathrm{m}}\end{array}$ & Venafro & 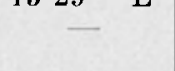 & III & 5 & 2.9 & $1.3 \times 10^{15}$ \\
\hline 145 & $\begin{array}{l}\text { 16-VIII- - } \\
23^{\mathrm{h}} 42^{\mathrm{m}}\end{array}$ & Posta & $\begin{array}{l}42^{\circ} 33^{\prime} .5 \mathrm{~N} \\
13^{\circ} 06^{\prime}\end{array}$ & IV & 10 & 3.8 & $10^{17}$ \\
\hline 146 & $\begin{array}{l}20 \cdot \text { VIII - " } \\
04^{\mathrm{h}} 58^{\mathrm{m}}\end{array}$ & $\begin{array}{c}\text { Castellone al } \\
\text { Volturno }\end{array}$ & $\begin{array}{l}41^{\circ} 39^{\prime} \quad \vec{N} \\
14^{\circ} 03^{\prime} .6 \mathrm{E}\end{array}$ & IV & 10 & 3.8 & $10^{17}$ \\
\hline 147 & $\begin{array}{c}15-\mathrm{IX}-" 口 \\
00^{\mathrm{h}} 08^{\mathrm{m}}\end{array}$ & Amatrice & $\begin{array}{l}42^{\circ} 37^{\prime}, 6 \mathrm{~N} \\
13^{\circ} 17^{\prime} .5 \mathrm{E}\end{array}$ & $\mathrm{V}$ & 9 & 4.4 & $10^{18}$ \\
\hline 148 & $\begin{array}{c}\text { 7-X- " } \\
11^{\mathrm{n}} 28^{\prime \prime \prime}\end{array}$ & Isernia & $\begin{array}{ll}41^{\circ} 35^{\prime} & \mathrm{N} \\
14^{\circ} 14^{\prime} & \mathrm{F}\end{array}$ & IV & 5 & 3.5 & $1.6 \times 10^{16}$ \\
\hline 149 & $\begin{array}{c}15 \cdot \mathrm{X} \cdot " \prime \\
21^{\mathrm{h}} 05^{\mathrm{m}}\end{array}$ & $"$ & $" 1$ & IV & 5 & 3.5 & $1.6 \times 10^{18}$ \\
\hline 150 & $\begin{array}{c}16-\mathrm{X}-" \mathrm{\prime} \\
02^{\mathrm{h}} 10^{\mathrm{m}}\end{array}$ & $"$ & $" n$ & III & 5 & 2.9 & $1.3 \times 10^{15}$ \\
\hline 151 & $\begin{array}{c}16 \cdot \mathrm{X}-" 1 \\
06^{\mathrm{h}} 15^{\mathrm{m}}\end{array}$ & $"$ & $" \#$ & III & 5 & 2.9 & $1.3 \times 10^{15}$ \\
\hline 152 & $\begin{array}{r}24-X-" ~ \\
06^{\mathrm{h}} 08^{\mathrm{m}}\end{array}$ & $”$ & $"$ & IV & 5 & 3.5 & $1.6 \times 10^{18}$ \\
\hline 153 & $\begin{array}{l}2-\mathrm{XI}-" \prime \prime \\
05^{\mathrm{h}} 54^{\mathrm{m}}\end{array}$ & $”$ & $n$ & IV & 5 & 3.5 & $1.6 \times 10^{16}$ \\
\hline 154 & $\begin{array}{c}2-\mathrm{XI}-" \prime \\
16^{\mathrm{h}}+6^{\mathrm{m}}\end{array}$ & $”$ & $n$ & III-I V & 5 & 3.2 & $5 \times \mathrm{IO}^{15}$ \\
\hline 155 & $\begin{array}{c}4 \cdot X I-" ~ \\
12^{n} 11^{m}\end{array}$ & $”$ & $"$ & III-IV & 5 & 3.2 & $5 \times 10^{15}$ \\
\hline 156 & $\begin{array}{c}6-X I- \\
11^{\mathrm{n}} 45^{\mathrm{m}}\end{array}$ & $"$ & $"$ & IV & 5 & 3.5 & $1.6 \times 10^{16}$ \\
\hline 157 & $\begin{array}{c}8 \cdot X I-" 1 \\
14^{n} 26^{\mathrm{m}}\end{array}$ & " & $" 》$ & III-IV & 5 & 3.2 & $5 \times 10^{15}$ \\
\hline 158 & $\begin{array}{c}9-X \mathrm{I}-" ~ \\
05^{\mathrm{h}} 29^{\mathrm{m}}\end{array}$ & ” & $"$ & IV & 5 & 3.5 & $1.6 \times 10^{16}$ \\
\hline 159 & $\begin{array}{c}28-X I . \quad " \\
04^{\mathrm{h}} 25^{\mathrm{m}}\end{array}$ & " & $"$ & IV & 5 & 3.5 & $1.6 \times 10^{16}$ \\
\hline 160 & $\begin{array}{c}29 . \mathrm{Xl} . \mathrm{I}^{\mathrm{h}} 33^{\mathrm{m}}\end{array}$ & ” & $”$ & 11 & 5 & 2.3 & $10^{14}$ \\
\hline 161 & $\begin{array}{c}30-\mathrm{XI} \cdot{ }^{\prime \prime} \\
21^{\mathrm{n}} 10^{\mathrm{m}}\end{array}$ & " & $"$ & 111 & 5 & 2.9 & $1.3 \times 10^{15}$ \\
\hline 162 & $\begin{array}{l}\text { 2-XII- } \\
14^{\mathrm{n}} 05^{\mathrm{m}}\end{array}$ & $"$ & $"$ & IV & 5 & 3.5 & $1.6 \times 10^{16}$ \\
\hline 163 & $\begin{array}{c}\text { 4-XII- } \\
14^{\mathrm{n}} 52^{\mathrm{m}}\end{array}$ & $"$ & $"$ & III & 5 & 2.9 & $1.3 \times 10^{15}$ \\
\hline 164 & $\begin{array}{l}\text { 4-XII- " } \\
15^{\mathrm{h}} 07^{\mathrm{m}}\end{array}$ & $"$ & ” & III & 5 & 2.9 & $1.3 \times 10^{16}$ \\
\hline 165 & $\begin{array}{c}5 \cdot X I I-\quad " \\
01^{\mathrm{h}} 13^{\mathrm{m}}\end{array}$ & $"$ & $n$ & III & 5 & 2.9 & $1.3 \times 10^{15}$ \\
\hline 166 & 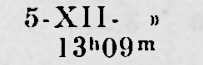 & " & " " & IV & 5 & 3.5 & $1.6 \times 10^{16}$ \\
\hline 167 & $\begin{array}{l}\text { 5.XII- " } \\
15^{\mathrm{h}} 0 \mathrm{I}^{\mathrm{m}}\end{array}$ & $n$ & $n \quad "$ & 111 & 5 & 2.9 & $1.3 \times 10^{15}$ \\
\hline
\end{tabular}




\begin{tabular}{|c|c|c|c|c|c|c|c|}
\hline No & Data & Località & $\begin{array}{c}\text { Coord. Ep. } \\
\varphi \quad \lambda\end{array}$ & $I_{o}$ & $\begin{array}{c}h \\
\mathrm{~km}\end{array}$ & $M$ & $\begin{array}{c}E \\
\operatorname{ergs}\end{array}$ \\
\hline 168 & $\underset{15^{\mathrm{h}} 56^{\mathrm{m}}}{5 \cdot \mathrm{XII} \cdot 1919}$ & Isernia & & IV & 5 & 3.5 & $1.6 \times 10^{16}$ \\
\hline 169 & $\begin{array}{c}\text { 5-XII- " } \\
20^{\mathrm{h}} 26^{\mathrm{m}}\end{array}$ & " & & III & 5 & 2.9 & $1.3 \times 10^{15}$ \\
\hline 170 & $\begin{array}{c}12-\text { XII- } \\
13^{\mathrm{h}} 20^{\mathrm{m}}\end{array}$ & $"$ & $" ”$ & IV.I & 5 & 3.8 & $6.3 \times 10^{16}$ \\
\hline 171 & $\begin{array}{c}13-I-1920 \\
11^{\mathrm{h}} 35^{\mathrm{m}}\end{array}$ & $\begin{array}{c}\text { Tornim- } \\
\text { parte }\end{array}$ & $\begin{array}{l}42^{\circ} 17^{\prime} \quad \mathrm{N} \\
13^{\circ} 17^{\prime} .7 \mathrm{E}\end{array}$ & VI & 10 & 5.0 & $2.5 \times 10^{18}$ \\
\hline 172 & $\begin{array}{c}15-\mathrm{I}-" \\
01^{\mathrm{h}}{ }^{\prime}\end{array}$ & p & $"$ " & VI & 10 & 5.0 & $2.5 \times 10^{19}$ \\
\hline 173 & $\begin{array}{c}11-\mathrm{II}=" \prime \\
01^{\mathrm{b}} 05^{\mathrm{m}}\end{array}$ & Venarotta & $\begin{array}{ll}42^{\circ} 54^{\prime} & \mathrm{N} \\
13^{\circ} 31^{\prime} & \mathrm{E}\end{array}$ & VI & 10 & 5.0 & $2.5 \times 10^{18}$ \\
\hline 174 & $\begin{array}{c}14-\text { XII- } \\
03^{\text {h }} \pm\end{array}$ & $\begin{array}{c}\text { Tornim- } \\
\text { parte }\end{array}$ & $\begin{array}{l}42^{\circ} 17^{\prime} \\
13^{\circ} 17^{\prime} .7 \mathrm{E}\end{array}$ & $\mathrm{V}$ & 10 & 4.4 & $2 \times 10^{18}$ \\
\hline 175 & $\begin{array}{c}28-X I-1922 \\
06^{\mathrm{n}} 56^{\mathrm{m}}\end{array}$ & $\begin{array}{l}\text { L'Aquila - } \\
\text { Sassa - } \\
\text { Borgocol- } \\
\text { lefegato- } \\
\text { Tornim- } \\
\text { parte }\end{array}$ & $\begin{array}{l}42^{\circ} 36^{\prime} .8 \mathrm{~N} \\
13^{\circ} 16^{\prime} .5 \mathrm{E}\end{array}$ & IV & 24 & 4.2 & $2.5 \times 10^{18}$ \\
\hline 176 & $\begin{array}{l}29-X I I-" ~ \\
13^{\mathrm{h}} 22^{\mathrm{m}} 06^{\mathrm{s}}\end{array}$ & $\begin{array}{l}\text { Pescina-- } \\
\text { Gioia dei } \\
\text { Marsi- } \\
\text { Villavalle- } \\
\text { longa- } \\
\text { Balsorano }\end{array}$ & $\begin{array}{ll}41^{\circ} 48^{\prime} & \mathrm{N} \\
13^{\circ} 38^{\prime} & \mathrm{E}\end{array}$ & VII & 26 & 6.0 & $5 \times 10^{21}$ \\
\hline 177 & $\begin{array}{c}13-7-1923 \\
15^{\mathrm{h}} 31^{\mathrm{m}}\end{array}$ & $\begin{array}{c}\text { Borgocolle- } \\
\text { fegato }\end{array}$ & $\begin{array}{l}42^{\circ} 11^{\prime} .5 \mathrm{~N} \\
13^{\circ} 14^{\prime}\end{array}$ & VI & 18 & 5.3 & $10^{20}$ \\
\hline 178 & $\begin{array}{c}\text { 16-VI. " } \\
10^{\mathrm{h}} 59^{\mathrm{m}}\end{array}$ & Balsorano & -- & IV & 5 & 3.5 & $1.6 \times 10^{16}$ \\
\hline 179 & $\begin{array}{c}\text { 29.VI- " } \\
08^{\mathrm{n}} \pm\end{array}$ & $"$ & 一 & Ill-IV & 5 & 3.2 & $5 \times 10^{15}$ \\
\hline 180 & $\begin{array}{c}8-X^{\perp} \\
01^{\mathrm{h}} 43^{\mathrm{m}}\end{array}$ & $\begin{array}{c}\text { Tornim- } \\
\text { parte }\end{array}$ & - & IV & 5 & 3.5 & $1.6 \times 10^{16}$ \\
\hline 181 & $\begin{array}{c}\text { 9-VIII-1924 } \\
23^{\mathrm{h}} 10^{\mathrm{m}}\end{array}$ & Sora & - & $1 \mathrm{~V}-\mathrm{V}$ & 5 & 3.8 & $6 \times 10^{16}$ \\
\hline 182 & $\begin{array}{l}28-I X-" ~ \\
10^{\mathrm{h}} 11^{\mathrm{m}}\end{array}$ & Balsorano & 一 & V-VI & 5 & 4.4 & $8 \times 10^{17}$ \\
\hline 183 & $\begin{array}{c}28-\mathrm{IX}- \\
20^{\mathrm{h}} 58^{\mathrm{m}}\end{array}$ & " & 一 & IV & 5 & 3.5 & $1.6 \times 10^{16}$ \\
\hline 184 & $\begin{array}{c}14-I-1925 \\
20^{\mathrm{h}} 27^{\mathrm{m}}\end{array}$ & Popoli & - & I V $-V$ & 5 & 3.8 & $6 \times 10^{16}$ \\
\hline 185 & $\begin{array}{l}17-\text { II- " } \\
22^{\mathrm{h}} 30^{\mathrm{m}}\end{array}$ & $\begin{array}{l}\text { Lecce dei } \\
\text { Marsi - }\end{array}$ & 一 & IV & 5 & 3.5 & $1.6 \times 10^{16}$ \\
\hline 186 & $\begin{array}{c}9 \cdot \mathrm{VI}-{ }^{\prime \prime} \\
04^{\mathrm{n}} 37^{\mathrm{m}}\end{array}$ & Popoli & - & $\mathrm{V}$ & 5 & 4.1 & $2 \times 10^{17}$ \\
\hline 187 & $\begin{array}{c}24-I X-" ~ \\
14^{\mathrm{h}} 33^{\mathrm{m}}\end{array}$ & $\begin{array}{l}\text { Acquaviva } \\
\text { l'Isernia }\end{array}$ & $\begin{array}{ll}41^{\circ} 40^{\prime} & \mathrm{N} \\
14^{\circ} 09^{\prime} & \mathrm{E}\end{array}$ & VIII & 11 & 6.2 & $4 \times 10^{21}$ \\
\hline 188 & $\begin{array}{c}12-I-1926 \\
07^{\mathrm{h}} 45^{\mathrm{m}}\end{array}$ & $\begin{array}{l}\text { Pizzoli- } \\
\text { Bareto }\end{array}$ & $\begin{array}{l}42^{\circ} 26^{\prime} .5 \mathrm{~N} \\
13^{\circ} 17^{\prime} .7 \mathrm{E}\end{array}$ & IV & 5 & 3.5 & $10^{1 \mathrm{e}}$ \\
\hline 189 & $\begin{array}{l}18-\mathrm{I}- \\
19^{\mathrm{h}} \pm\end{array}$ & Sulmona & - & IV & 5 & 3.5 & $10^{16}$ \\
\hline
\end{tabular}


SISMICITA IN RELAZIONE ALIA TETTONICA E ACCUMUIO DELLE TENSION 333

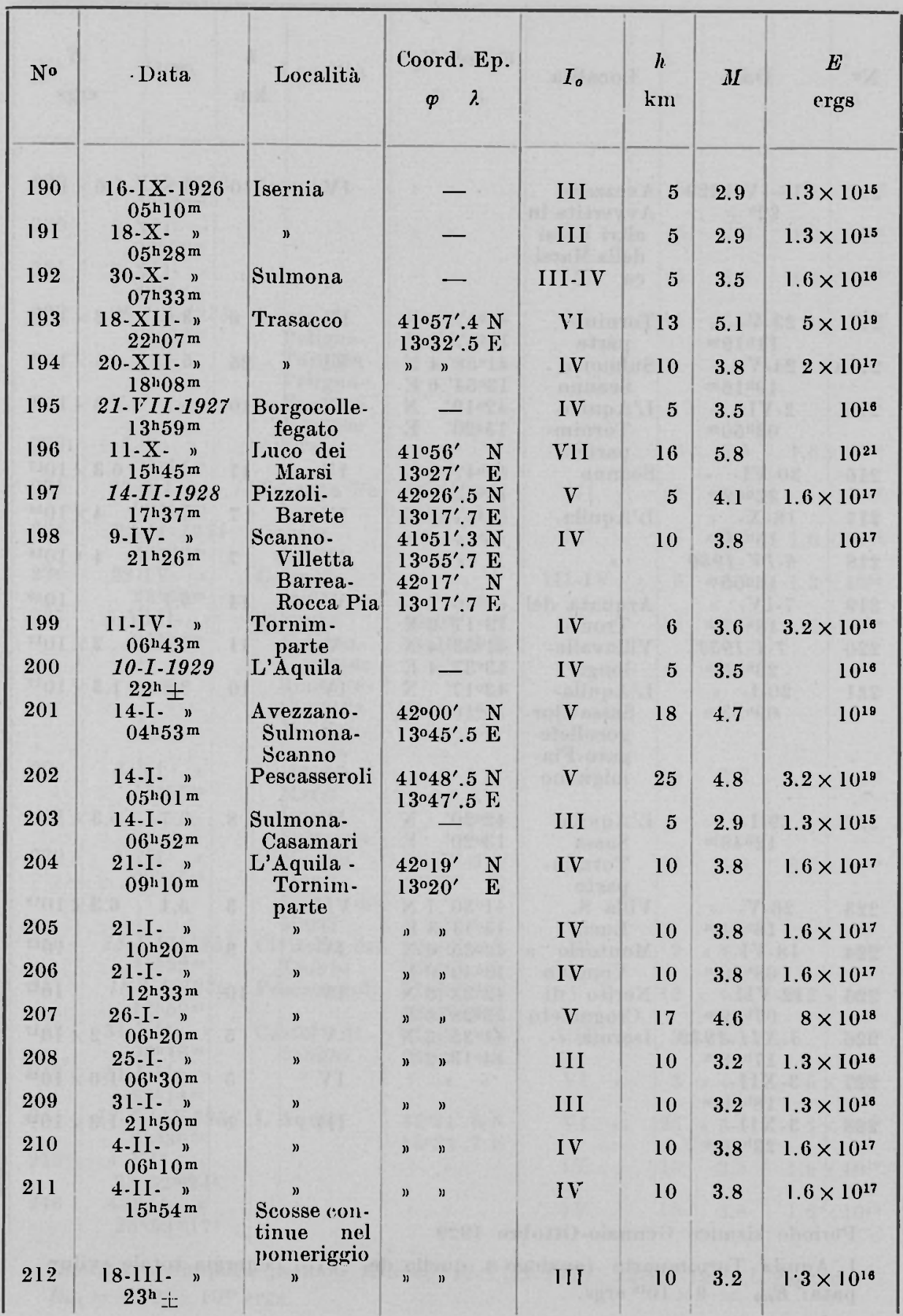




\begin{tabular}{|c|c|c|c|c|c|c|c|}
\hline No & Data & Localitì & $\begin{array}{c}\text { Coord. Ep. } \\
p \quad \lambda .\end{array}$ & $I_{0}$ & $\begin{array}{c}h \\
\mathrm{~km}\end{array}$ & $M$ & $\begin{array}{c}E \\
\text { ergs }\end{array}$ \\
\hline 213 & $\begin{array}{c}\text { 18-IV-1929 } \\
22^{\mathrm{h}} \pm\end{array}$ & $\begin{array}{l}\text { Avezzano } \\
\text { Avvertita in } \\
\text { altri paesi } \\
\text { della Marsi- } \\
\text { ca }\end{array}$ & - & I V & 10 & 3.8 & $1.6 \times 10^{17}$ \\
\hline 214 & $\begin{array}{l}23-V \cdot{ }^{\prime \prime} \\
11^{\mathrm{h}} 19^{\mathrm{m}}\end{array}$ & Tornim- & $\begin{array}{l}42^{\circ} 17^{\prime} \quad \mathrm{N} \\
13^{\circ} 17^{\prime} .7 \mathrm{E}\end{array}$ & IV & 6 & 3.6 & $3.2 \times 10^{16}$ \\
\hline $214 a$ & $\begin{array}{l}24-V . " 1 \\
19^{\mathrm{n}} 16^{\mathrm{m}}\end{array}$ & $\begin{array}{l}\text { Sulmona } \\
\text { Scanno }\end{array}$ & $\begin{array}{l}41^{\circ} 58^{\prime} .4 \mathrm{~N} \\
13^{\circ} 54^{\prime} .6 \mathrm{E}\end{array}$ & VI & 25 & 5.4 & $4 \times 10^{20}$ \\
\hline 215 & $\begin{array}{c}2 \text {-VI- } \\
03^{\mathrm{n}} 50^{\mathrm{m}}\end{array}$ & $\begin{array}{l}\text { L'Aquila- } \\
\text { Tornim- }\end{array}$ & $\begin{array}{ll}42^{\circ} 19^{\prime} & \mathrm{N} \\
13^{\circ} 20^{\prime} & \mathrm{E}\end{array}$ & IV & 10 & 3.2 & $1.3 \times 10^{16}$ \\
\hline 216 & $\begin{array}{l}30-V I-" ~ \\
22^{\mathrm{h}} 42^{\mathrm{m}}\end{array}$ & $\begin{array}{l}\text { parte } \\
\text { Scanno }\end{array}$ & $\begin{array}{l}41^{\circ} 47^{\prime} .5 \mathrm{~N} \\
13^{\circ} 52^{\prime} .3 \mathrm{E}\end{array}$ & IV & 17 & 4.0 & $6.3 \times 10^{17}$ \\
\hline 217 & $\begin{array}{c}18-X_{-} " \\
15^{\mathrm{h}} 09^{\mathrm{m}}\end{array}$ & L'Aquila & $\begin{array}{l}42021^{\prime} .6 \mathrm{~N} \\
13^{\circ} 24^{\prime} .7 \mathrm{E}\end{array}$ & IV & 7 & 3.6 & $4 \times 10^{16}$ \\
\hline 218 & $\begin{array}{c}6-I V-1930 \\
16^{\mathrm{h}} 05^{\mathrm{m}}\end{array}$ & $"$ & $" n$ & IV & 7 & 3.6 & $4 \times 10^{16}$ \\
\hline 219 & $\begin{array}{c}\text { 7-IV- } " ~ \\
18^{\mathrm{h}} 17^{\mathrm{m}}\end{array}$ & $\begin{array}{c}\text { Arquata del } \\
\text { Tronto }\end{array}$ & $\begin{array}{l}42^{\circ} 46^{\prime} .2 \mathrm{~N} \\
13^{\circ} 17^{\prime} .6 \mathrm{E}\end{array}$ & Vl & 14 & 5.1 & $10^{20}$ \\
\hline 220 & $\begin{array}{c}\tau-I-1931 \\
23^{\mathrm{h}} 45^{\mathrm{m}}\end{array}$ & $\begin{array}{l}\text { Villavalle- } \\
\text { longa }\end{array}$ & $\begin{array}{l}41^{\circ} 53^{\prime} .4 \mathrm{~N} \\
13^{\circ} 37^{\prime} .4 \mathrm{E}\end{array}$ & $V$ & 11 & 4.4 & $2 \times 10^{18}$ \\
\hline 221 & $\begin{array}{c}20-1 . \\
09^{\mathrm{h}} 53^{\mathrm{m}}\end{array}$ & $\begin{array}{l}\text { L'A quila- } \\
\text { Sassa-Bor- } \\
\text { gocollefe- } \\
\text { gato-Fia- } \\
\text { mignano }\end{array}$ & $\begin{array}{l}42^{\circ} 17^{\prime} \mathrm{N} \\
13^{\circ} 16^{\prime} .9 \mathrm{E}\end{array}$ & IV & 10 & 3.8 & $1.3 \times 10^{17}$ \\
\hline 222 & $\begin{array}{l}29-1-" ~ \\
12^{\mathrm{h}} 48^{\mathrm{m}}\end{array}$ & $\begin{array}{l}\text { L'Aquila- } \\
\text { Sassa } \\
\text { Tornim- }\end{array}$ & $\begin{array}{ll}42^{\circ} 20^{\prime} & \mathrm{N} \\
13^{\circ} 20^{\prime} & \mathrm{E}\end{array}$ & IV & 8 & 3.7 & $6.3 \times 10^{16}$ \\
\hline 223 & $\begin{array}{l}26 \cdot V \cdot 1 " \\
16^{\mathrm{h}} 24^{\mathrm{m}}\end{array}$ & $\begin{array}{l}\text { Villa S. } \\
\text { Lucia }\end{array}$ & $\begin{array}{l}41^{\circ} 30^{\prime} .7 \mathrm{~N} \\
13^{\circ} 13^{\prime} .3 \mathrm{E}\end{array}$ & VII & 3 & 5.1 & $6.3 \times 10^{18}$ \\
\hline 224 & $\begin{array}{l}\text { 18-VI- " } \\
08^{\prime \prime} 20^{m}\end{array}$ & $\begin{array}{c}\text { Montorio a } \\
\text { Vomano }\end{array}$ & $\begin{array}{l}42^{\circ} 35^{\prime} .0 \mathrm{~N} \\
13^{\circ} 40^{\prime} .0 \mathrm{E}\end{array}$ & IV & 9 & 3.8 & $10^{17}$ \\
\hline 225 & $\begin{array}{c}\text { 12-VII- " } \\
07^{\mathrm{h}} 19^{\mathrm{m}}\end{array}$ & $\begin{array}{l}\text { Nerito di } \\
\text { Crognaleto }\end{array}$ & $\begin{array}{l}42^{\circ} 32^{\prime} .6 \mathrm{~N} \\
13^{\circ} 28^{\prime} .6 \mathrm{E}\end{array}$ & IV & 10 & 3.8 & $10^{17}$ \\
\hline 226 & $\begin{array}{c}3-X I I-1932 \\
17^{\mathrm{h}} 58^{\mathrm{m}}\end{array}$ & Isernia & $\begin{array}{l}41^{\circ} 35^{\prime} .3 \mathrm{~N} \\
14^{\circ} 13^{\prime} .2 \mathrm{E}\end{array}$ & V & 5 & 4.1 & $2 \times 10^{17}$ \\
\hline 227 & $\begin{array}{c}\text { 3-XII- " } \\
18^{\mathrm{h}} 50 \mathrm{~m}\end{array}$ & $n$ & " & I V & 5 & 3.5 & $1.6 \times 10^{16}$ \\
\hline 228 & $\begin{array}{l}\text { 3-XII- " } \\
23^{\mathrm{h}} 50^{\mathrm{m}}\end{array}$ & $"$ & $" 1$ & I I I & 5 & 2.9 & $1.3 \times 10^{15}$ \\
\hline
\end{tabular}

Periodo sismico Gennaio-()ttobre 1929

L'A Aquila - Tornimparte (analogo a quello del 1916) l'energia totale sviluppata: $E_{\text {tot }}=9 \times 10^{18} \mathrm{ergs}$. 
SISMICIT i IN RELAzIONe ALLA TETTONICA E ACCUMUlo DELLE TExSION] 335

\begin{tabular}{|c|c|c|c|c|c|c|c|}
\hline No & Data & Località & $\begin{array}{c}\text { Coord. Ep. } \\
\varphi \lambda\end{array}$ & $I_{0}$ & $\begin{array}{c}h \\
\mathrm{~km}\end{array}$ & $M$ & $\begin{array}{c}E \\
\text { ergs }\end{array}$ \\
\hline 229 & $\underset{02^{\mathrm{h}} 59^{\mathrm{m}}}{+ \text { XII- }}$ & Isernia & $" n$ & $\mathrm{~V}$ & 5 & 4.1 & $2 \times 10^{17}$ \\
\hline 230 & $\begin{array}{c}5 \cdot \text { XII- } \\
\left.02^{\mathrm{n}} 50\right)^{\mathrm{m}}\end{array}$ & $"$ & $" 1$ & III & $\bar{\jmath}$ & 2.9 & $1.3 \times 10^{15}$ \\
\hline 231 & $\begin{array}{c}\text { 5-XII- " } \\
19^{\mathrm{h}} 14^{\mathrm{m}}\end{array}$ & $"$ & $" n$ & 111 & 5 & 2.9 & $1.3 \times 10^{15}$ \\
\hline 232 & $\begin{array}{c}26-I X-1933 \\
04^{\mathrm{h}} 33^{\mathrm{m}}\end{array}$ & $\begin{array}{l}\text { Lama di } \\
\text { Peligna- } \\
\text { Taranta } \\
\text { Peligna- } \\
\text { Fara S. } \\
\text { Jartino }\end{array}$ & $\begin{array}{l}42^{\circ} 03^{\prime} .0 \mathrm{~N} \\
14^{\circ} 11^{\prime} .4 \mathrm{E}\end{array}$ & IX & 14 & 6.95 & $10^{23}$ \\
\hline 233 & $\begin{array}{c}7-X-1 " \\
07^{\mathrm{h}} 14^{\mathrm{m}}\end{array}$ & Arsita & - & I V & 5 & 3.5 & $1.6 \times 10^{16}$ \\
\hline 234 & $\begin{array}{c}23-\mathrm{XI}-" ~ \\
02^{\mathrm{h}} 13^{\mathrm{m}}\end{array}$ & $\begin{array}{c}\text { Taranta } \\
\text { ligna }\end{array}$ & $\begin{array}{l}42^{\circ} 01^{\prime} .1 \mathrm{~N} \\
14^{\circ} 10^{\prime} .3 \mathrm{E}\end{array}$ & VII & 17 & 5.8 & $10^{21}$ \\
\hline 235 & $\begin{array}{c}22 \cdot I I-1934 \\
13^{\mathrm{n}} 26^{\mathrm{m}}\end{array}$ & Isernia & & IV & 5 & 3,5 & $1.6 \times 10^{16}$ \\
\hline 236 & $\begin{array}{c}22-1 \mathrm{~V} . \\
23^{\mathrm{h}} 50^{\mathrm{m}}\end{array}$ & $\begin{array}{l}\text { Guardia- } \\
\text { grele }\end{array}$ & 一 & III-IV & 5 & 2.9 & $1.3 \times 10^{15}$ \\
\hline 237 & $\begin{array}{c}\text { 5-V11I- } \\
01^{\mathrm{h}} 50^{\mathrm{m}}\end{array}$ & $\begin{array}{l}\text { Taranta } \\
\text { Peligna- } \\
\text { Pernapie } \\
\text { dimonte- } \\
\text { Civitella } \\
\text { M. Rai- } \\
\text { mondo }\end{array}$ & $\begin{array}{l}42^{\circ} 04^{\prime} \quad \mathrm{N} \\
14^{\circ} 10^{\prime} .2 \mathrm{E}\end{array}$ & V & 14 & 4.5 & $5 \times 10^{18}$ \\
\hline 238 & $\begin{array}{l}3-\mathrm{XI}-{ }^{\prime \prime} \\
11^{\mathrm{h}} 35^{\mathrm{m}}\end{array}$ & $\begin{array}{l}\text { Gioia dei } \\
\text { Marsi- } \\
\text { Luco- } \\
\text { Sulmona }\end{array}$ & - & III & 10 & 3.2 & $10^{16}$ \\
\hline 239 & $\begin{array}{c}4-X 1-" ~ \\
02^{n} 41^{m}\end{array}$ & $\begin{array}{l}\text { Gioia dei } \\
\text { Marsi } \\
\text { Luco dei } \\
\text { Marsi }\end{array}$ & $\begin{array}{ll}41^{\circ} 57^{\prime} .6 \mathrm{~N} \\
13^{\circ} 35^{\prime}\end{array}$ & V & 25 & 4.8 & $3.2 \times 10^{19}$ \\
\hline 240 & $\begin{array}{c}24.111-193 j \\
15^{\mathrm{h}} 32^{\mathrm{m}}\end{array}$ & $\begin{array}{c}\text { Civitella del } \\
\text { Tronto }\end{array}$ & $\begin{array}{l}42^{\circ} 46^{\prime} .6 \mathrm{~N} \\
13^{\circ} 40^{\prime} .4 \mathrm{E}\end{array}$ & V & 9 & 4.4 & $10^{18}$ \\
\hline 241 & $\begin{array}{c}18-\mathrm{V} I-1936 \\
12^{\mathrm{h}} 52^{\mathrm{m}}\end{array}$ & Pescasseroli & $\begin{array}{l}41^{\circ}+8^{\prime} .6 \mathrm{~N} \\
13^{\circ} 47^{\prime} .3 \mathrm{E}\end{array}$ & V & 12 & 4.5 & $3.2 \times 10^{18}$ \\
\hline 242 & $\begin{array}{c}31-V I I- \\
06^{\mathrm{h}}+6^{\mathrm{m}}\end{array}$ & $\begin{array}{l}\text { Castel di } \\
\text { Sangro }\end{array}$ & $\begin{array}{ll}41^{\circ} 47^{\prime} & N \\
14^{\circ} 08^{\prime} & \mathrm{E}\end{array}$ & VII & 3 & 5.1 & $10^{18}$ \\
\hline 243 & $\begin{array}{c}\text { 31-VII- " } \\
08^{\mathrm{h}} 14^{\mathrm{m}}\end{array}$ & 10 & $" n$ & VI & 3 & 4.5 & $5 \times 10^{17}$ \\
\hline 244 & $\begin{array}{c}12-\mathrm{T} I I I-1938 \\
08^{\mathrm{n}} 30^{\mathrm{m}}\end{array}$ & L'Aquila. & $\begin{array}{l}42^{\circ} 21^{\prime} .6 \mathrm{~N} \\
13024^{\prime} .7 \mathrm{~K}\end{array}$ & VI & 25 & 5.4 & $4 \times 10^{20}$ \\
\hline 245 & $\begin{array}{l}\text { 4-XII- } \\
23^{\mathrm{h}} 52^{\mathrm{m}} 24^{\mathrm{s}}\end{array}$ & " & " & IV & 10 & 3.8 & $1.6 \times 10^{17}$ \\
\hline 246 & $\begin{array}{l}4-\text { XII } " \\
23 \mathrm{~h} 59^{\mathrm{m}} 17^{\mathrm{s}}\end{array}$ & " & $"$ & IV & 10 & 3.8 & $1.6 \times 10^{17}$ \\
\hline
\end{tabular}

Energia sviluppata periodo sismico I2-VIII.1938; 12-1I1-I939 L'Aquili.

$E_{\text {tot }}=4.025 \times 10^{20} \mathrm{ergs}$. 


\begin{tabular}{|c|c|c|c|c|c|c|c|}
\hline No & Data & Località & $\begin{array}{c}\text { Coord. Ep. } \\
\qquad \lambda\end{array}$ & $I_{0}$ & $\begin{array}{c}h \\
\mathrm{~km}\end{array}$ & $M I$ & $\begin{array}{c}E \\
\text { ergs }\end{array}$ \\
\hline 247 & $\begin{array}{c}5-\text { XII-1938 } \\
00^{\mathrm{h}} 03^{\mathrm{m}} 45^{\mathrm{s}}\end{array}$ & L'Aquila & $\begin{array}{l}42^{\circ} 21^{\prime} \cdot 0 \mathrm{~N} \\
13^{\circ} 24^{\prime} \cdot 7 \mathrm{E}\end{array}$ & IV & 10 & 3.8 & $1.6 \times 10^{17}$ \\
\hline 248 & $\begin{array}{c}12-I I I-1939 \\
17^{\mathrm{h}} 12^{\mathrm{m}} 55^{\mathrm{s}}\end{array}$ & 》 & $"$ & $\mathrm{~V}$ & 10 & 4.4 & $2 \times 10^{18}$ \\
\hline 249 & $\begin{array}{l}11-\text { VII- " } \\
04^{\mathrm{h}} 47^{\mathrm{m}} 3^{\mathrm{s}}\end{array}$ & Chieti & $\begin{array}{l}42^{\circ} 21^{\prime} .0 \mathrm{~N} \\
14^{\circ} 10^{\prime} .0 \mathrm{E}\end{array}$ & $\mathrm{V}$ & 10 & 4.4 & $2 \times 10^{18}$ \\
\hline 250 & $\begin{array}{c}9-I-1940 \\
07^{\mathrm{h}} 47^{\mathrm{m}}\end{array}$ & $\begin{array}{l}\text { Pettorano } \\
\text { sul Gizio }\end{array}$ & - & IV & 5 & 3.5 & $10^{16}$ \\
\hline 251 & $\begin{array}{c}10-1-" \\
07^{\mathrm{h}} \pm\end{array}$ & $\begin{array}{l}\text { Popoli- } \\
\text { Scanno- } \\
\text { Pesco- }\end{array}$ & $\begin{array}{l}42^{\circ} 01^{\prime}, 2 \mathrm{~N} \\
13^{\circ} 55^{\prime}\end{array}$ & IV & 10 & 3.8 & $1.6 \times 10^{16}$ \\
\hline 252 & $\begin{array}{c}16-11-" ~ \\
03^{\text {h }} 05^{\mathrm{m}}\end{array}$ & $\begin{array}{r}\text { costanzo } \\
\text { Antrodoco }\end{array}$ & 一 & IV - V & 5 & 3.8 & $6.3 \times 10^{16}$ \\
\hline 253 & $\begin{array}{c}9-\text { III- } \\
00^{\mathrm{h}} 33^{\mathrm{m}}\end{array}$ & $\begin{array}{l}\text { Isernia - } \\
\text { Frosolone }\end{array}$ & - & IV & 5 & 3.5 & $1.6 \times 10^{16}$ \\
\hline 254 & $\begin{array}{l}27-111-" \\
00^{\mathrm{h}} 32^{\mathrm{m}}\end{array}$ & Isernia & - & IV & 5 & 3.5 & $1.6 \times 10^{16}$ \\
\hline 255 & $\begin{array}{c}15-X . X " \\
09^{n} 51 \mathrm{~m}\end{array}$ & $\begin{array}{c}\text { Castel di } \\
\text { Sangro }\end{array}$ & 一 & V.VI & 5 & 4.5 & $5 \times 10^{17}$ \\
\hline 256 & $\begin{array}{l}24-X I-" ~ \\
16^{\mathrm{h}} 30,11^{\mathrm{m}}\end{array}$ & $n$ & - & V-VI & 5 & 4.5 & $5 \times 10^{17}$ \\
\hline 257 & $\begin{array}{l}14-I I-1941 \\
12^{\mathrm{h}} 04,03 \mathrm{~m}\end{array}$ & $\begin{array}{l}\text { Monte- } \\
\text { cassino }\end{array}$ & - & IV & 5 & 3.5 & $1.6 \times 10^{16}$ \\
\hline 258 & $\begin{array}{l}14-I V- \\
04^{\mathrm{h}} 22,5^{\mathrm{m}}\end{array}$ & $\begin{array}{l}\text { S. Anatolia } \\
\text { (Velino) }\end{array}$ & $\begin{array}{ll}42^{\circ} 45^{\prime} & \mathrm{N} \\
12^{\circ} 45^{\prime} & \mathrm{E}\end{array}$ & $\mathrm{V}$ & 7 & 4.2 & $6 \times 10^{17}$ \\
\hline 259 & $\begin{array}{c}14-\mathrm{IV}-" 口 \\
12^{\mathrm{n}} 22,5^{\mathrm{m}}\end{array}$ & " & - & $\mathrm{V}$ & 7 & 4.2 & $6 \times 10^{17}$ \\
\hline 260 & $\begin{array}{l}14-\mathrm{IV} \cdot " \\
12^{\mathrm{h}} 23,8^{\mathrm{m}}\end{array}$ & $n$ & $\begin{array}{ll}42^{\circ} 45^{\prime} & \mathrm{N} \\
12^{\circ} 45^{\prime} & \mathrm{E}\end{array}$ & $\mathrm{V}$ & 7 & 4.2 & $6 \times 10^{17}$ \\
\hline 261 & $\begin{array}{c}14-\mathrm{IV}-" \\
16^{\mathrm{h} 01 \mathrm{~m}}\end{array}$ & $"$ & " & V & 7 & 4.2 & $6 \times 10^{17}$ \\
\hline 262 & $14-\mathrm{IV}-{ }_{16^{\mathrm{h}} 16^{\mathrm{m}}}$ & 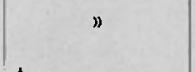 & $" n$ & V & 7 & 4.2 & $6 \times 10^{17}$ \\
\hline 263 & $\begin{array}{l}26 \text {-VII - " } \\
04^{\mathrm{h}} 10^{\mathrm{m}}\end{array}$ & Avezzano & $\begin{array}{l}42^{\circ} 01^{\prime} .8 \mathrm{~N} \\
13^{\circ} 28^{\prime} .5 \mathrm{E}\end{array}$ & I $r$ & 17 & 4.0 & $6.3 \times 10^{17}$ \\
\hline 264 & $\begin{array}{l}\text { 1.XII- } " ~ \\
04^{\mathrm{h}} 10^{\mathrm{m}}\end{array}$ & L'Aquila & $" n$ & IV & 7 & 3.6 & $5 \times 10^{17}$ \\
\hline 265 & $\begin{array}{c}20-V I-1942 \\
07^{\mathrm{h}} 48^{\mathrm{m}}\end{array}$ & $\begin{array}{l}\text { L'Aquila- } \\
\text { Monterea- } \\
\text { le-Sassa }\end{array}$ & $\begin{array}{l}42^{\circ} 26^{\prime} .3 \mathrm{~N} \\
13^{\circ} 18^{\prime} .1 \mathrm{E}\end{array}$ & $1 \mathrm{~V}$ & 10 & 3.8 & $1.6 \times 10^{17}$ \\
\hline 266 & $\begin{array}{c}25 \text {-VII- } \\
20^{\mathrm{h}} 40^{\mathrm{m}}\end{array}$ & Alvito & $\begin{array}{l}41^{\circ} 41^{\prime} .2 \mathrm{~N} \\
13^{\circ} 44^{\prime} .6 \mathrm{E}\end{array}$ & $V$ & 5 & 4.2 & $2 \times 10^{17}$ \\
\hline 267 & $\begin{array}{c}4-X I- \\
03^{\mathrm{h}} 19^{\mathrm{m}}\end{array}$ & $\begin{array}{l}\text { L'Aquila- } \\
\text { Sassa }\end{array}$ & $\begin{array}{ll}42^{\circ} 21^{\prime} & \mathrm{N} \\
13^{\circ} 21^{\prime} & \mathrm{E}\end{array}$ & IV & 10 & 3.8 & $1.6 \times 10^{17}$ \\
\hline 268 & $\begin{array}{c}29-I-1943 \\
04^{\mathrm{h}} 23^{\mathrm{m}}\end{array}$ & $\begin{array}{l}\text { Teramo- } \\
\text { Accumoli- } \\
\text { Cittareale }\end{array}$ & $\begin{array}{l}42^{\circ} 39^{\prime} 21^{\prime \prime} \mathrm{N} \\
13^{\circ} 29^{\prime} 31^{\prime \prime} \mathrm{E}\end{array}$ & Vl & 25 & 5.4 & $4 \times 10^{20}$ \\
\hline 269 & $\begin{array}{c}15-V I I-1948 \\
16^{\mathrm{h}} \pm\end{array}$ & Bolognano & $\begin{array}{l}42^{\circ} 13^{\prime} \quad \mathrm{N} \\
13^{\circ} 57^{\prime} .8 \mathrm{E}\end{array}$ & V & 7 & 4.2 & $5 \times 10^{17}$ \\
\hline 270 & $\begin{array}{c}15 \text {-VII- } \\
23^{\mathrm{h}} 45^{\mathrm{m}}\end{array}$ & Alanno & $\begin{array}{l}\left.42^{\circ}\right] 7^{\prime} .3 \mathrm{~N} \\
13^{\circ} 58^{\prime} .5 \mathrm{E}\end{array}$ & $Y$ & 6 & 4.2 & $4 \times 10^{17}$ \\
\hline
\end{tabular}




\begin{tabular}{|c|c|c|c|c|c|c|c|}
\hline No & Data & Jocalità & $\begin{array}{c}\text { Coord. Ep. } \\
\varphi \quad \lambda\end{array}$ & $I_{o}$ & $\begin{array}{c}h \\
k m\end{array}$ & $M$ & $\begin{array}{c}E^{\prime} \\
\text { ergs }\end{array}$ \\
\hline 271 & $\begin{array}{c}12-I I I-19.50 \\
05^{11} 10^{\mathrm{m}}\end{array}$ & Accumoli & & VII & 10 & 5.6 & $3.2 \times 10^{20}$ \\
\hline 272 & $\begin{array}{l}\text { 5-IX- " } \\
05^{\text {h }} 08^{\mathrm{m}} 57^{\mathrm{s}}\end{array}$ & Gran Sasso & $\begin{array}{l}42^{\circ} 31^{\prime} \quad \mathrm{N} \\
13^{\circ} 19^{\prime} .6 \mathrm{E}\end{array}$ & $1 \mathrm{X}$ & 22 & 7.1 & $5 \times 10^{23}$ \\
\hline 273 & $\begin{array}{l}18-I X-" ~ \\
01^{\mathrm{h}} 40^{\mathrm{m}}\end{array}$ & $\begin{array}{l}\text { Montereale } \\
\text { (Gran Sasso) }\end{array}$ & & VI & 10 & 5.0 & $2.5 \times 10^{19}$ \\
\hline 274 & 2. $P I I I-19.51$ & $\begin{array}{c}\text { Torricella } \\
\text { Sicura }\end{array}$ & - & V & 5 & 3.5 & $1.6 \times 10^{16}$ \\
\hline 275 & $\begin{array}{l}\text { 8-VIII- } \\
21^{\mathrm{h}} 56^{\mathrm{m}}\end{array}$ & Canzano & $\begin{array}{ll}42^{\circ} 36^{\prime} & \mathrm{N} \\
13^{\circ} 30^{\prime} & \mathrm{E}\end{array}$ & VIII & 19 & 6.5 & $2.5 \times 10^{22}$ \\
\hline 276 & $\begin{array}{l}\text { 8-VIII- " } \\
22^{\mathrm{h}} 1 \mathrm{~m}^{\mathrm{m}} \\
23^{\mathrm{h}} 09_{\mathrm{m}}^{\mathrm{m}}\end{array}$ & $\begin{array}{c}\text { Repliche } \\
, \\
\eta\end{array}$ & " " & $\begin{array}{l}\text { VI } \\
n \\
n\end{array}$ & $\begin{array}{l}10 \\
11 \\
11\end{array}$ & $\begin{array}{c}5.0 \\
\prime \prime\end{array}$ & $\begin{array}{c}2.5 \times 10^{18} \\
" \prime \prime\end{array}$ \\
\hline 277 & $\begin{array}{l}23^{\mathrm{h}} 53^{\mathrm{m}} \\
\text { 9.VIII- } \\
\text { OII }\end{array}$ & " & " & $"$ & " & " & " " " \\
\hline & $\begin{array}{l}00^{\mathrm{h}} 59^{\mathrm{m}} \\
05^{\mathrm{h}} 45^{\mathrm{m}}\end{array}$ & " & 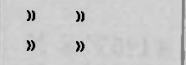 & " & " & ” & $\begin{array}{l}1 " \prime \\
" 1 "\end{array}$ \\
\hline & $\begin{array}{c}06^{n} 45^{m} \\
15^{n} 13^{m}\end{array}$ & " & " & $"$ & $"$ & $"$ & " " " \\
\hline 278 & $\begin{array}{c}24-X I-1953 \\
04^{\mathrm{h}} 13^{\mathrm{m}}\end{array}$ & Avezzano & $\begin{array}{l}42^{\circ} 01^{\prime} .9 \mathrm{~N} \\
13^{\circ} 25^{\prime} .6 \mathrm{E}\end{array}$ & V & 10 & 4.4 & $1.6 \times 10^{18}$ \\
\hline 279 & $\begin{array}{c}3-X I I-" ~ \\
06^{\mathrm{h}} 56^{\mathrm{m}} 08^{\mathrm{s}}\end{array}$ & Colletorto & $\begin{array}{ll}41^{\circ} 42^{\prime} & \mathrm{N} \\
14^{\circ} 48^{\prime} & \mathrm{E}\end{array}$ & V & 10 & 4.4 & $1.6 \times 10^{18}$ \\
\hline 280 & $\begin{array}{c}21-X I I-1954 \\
02^{\mathrm{h}} 30^{\mathrm{m}}\end{array}$ & Campli & -- & V & 18 & 4.7 & $10^{19}$ \\
\hline 281 & $\begin{array}{c}3 \cdot X^{X}-1956 \\
01^{\mathrm{h}} 34^{\mathrm{m}}\end{array}$ & $\begin{array}{c}\text { Caramanico- } \\
\text { Tocco } \\
\text { Casauria }\end{array}$ & $\begin{array}{ll}42^{\circ} 12^{\prime} & \mathrm{N} \\
13^{\circ} 59^{\prime} & \mathrm{E}\end{array}$ & V & 10 & 4.4 & $1.6 \times 10^{18}$ \\
\hline 282 & $\begin{array}{c}25-\mathrm{XI}-" \prime \prime \\
03^{\mathrm{h}} 06^{\mathrm{m}} 40^{\mathrm{s}}\end{array}$ & $\begin{array}{l}\text { Macchiago- } \\
\text { dena }\end{array}$ & $\begin{array}{ll}41^{\circ} 35^{\prime} & \mathrm{N} \\
14^{\circ} 24^{\prime} .4 & \mathrm{E}\end{array}$ & VI & 19 & 5.3 & $10^{20}$ \\
\hline 283 & $\begin{array}{c}29-\mathrm{XI}-{ }^{\prime \prime} \\
03^{\mathrm{h}} 15^{\mathrm{m}}\end{array}$ & $\begin{array}{c}\text { Macchiago- } \\
\text { dena- S. } \\
\text { Angelo in } \\
\text { Grotte }\end{array}$ & $\begin{array}{l}41033^{\prime} .6 \mathrm{~N} \\
14^{\circ} 23^{\prime} .5 \mathrm{~F}\end{array}$ & V & 15 & 4.6 & $6.3 \times 10^{18}$ \\
\hline 284 & $\begin{array}{l}\text { 2-XII- } " ~ \\
09^{\mathrm{n}} 35^{\mathrm{m}}\end{array}$ & S. Angelo in & $\begin{array}{l}41^{\circ} 35^{\prime}, 8 \mathrm{~N} \\
14^{\circ} 21^{\prime} \mathrm{F}\end{array}$ & V & 7 & 4.2 & $5 \times 10^{17}$ \\
\hline 285 & $\begin{array}{r}25 \cdot I-1957 \\
05^{\mathrm{h}} 40^{\mathrm{m}}\end{array}$ & $\begin{array}{l}\text { Pizzoli- } \\
\text { Barete }\end{array}$ & $\begin{array}{l}42^{\circ} 26^{\prime} .5 \mathrm{~N} \\
13^{\circ} 17^{\prime} .5 \mathrm{E}\end{array}$ & VI & 17 & 4.6 & $8 \times 10^{18}$ \\
\hline 286 & $\begin{array}{c}11-\mathrm{IV}-" \prime \\
17^{\mathrm{h}} 19^{\mathrm{m}}\end{array}$ & $\begin{array}{l}\text { Petrella } \\
\text { Salto-Var- } \\
\text { co Sabino }\end{array}$ & $\begin{array}{l}42^{\circ} 15^{\prime} .7 \mathrm{~N} \\
13^{\circ} 02^{\prime} .4 \mathrm{E}\end{array}$ & V1 & 12 & 5.1 & $4 \times 10^{19}$ \\
\hline 287 & $\begin{array}{c}21-I V-1958 \\
03^{\mathrm{h}} 22^{\mathrm{m}} 40^{\mathrm{s}}\end{array}$ & $\begin{array}{l}\text { Montorio } \\
\text { al Voma- } \\
\text { no-Campli }\end{array}$ & $\begin{array}{l}42^{\circ} 39^{\prime}, \mathrm{N} \\
13^{\circ} 39^{\prime} .3 \mathrm{E}\end{array}$ & V & 18 & 4.7 & $10^{18}$ \\
\hline 288 & 26 IV $03^{\mathrm{h}} 20^{\mathrm{m}}$ & $\begin{array}{l}\text { Torricella } \\
\text { Sicura }\end{array}$ & $\begin{array}{l}42^{\circ} 39^{\prime} .3 \mathrm{~N} \\
13^{\circ} 39^{\prime} .3 \mathrm{E}\end{array}$ & V & 10 & 4.4 & $1.6 \times 10^{18}$ \\
\hline 289 & $\begin{array}{c}8-\mathrm{V}-" ~ " \\
19^{\mathrm{h}} 55^{\mathrm{m}} 34^{\mathrm{s}}\end{array}$ & $\begin{array}{c}\text { Montorio al } \\
\text { Vomano }\end{array}$ & $\begin{array}{l}42^{\circ} 34^{\prime} .9 \mathrm{~N} \\
13^{\circ} 37^{\prime} .7 \mathrm{E}\end{array}$ & $\mathrm{V}$ & 15 & 4.6 & $10^{18}$ \\
\hline 290 & $\begin{array}{l}24-V I-" ~ \\
06^{14} 07^{m} 05^{s}\end{array}$ & L'Aquila & $\begin{array}{l}42^{\circ} 20^{\prime} 57^{\prime \prime} \mathrm{N} \\
13^{\circ} 26^{\prime} 26^{\prime \prime} \mathrm{E}\end{array}$ & VIII & 10 & 6.2 & $3.2 \times 10^{21}$ \\
\hline 291 & $\begin{array}{c}1-1-1959 \\
23^{\mathrm{h}} 58^{\mathrm{m}}\end{array}$ & $\begin{array}{c}\text { Torricella } \\
\text { Sicura }\end{array}$ & $\begin{array}{l}42^{\circ} 39^{\prime} .3 \mathrm{~N} \\
13^{\circ} 39^{\prime} .3 \mathrm{~F}\end{array}$ & VI & 11 & 5.0 & $2.5 \times 10^{1 \theta}$ \\
\hline
\end{tabular}




\begin{tabular}{|c|c|c|c|c|c|c|c|}
\hline No & I)ata & Jucalitì & $\begin{array}{c}\text { Coord. Ep. } \\
\varphi \quad \lambda .\end{array}$ & $I_{0}$ & $\begin{array}{c}h \\
\mathrm{~km} m\end{array}$ & Ir & $\begin{array}{c}E \\
\text { ergs }\end{array}$ \\
\hline 292 & $\begin{array}{c}20-11-1959 \\
09^{\mathrm{h}} \pm\end{array}$ & $\begin{array}{c}\text { Campli - Ci- } \\
\text { vitella del } \\
\text { Tronto- } \\
\text { Tagliacozzo }\end{array}$ & $\begin{array}{ll}42^{\circ} 45^{\prime} & \mathrm{N} \\
13^{\circ} 41^{\prime} & \mathrm{F}\end{array}$ & IV & 9 & 3.8 & $10^{17}$ \\
\hline 293 & $\begin{array}{c}1+.111-1960 \\
04^{\mathrm{h}} 45^{\mathrm{m}}\end{array}$ & Cappadocia & $\begin{array}{l}42^{\circ} 02^{\prime} \mathrm{N} \\
13^{\circ} 15^{\prime} .7 \mathrm{E}\end{array}$ & V1I & 5 & 5.3 & $2.5 \times 10^{10}$ \\
\hline 294 & $\begin{array}{c}16-111-" ~ \\
01^{\mathrm{h}} 53^{\mathrm{m}} 05\end{array}$ & $\begin{array}{l}\text { Montemo- } \\
\text { naco-Cit- } \\
\text { tareale }\end{array}$ & - & VI & 20 & 5.3 & $2 \times 10^{20}$ \\
\hline 295 & $\begin{array}{c}29-1-1961 \\
22^{\mathrm{h}} 32.5^{\mathrm{m}}\end{array}$ & Isernia & $\begin{array}{ll}41^{\circ} 36^{\prime} & N \\
14^{\circ} 06^{\prime} & \mathrm{E}\end{array}$ & $V$ & 15 & 4.6 & $10^{19}$ \\
\hline 296 & $\begin{array}{c}30-1-" ~ \\
22^{\mathrm{n}} 30^{\mathrm{m}}\end{array}$ & $\begin{array}{l}\text { Villetta } \\
\text { Barrea }\end{array}$ & $\begin{array}{l}41046^{\prime} .5 \mathrm{~N} \\
13556^{\prime} \mathrm{F}\end{array}$ & IV & 12 & 4.0 & $2.5 \times 10^{17}$ \\
\hline $296 a$ & $\begin{array}{c}25-\text { III- } \\
11^{\mathrm{h}} 40^{\mathrm{m}}\end{array}$ & Pizzoli & - & V & 10 & 4.4 & $2 \times 10^{18}$ \\
\hline 297 & $\begin{array}{l}20-\text { IV } \\
\text { I } 2^{\mathrm{h}} 34^{\mathrm{m}} 42^{\mathrm{s}}\end{array}$ & Capistrello & $\begin{array}{l}41^{\circ} 57^{\prime}, 8 \mathrm{~N} \\
13^{\circ} 23^{\prime}\end{array}$ & VII & 12 & 5.7 & $5 \times 10^{20}$ \\
\hline 298 & $\begin{array}{l}10-I V . \\
06^{\mathrm{h}} 55.9^{\mathrm{m}}\end{array}$ & $\begin{array}{c}\text { Rocea di } \\
\text { Botte - } \\
\text { Arsoli - } \\
\text { Cervara }\end{array}$ & $\begin{array}{ll}42^{\circ} 01^{\prime} & N \\
13^{\circ} 04^{\prime} & \mathrm{E}\end{array}$ & VI & 7 & 4.8 & $5 \times 10^{18}$ \\
\hline 299 & $\frac{12-1 V-" 1 "}{00^{\mathrm{h}}+33^{\mathrm{m}}}$ & $\begin{array}{l}\text { Cervara } \\
\text { Pereto - } \\
\text { Rocca di } \\
\text { Botte }\end{array}$ & $\begin{array}{l}42^{\circ} 02^{\prime} .5 \underset{\mathrm{N}}{\mathrm{N}} \\
13^{\circ} 05^{\prime}\end{array}$ & VI & 7 & 4.8 & $5 \times 10^{18}$ \\
\hline 300 & $\begin{array}{c}12-I V-" ~ \\
01 \mathrm{~h} 30 \mathrm{~m}\end{array}$ & J'Aquila & - & I V & 7 & 3.6 & $5 \times 10^{17}$ \\
\hline 301 & $\begin{array}{l}11-V I-") \\
06^{\mathrm{h}} 46^{\mathrm{m}} 04^{\mathrm{s}}\end{array}$ & $\begin{array}{l}\text { Presso Sor- } \\
\text { genti del } \\
\text { Peschiera }\end{array}$ & $\begin{array}{l}42^{\circ} 21^{\prime} 54^{\prime \prime} \mathrm{N} \\
13^{\circ} 00^{\prime} 28^{\prime \prime} \mathrm{E}\end{array}$ & IV & 5 & 3.5 & $1.6 \times 10^{16}$ \\
\hline 302 & $\begin{array}{l}15-V 1 . " ~ \\
07^{n} 26^{\mathrm{m}} 34^{\mathrm{s}}\end{array}$ & $"$ & " & IV & 5 & 3.5 & $1.6 \times 10^{18}$ \\
\hline 303 & $\begin{array}{l}\text { 4-VIII- " } \\
14^{\text {h }} 30^{\mathrm{m}} 38^{\mathrm{s}}\end{array}$ & $n$ & " " & IV & 1.2 & 2.9 & $2 \times 10^{14}$ \\
\hline 304 & $\begin{array}{l}13 \cdot \mathrm{IX}-" " \\
01^{\mathrm{h}} 05^{\mathrm{m}} 53^{\mathrm{s}}\end{array}$ & $"$ & $"$ & $1 \mathrm{~V}$ & 1.1 & 2.8 & $1.3 \times 10^{14}$ \\
\hline 305 & $\begin{array}{l}5 \cdot X-" ~ \\
20^{\mathrm{h}} 59^{\mathrm{m}} 36^{\mathrm{s}}\end{array}$ & " & $"$ & VI & 5.3 & 3.5 & $3.2 \times 10^{18}$ \\
\hline 306 & $\begin{array}{l}31-X- \\
14^{\mathrm{h}} 37^{\mathrm{m}} 18^{\mathrm{s}} 6\end{array}$ & $"$ & $n$ & VIII & 8 & 6.1 & $1.6 \times 10^{21}$ \\
\hline 307 & $\begin{array}{l}31-X-{ }^{\prime \prime} \\
16^{\mathrm{h}} 12^{\mathrm{m}} 09^{\mathrm{s}}\end{array}$ & $"$ & $"$ & IV & 5 & 3.5 & $1.6 \times 10^{16}$ \\
\hline 308 & $\begin{array}{l}31-X-" ~ \\
16^{\mathrm{h}} 22^{\mathrm{m}} 56^{\mathrm{s}}\end{array}$ & $"$ & $"$ & $1 \mathrm{~V}$ & 5.3 & 3.6 & $1.6 \times 10^{16}$ \\
\hline 309 & 31-X- $\left." 1{ }^{1 \mathrm{~h}} 31 \mathrm{~m}\right] 3^{\mathrm{s}}$ & $"$ & ” & IV & 5.8 & 3.6 & $2.5 \times 10^{16}$ \\
\hline 310 & $\begin{array}{l}31-X-" ~ \\
16^{\mathrm{n}} 37^{\mathrm{m}} 47^{\mathrm{s}}\end{array}$ & " & $"$ & IV & 5 & 3.5 & $1.6 \times 10^{16}$ \\
\hline 311 & $\begin{array}{l}31-X-" \prime \prime \\
17^{1105} 5^{m} 7^{\mathrm{s}}\end{array}$ & $"$ & " $\quad 1$ & IV & 5 & 3.5 & $1.6 \times I 0^{16}$ \\
\hline 312 & $\begin{array}{l}31-\mathrm{X}-" ” \\
17^{\circ} 09^{\mathrm{m}} 33^{\mathrm{s}} .5\end{array}$ & $"$ & $n$ & IV & 5 & 3.5 & $1.6 \times 10^{16}$ \\
\hline
\end{tabular}




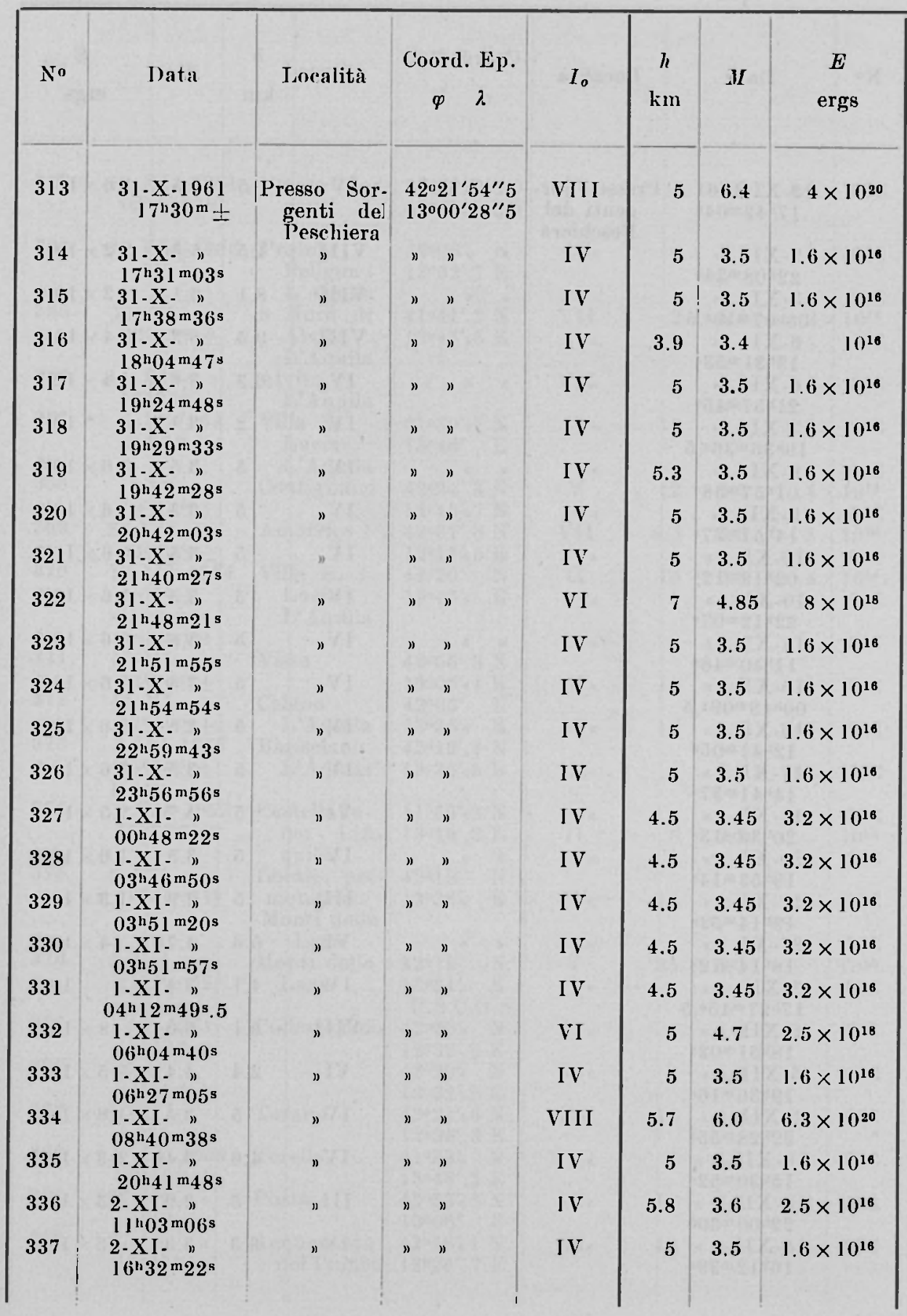




\begin{tabular}{|c|c|c|c|c|c|c|c|}
\hline No & Data & Jocalità & $\begin{array}{c}\text { (Goord. Ep. } \\
\varphi \lambda\end{array}$ & $I_{0}$ & $\begin{array}{c}h \\
\mathrm{~km}\end{array}$ & $M$ & $\begin{array}{c}E \\
\text { ergs }\end{array}$ \\
\hline 338 & $\begin{array}{l}2-\mathrm{XI}-1961 \\
17^{\mathrm{h}} 42^{\mathrm{m} 04^{\mathrm{s}}}\end{array}$ & $\begin{array}{c}\text { Presso Sor- } \\
\text { genti del }\end{array}$ & $\begin{array}{l}42^{\circ} 21^{\prime} 54^{\prime \prime} 5 \\
13^{\circ} 00^{\prime} 28^{\prime \prime} 5\end{array}$ & IV & 5 & 3.5 & $1.6 \times 10^{16}$ \\
\hline 339 & $\begin{array}{l}2-\mathrm{XI}-" \mathrm{n} \\
22^{\mathrm{n}} 08^{\mathrm{m}} 34^{\mathrm{s}}\end{array}$ & ( & " " & VIII & 4.5 & 5.85 & $3.2 \times 10^{20}$ \\
\hline 340 & $\begin{array}{l}\text { 3-XI- " } \\
05^{\text {h } 07^{\mathrm{m}} 19^{\mathrm{B}} .5}\end{array}$ & $"$ & $" n$ & VIII & 8.1 & 6.1 & $2 \times 10^{21}$ \\
\hline 341 & $\begin{array}{l}6-X I-" ~ \\
13^{\mathrm{h}} 31^{\mathrm{m}} 53^{\mathrm{s}}\end{array}$ & ") & $" 1$ & VIII & 9.5 & 6.2 & $4 \times 10^{21}$ \\
\hline 342 & $\begin{array}{l}6-X I \\
21^{\mathrm{h}} 57^{\mathrm{m}} 45^{\mathrm{s}}\end{array}$ & $"$ & $" n$ & IV & 6.3 & 3.6 & $4 \times 10^{16}$ \\
\hline 343 & $\begin{array}{c}7-X I-" ~ \\
19^{\mathrm{h}} 36^{\mathrm{m}} 36^{\mathrm{s}} .5\end{array}$ & ” & $"$ & IV & 2 & 3.1 & $10^{15}$ \\
\hline 344 & $\begin{array}{l}9 \cdot \mathrm{XI}-" ~ \\
01^{\mathrm{h}} 57^{\mathrm{m}} 58^{\mathrm{s}}\end{array}$ & $"$ & $" n$ & IV & 5 & 3.5 & $1.6 \times 10^{16}$ \\
\hline 345 & $\begin{array}{l}9-X I-" \\
14^{\mathrm{h}} 51^{\mathrm{m}} 37^{\mathrm{s}}\end{array}$ & $"$ & 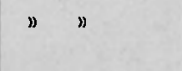 & IV & 5 & 3.5 & $1.6 \times 10^{16}$ \\
\hline 346 & $\begin{array}{c}10-X I-" ~ \\
02^{\mathrm{h}} 19^{\mathrm{m}} 12^{\mathrm{s}}\end{array}$ & $"$ & 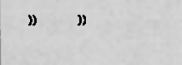 & IV & 5 & 3.5 & $1.6 \times 10^{16}$ \\
\hline 347 & $\begin{array}{l}10-\mathrm{XI}-" \\
22^{\mathrm{n}} 12^{\mathrm{m}} 07^{\mathrm{s}}\end{array}$ & $n$ & $" n$ & IV & 5 & 3.5 & $1.6 \times 10^{16}$ \\
\hline 348 & $\begin{array}{l}14-X I-" ~ \\
11^{\mathrm{h}} 40^{\mathrm{m}} 46^{\mathrm{s}}\end{array}$ & $"$ & $"$ & IV & 5 & 3.5 & $1.6 \times 10^{16}$ \\
\hline 349 & $\begin{array}{l}15-X I-" \\
00^{\mathrm{h}} 48^{\mathrm{m}} 09^{\mathrm{s}} .5\end{array}$ & $"$ & $" \equiv$ & IV & 5 & 3.5 & $1.6 \times 10^{16}$ \\
\hline 350 & $\begin{array}{l}16-\mathrm{XI} \cdot " \\
12^{\mathrm{h}} 41^{\mathrm{m}} 05^{\mathrm{s}}\end{array}$ & $"$ & " & IV & 5 & 3.5 & $1.6 \times 10^{16}$ \\
\hline 351 & $\begin{array}{c}17-X I-" ~ \\
14^{\mathrm{h}} 41^{\mathrm{m}} 3^{\mathrm{s}}\end{array}$ & $n$ & $" \quad$ & IV & 5 & 3.5 & $1.6 \times 10^{16}$ \\
\hline 352 & $\begin{array}{c}17-\mathrm{XI} . " \prime \\
20^{\mathrm{n}} 30^{\mathrm{m}} 13^{\mathrm{s}}\end{array}$ & $"$ & $" n$ & VI & 5 & 4.7 & $2.5 \times 10^{18}$ \\
\hline 353 & $\begin{array}{c}20-X 1-" ~ \\
19^{\mathrm{h}} 53^{\mathrm{m}} 14^{\mathrm{s}}\end{array}$ & $"$ & $"$ & IV & 5 & 3.5 & $1.6 \times 10^{16}$ \\
\hline 354 & $\begin{array}{c}22-X I-" ~ \\
19^{\text {n } 14^{\mathrm{m}}} 5^{\prime}=\end{array}$ & $"$ & $n$ & II I & 5 & 2.9 & $1.3 \times 10^{15}$ \\
\hline 355 & $\begin{array}{l}27-\mathrm{XI}-" \\
18^{\mathrm{h}} 14^{\mathrm{m}} 12^{\mathrm{s}}\end{array}$ & $"$ & $"$ & VI & 5.6 & 4.75 & $4 \times 10^{18}$ \\
\hline 356 & $\begin{array}{l}\text { 3-XII- " } \\
17^{\mathrm{h}} 27^{\mathrm{m}} 45^{\mathrm{s}} .5\end{array}$ & $"$ & $n$ & IV & 4.1 & 3.4 & $10^{16}$ \\
\hline 357 & $\begin{array}{l}5-\mathrm{XII}-" \| \\
18^{\mathrm{h}} 51^{\mathrm{m}} 02^{\mathrm{s}}\end{array}$ & $"$ & $" n$ & V111 & 6.1 & 6.0 & $8 \times 10^{20}$ \\
\hline 358 & $\begin{array}{l}\text { 5.XII. " } \\
19^{\mathrm{h}} 36^{\mathrm{m}} 16^{\mathrm{s}}\end{array}$ & $"$ & " $"$ & VI & 2.4 & 4.4 & $2.5 \times 10^{17}$ \\
\hline 359 & $\begin{array}{l}5 \text {-XII- " } \\
22^{\mathrm{h}} 28^{\mathrm{m}} 55^{\mathrm{s}}\end{array}$ & $n$ & $" n$ & IV & 5 & 3.5 & $1.6 \times 10^{16}$ \\
\hline 360 & $\begin{array}{c}10-\text { XII } \\
15^{\mathrm{h}} 30^{\mathrm{m}} 5^{\prime \prime}\end{array}$ & $"$ & $" n$ & IV & 4.6 & 3.46 & $1.3 \times 10^{16}$ \\
\hline 361 & $\begin{array}{l}10 \text {-XII } \\
22^{\mathrm{h}} 00^{\mathrm{m}} 30^{\mathrm{s}}\end{array}$ & $"$ & $n \quad n$ & III & 5 & 2.9 & $1.3 \times 10^{15}$ \\
\hline 362 & $\begin{array}{l}11-\text { XII - " } \\
16^{\mathrm{h}} 12^{\mathrm{m}} 39^{\mathrm{s}}\end{array}$ & $"$ & $" n$ & IV & 3.3 & 3.3 & $5 \times 10^{15}$ \\
\hline
\end{tabular}




\begin{tabular}{|c|c|c|c|c|c|c|c|}
\hline $\mathrm{N}^{\circ}$ & Data & Località & $\begin{array}{c}\text { Coord. Ep. } \\
\varphi \lambda\end{array}$ & $I_{0}$ & $\begin{array}{r}h \\
\mathrm{~km}\end{array}$ &.$I I$ & $\begin{array}{c}E \\
\text { ergs }\end{array}$ \\
\hline 363 & $\begin{array}{c}19-X I[-1961 \\
02^{\mathrm{h}} 16^{\mathrm{m}} 19^{\mathrm{s}}\end{array}$ & $\begin{array}{c}\text { Presso Sor- } \\
\text { genti del } \\
\text { Peschiera }\end{array}$ & $\begin{array}{l}42^{\circ} 21^{\prime} 54^{\prime \prime} .5 \\
13^{\circ} 00^{\prime} 28^{\prime \prime} .5\end{array}$ & I V & 5 & 3.5 & $1.6 \times 10^{16}$ \\
\hline 364 & $\begin{array}{c}\text { 30.VII-1962 } \\
11^{\mathrm{h}} \pm\end{array}$ & $\begin{array}{l}\text { Pratola } \\
\text { Peligna - } \\
\text { L'Aquila }\end{array}$ & $\begin{array}{ll}42^{\circ} 06^{\prime} & \mathrm{N} \\
13^{\circ} 52^{\prime} .7 \mathrm{E}\end{array}$ & IV & 8 & 3.7 & $8 \times 10^{18}$ \\
\hline 365 & $\begin{array}{c}21-\mathrm{XI}-" ~ \\
22^{\mathrm{h}} 20^{\mathrm{m}} \pm\end{array}$ & $\begin{array}{l}\text { a Nord di } \\
\text { Alvito - } \\
\text { L'Aquila }\end{array}$ & $\begin{array}{l}41^{\circ} 41^{\prime} .2 \mathrm{~N} \\
13^{0} 44^{\prime} .6 \mathrm{l}\end{array}$ & VII & 2 & 4.9 & $2 \times 10^{18}$ \\
\hline 366 & $\begin{array}{c}14-X I I-" ~ \\
13^{\mathrm{h}} 35^{\mathrm{m}} \pm\end{array}$ & $\begin{array}{l}\text { Alvito } \\
\text { L'Aquila }\end{array}$ & $" n$ & VII & 4 & 5.2 & $1.6 \times 10^{18}$ \\
\hline 367 & $\begin{array}{l}5-I I-19 \overline{63} \\
8^{\mathrm{h}} 30^{\mathrm{m}} \pm\end{array}$ & $\begin{array}{l}\text { Villa S. } \\
\text { Lucia } \\
\text { L'Aquila }\end{array}$ & $\begin{array}{l}41^{\circ} 30^{\prime} .7 \mathrm{~N} \\
13^{\circ} 46^{\prime}\end{array}$ & I V & 15 & 4.0 & $6.3 \times 10^{17}$ \\
\hline 368 & $\begin{array}{c}5 \text {-II - " } \\
19^{\mathrm{h}} 15^{\mathrm{m}} \pm\end{array}$ & Contigliano & $\begin{array}{l}42^{\circ} 24^{\prime} .3 \mathrm{~N} \\
12^{\circ} 45^{\prime} .7 \mathrm{E}\end{array}$ & V & 12 & 4.5 & $4 \times 10^{18}$ \\
\hline 369 & $\begin{array}{l}\text { 24-VII- " } \\
03^{\mathrm{h}} 40^{\mathrm{m}} \pm\end{array}$ & Amatrice & $\begin{array}{l}42^{\circ} 37^{\prime} .6 \mathrm{~N} \\
13^{\circ} 17^{\prime} .5 \mathrm{E}\end{array}$ & VII & 8.5 & 5.5 & $2 \times 10^{20}$ \\
\hline 370 & $\begin{array}{c}17-I I I-1 \frac{1}{965} \\
02^{\mathrm{h}} \pm \\
\text { scossa pre- } \\
\text { monitrice }\end{array}$ & $\begin{array}{l}\text { Villa S. } \\
\text { Lucia- } \\
\text { L'Aquila }\end{array}$ & $\begin{array}{ll}42^{\circ} 20^{\prime} & \mathrm{N} \\
13^{\circ} 47^{\prime} & \mathrm{E}\end{array}$ & II & 15 & 2.8 & $4 \times 10^{15}$ \\
\hline 371 & $\begin{array}{c}\text { 17-III. " } \\
02^{\mathrm{h}} 30^{\mathrm{m}} \pm\end{array}$ & Visso & $\begin{array}{l}42^{\circ} 55^{\prime} .8 \mathrm{~N} \\
13^{\circ} 05^{\prime} .1 \mathrm{E}\end{array}$ & V & 18 & 4.7 & $1.3 \times 10^{19}$ \\
\hline 372 & $\begin{array}{c}12-\mathrm{XI}-" ~ \\
07^{\mathrm{h}} 20^{\mathrm{m}} \pm\end{array}$ & Celano - & $\begin{array}{ll}42^{\circ} 05^{\prime} & \mathrm{N} \\
13^{\circ} 36^{\prime} & \mathrm{E}\end{array}$ & IV & 10 & 3.8 & $1.6 \times 10^{17}$ \\
\hline 373 & $\begin{array}{l}3-I I I-1960 \\
05^{\mathrm{h}} 10^{\mathrm{m}} \pm \\
\text { locale }\end{array}$ & $\begin{array}{l}\text { Barisciano - } \\
\text { L'Aquila }\end{array}$ & $\begin{array}{l}42^{\circ} 19^{\prime} .4 \mathrm{~N} \\
13^{\circ} 35^{\prime} .5 \mathrm{E}\end{array}$ & I I I & 5 & 2.9 & $1.3 \times 10^{15}$ \\
\hline 374 & $\begin{array}{c}3-X I I-1967 \\
17439 \mathrm{~m} 10^{8}\end{array}$ & $\begin{array}{l}\text { Castellafiu- } \\
\text { me - L'A- } \\
\text { quila }\end{array}$ & $\begin{array}{l}41^{\circ} 59^{\prime} .2 \mathrm{~N} \\
13^{\circ} 19^{\prime} .2 \mathrm{E}\end{array}$ & II & 5 & 2.3 & $10^{14}$ \\
\hline 375 & $\begin{array}{l}\text { 3.XII- " } \\
19^{\mathrm{h}} 49^{\mathrm{m}} 4 \mathrm{l}^{\mathrm{s}}\end{array}$ & $\begin{array}{l}\text { Locale, pre- } \\
\text { monitrice. } \\
\text { Monti della } \\
\text { Laga }\end{array}$ & $\begin{array}{ll}42^{\circ} 18^{\prime} & \mathrm{N} \\
13^{\circ} 36^{\prime} & \mathrm{E}\end{array}$ & IV & 18 & 4.1 & $10^{18}$ \\
\hline 376 & $\begin{array}{l}\text { 3-XII- " } \\
21^{\text {h }} 29^{\mathrm{m}} 59^{\mathrm{s}}\end{array}$ & $\begin{array}{l}\text { Monti della } \\
\text { Laga }\end{array}$ & $\begin{array}{cc}42^{\circ} 18^{\prime} & \mathrm{N} \\
13^{\circ} 24^{\prime} & \mathrm{E} \\
\text { U.S.C.G.S. }\end{array}$ & $\mathrm{V}$ & 35 & 4.7 & $10^{20}$ \\
\hline 377 & $\begin{array}{r}1-I-1968 \\
10^{\mathrm{h}} 20^{\mathrm{m}} \pm\end{array}$ & Collevecchio & $\begin{array}{l}42^{\circ} 20^{\prime} \mathrm{N} \\
12^{\circ} 32^{\prime} .9 \mathrm{E}\end{array}$ & VI & 5 & 4.1 & $2.5 \times 10^{18}$ \\
\hline 378 & $\begin{array}{c}\text { 5-I- } \\
06^{\mathrm{h}} 05^{\mathrm{m}} \pm\end{array}$ & $"$ & $\begin{array}{l}42^{\circ} 20^{\prime} \quad \mathrm{N} \\
12^{\circ} 32^{\prime} .9 \mathrm{E}\end{array}$ & VI & 6 & 4.2 & $5 \times 10^{18}$ \\
\hline 379 & $\begin{array}{c}8 \cdot \text { VIII- } \\
05^{\mathrm{h}} \pm "\end{array}$ & Tarano & $\begin{array}{l}42^{\circ} 21^{\prime} .5 \mathrm{~N} \\
12^{\circ} 35^{\prime} .3 \mathrm{E}\end{array}$ & 111 & 15 & 3.4 & $5 \times 10^{16}$ \\
\hline 380 & $\begin{array}{c}17 . I V .1969 \\
09^{\mathrm{h}} 10^{\mathrm{m}} \pm\end{array}$ & Terelle & $\begin{array}{l}41^{\circ} 33^{\prime} \quad \mathrm{N} \\
13^{\circ} 46^{\prime} .3 \mathrm{E}\end{array}$ & VIII & 3 & 5.7 & $8 \times 10^{19}$ \\
\hline 381 & $\begin{array}{c}27-I X . \\
09^{h} \pm "\end{array}$ & Posta & $\begin{array}{l}42^{\circ} 33^{\prime}, 5 \mathrm{~N} \\
13^{\circ} 06^{\prime}\end{array}$ & IV & 10 & 3.8 & $1.6 \times 10^{17}$ \\
\hline 382 & $\begin{array}{c}27-\mathrm{IX}-" \\
12^{\mathrm{h}} 45^{\mathrm{m}} \pm\end{array}$ & $\begin{array}{l}\text { Acquasanta } \\
\text { del Tronto }\end{array}$ & $\begin{array}{l}42^{\circ} 46^{\prime} .1 \mathrm{~N} \\
13^{\circ} 24^{\prime} .7 \mathrm{E}\end{array}$ & VI & 13 & 4.5 & $5.5 \times 10^{19}$ \\
\hline
\end{tabular}


III. - LA SISMICITA IN RELAZIONE CON LA TETTONICA REGIONALE.

La teoria sulla traslazione dell'Appennino laziale-abruzzese non sembra contraddire, nelle grandi linee, alle ipotesi più volte avanzate da Caloi e collaboratori sui movimenti di dette masse litoidi.

L'esame sismo-tettonico di detta regione ha constatato, attraverso le ricostruzioni già illustrate dalle interazioni caratteristiche fra zona

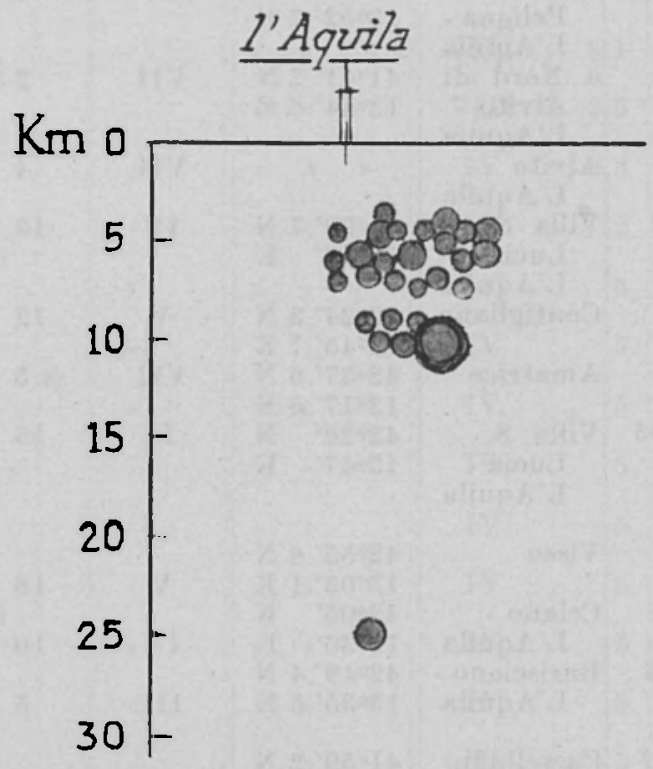

Fig. 2 - Scosse nella zona de L'Aquila avvenute nel 1900-1902, 1904-1907, 1929, 1938-1941, 1958. Magnitudo: $3<M \leqslant 6.2$

(I numeri in ordinata esprimono la profondità ipocentrale approssimativa).

epicentrale sismica e frattura tettonica, come esista una relazione, come da causa ad effetto o ancora come da effetto a causa, fra i terremoti recenti e gli sconvolgimenti più o meno vistosi che si sono prodotti e, che in scala minore, continuano a manifestarsi. Poiché a fenomeni sismici superficiali corrispondono all'ipocentro modalità altrettanto caratteristiche e specifiche, possiamo senz'altro considerare brevemente, alla luce di dati sperimentali, in un panorama che investe tutte le forme tipiche sismiche che hanno interessato e interessano la nostra Penisola, soffermandoci solo dove la materia del nostro studio diventa specifica, le relazioni che legano la sismicità all'orogenesi, in modo da avere gli elementi sufficienti per avvalorare la nostra dissertazione. 
Dalla direzione del movimento determinato in superficie dalle onde longitudinali $P_{g}$ e $P_{n}$ nelle stazioni d'osservazione, è possibile dedurre di che natura è stata la scossa all'ipocentro. Tale metodo d'indagine ci ha condotto alla conclusione che le scosse possono essere dovute a fratture, a sollevamenti o abbassamenti di strati. Ricordiamo che, dal punto di vista del senso del movimento provocato, un'onda longitudinale puó essere di dilatazione o di compressione: al primo caso cor-

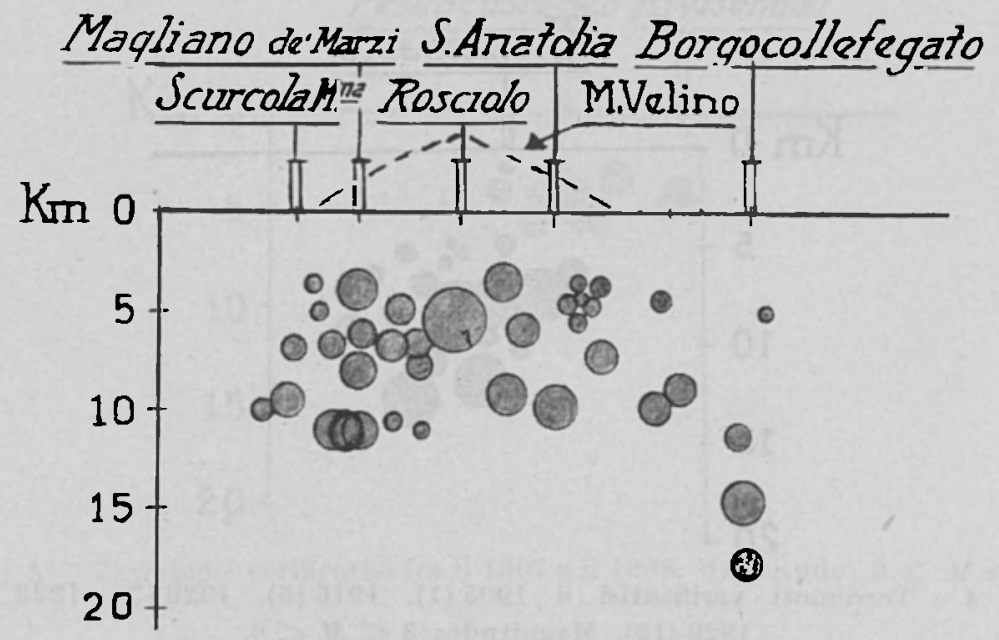

Fig. 3 - Scosso del 1904 nella zona del Velino (S. Anatolia nel 1941). Magnitudo: $4<M \leqslant 6.6$

risponde nella stazione d'osservazione un avvallamento del suolo, al secondo caso un innalzamento.

Dall'osservazione delle tre componenti del movimento - due orizzontali a $90^{\circ}$ fra loro ed una verticale - si puó stabilire a quale dei due casi l'onda appartiene. In occasione di terremoti dovuti a fratture, in certe stazioni e per le stesse distanze epicentrali, si registra in superficie un movimento del suolo nella direzione epicentro-stazione (compressione); in altre il movimento in senso opposto (dilatazione). Nel caso più semplice, se la frattura si verifica su un piano verticale ed è dovuta ad una coppia di forze tangenziali orizzontali, questi movimenti di dilatazione e di compressione si dividono in modo da distribuirsi nei quattro quadranti determinati da due rette perpendicolari, incrociantisi all'epicentro, ed in maniera che nelle due coppie di quadranti opposti si verificano movimenti del suolo dello stesso se- 
gno (compressioni o dilatazioni), mentre in quadranti adiacenti i movimenti sono di segno opposto. Per rappresentare, sia pure in maniera elementare, il modo con cui il fenomeno si verifica dal punto di vista dell'elasticità, diremo che queste fratture sono dovute all'azione di coppie di forze tangenziali che agiscono all'ipocentro. Dai fondamenti della teoria dell'elasticità sappiamo che ad una tensione che agisce secondo un determinato asse, corrisponde sempre una tensione com-

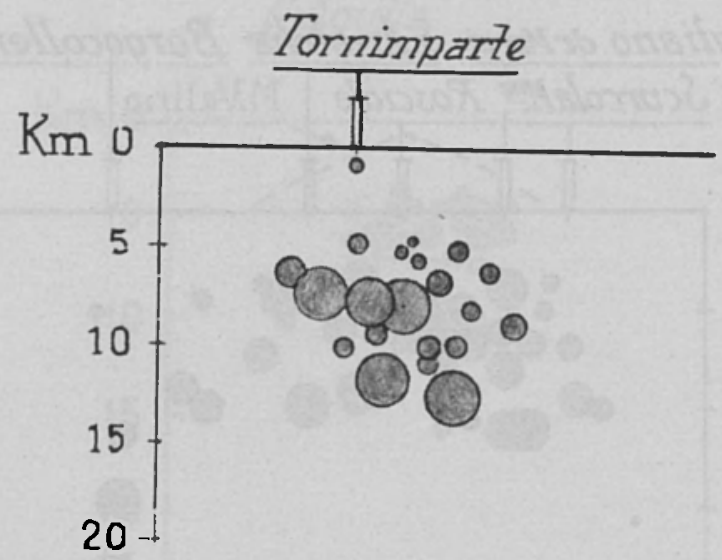

Fig. 4 - Terremoti verificatisi il $1905(1), 1916(5), 1920(3), 1923$ (1), 1929 (12). Magnitudo: $3 \leqslant M \leqslant 6$.

plementare secondo l'asse a $90^{\circ}$, di entità uguale alla prima ma di segno contrario. Analogamente, alla coppia che diremo principale, a cui si deve la frattura degli strati, corrisponde una coppia complementare, che agisce in direzione normale alla prima. In tal modo si viene a spiegare la suddivisione, nel senso del movimento, nei quattro quadranti suddetti.

Nel caso di terremoti dovuti a sprofondamento o sollevamento, in tutte le stazioni comprese in una zona di determinato raggio e in tutti gli azimut, si verifica invece un primo movimento dello stesso segno (dilatazioni o compressioni), e per tutte le stazioni esterne, un movimento di segno opposto (compressioni o dilatazioni).

Sotto il punto di vista della profondità dei terremoti, dobbiamo innanzitutto chiarire che $\mathrm{i}$ terremoti - oggetto del nostro studio appartengono per la maggior parte a quei fenomeni che hanno interessato gli strati superficiali della crosta terrestre, se si eccettuano quelli rappresentanti la sismicità del basso Tirreno che hanno inte- 
ressato le parti profonde (come ad esempio il terremoto del 13 Aprile 1938 delle isole Lipari, avvenuto ad una profondità di circa $300 \mathrm{~km}$ ).

Tutti gli esempi sopra illustrati nella classificazione dei terremoti, hanno corrispettivi pratici nei terremoti che hanno interessato la nostra penisola.

La frattura, con il mutuo scorrimento di strati, è stata osservata in tutto il sistema alpino, dove essa sembra predominare: l'esempio

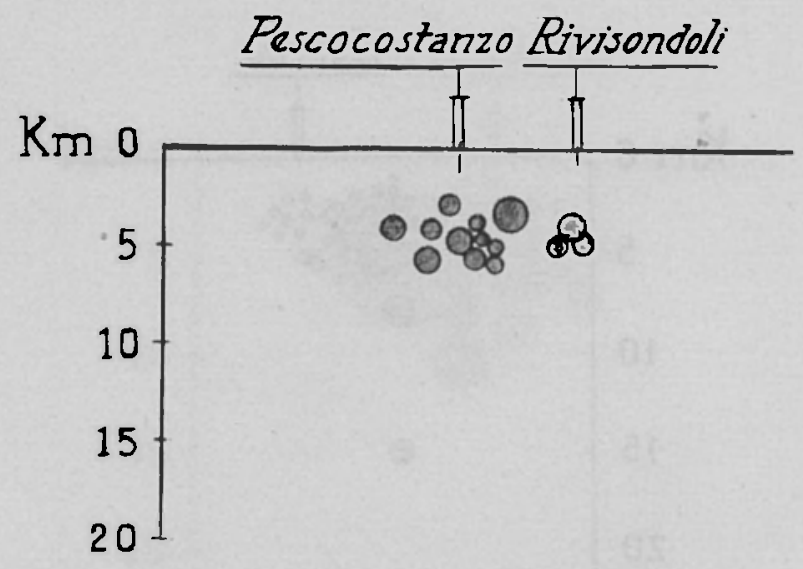

Fig. 5 - 'Terremoti verificatisi fra il 1907 e il 1908. Magnitudo: $3 \leqslant M \leqslant 5.3$

più caratteristico è stato fornito dal terremoto del Cansiglio del 18 Ottobre 1936, dovuto a frattura provocata da una coppia di forze inclinate, giacenti in un piano pure inclinato.

A proposito della profondità ipocentrale, va osservato che $\mathrm{i}$ terremoti interessanti il sistema alpino, in senso lato, riguardano esclusivamente la crosta terrestre; molti fra essi originano a piccole profondità ipocentrali, da un minimo di $10 \mathrm{~km}$ ad un massimo di $20 \mathrm{~km}$.

I terremoti dell'alto Adriatico presentano invece il modello caratteristico dei terremoti di sprofondamento e sembrano originare ai limiti della crosta terrestre. A questo riguardo è interessante osservare il parallelo nei fenomeni bradisismici della regione. E noto infatti che, in ere geologiche pussate, fu molto accentuato il bradisismo negativo in tutta l'Istria e la Dalmazia. Dell'entità di questo bradisismo si hanno chiare testimonianze nella forma frastagliatissima della costa adriatica orientale e nel gran numero di isole da cui è orlata.

Nel Tirreno meridionale i terremoti rientrano nel modello caratteristico delle fratture; qui il fenomeno presenta un interesse a sè, 
trattandosi di terremoti di origine profonda, come quello già citato a pag. 345 delle isole Lipari del 13 Aprile 1938.

In effetti la zona si presenta anomala da tutti i punti di vista: geologico, geodetico, sismico, essendo stata spesso teatro di eruzioni vulcaniche sottomarine, rivelate da terremoti e da maremoti.

L'Appennino presenta invece il fenomeno contrario dell'alto Adriatico. Lo studio dei terremoti dell'Appennino tosco-romagnolo, della

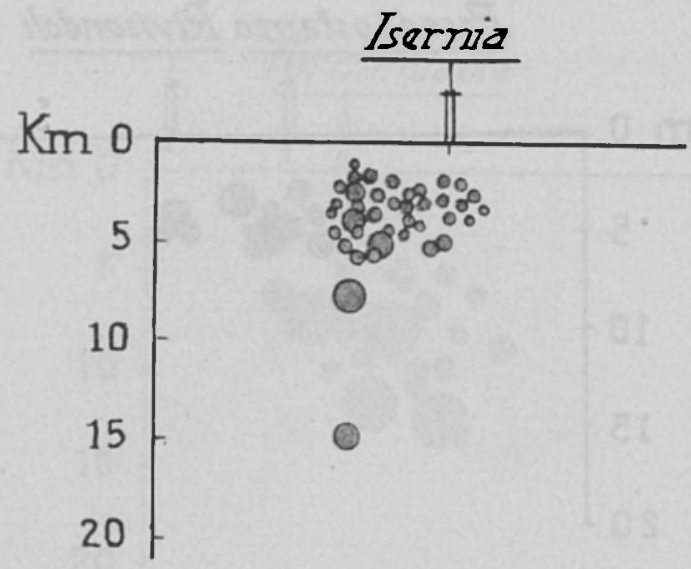

Fig. 6 - Terremoti avvenuti nel 1914 (2), 1917 (2), 1919 (23), 1926 (2), 1932 (6) e 1934 (1). Magnitudo: $3<. I \leqslant 5.5$

Garfagnana, del Monte Amiata, del Gran Sasso d'Italia, dell'Aterno e delle Sorgenti del Peschiera, ha messo chiaramente in luce il carattere di sollevamento di strati. Dal punto di vista geologico, questi sollevamenti trovano testimonianze dall'esame delle fronti di alcune faglie, quali ad esempio quello del Sirente e quello della catena del Gran Sasso verso Campo Imperatore. Le scosse che avvengono nell'ambito degli Appennini originano, come abbiamo visto, quasi esclusivamente a piccola profondità, ma non mancano esempi di profondità superiori ai $20 \mathrm{~km}$.

E di estremo interesse a questo punto, dare il dovuto risalto al particolare fenomeno che distingue la sismicità dell'Adriatico da quella della catena, ad esso parallela, degli Appennini: agli sprofondamenti in profondità dell'Alto Adriatico, fanno riscontro i sollevamenti superficiali della catena appenninica. Alle compressioni laterali determinate dai primi, conseguono i sollevamenti dei secondi. 
Questa fenomenologia, a nostro giudizio, può essere ben valida per spiegare come siano originate forze tali da giustificare il movimento traslativo del complesso laziale-abruzzese. Tra l'altro, il meccanismo con cui si sono originate dette spinte ci sembra anche rispondente alle considerazioni della teoria dell'Isostasia; per cui alla grande forza applicata in profondità, abbia corrisposto in superficie un movimento tendente all'innalzamento e allo scorrimento delle masse in questione.

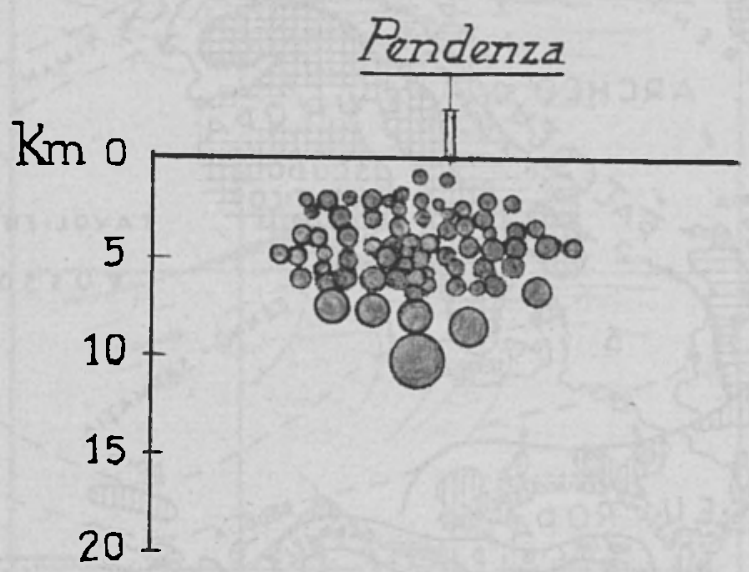

Fig. 7 - Ciclo scosse presso Sorgenti del Peschiera, verificatosi nel 1961. Complessive 63 scosse, di cui 6 con magnitudo dell'ordine di 6. Magnitudo:

$$
3<M \leqslant 6.4
$$

Questi movimenti sono stati resi ancora più vistosi per la presenza di una massa rigida, rappresentata dalla Tirrenide, che ha agito da contrafforte alle sollecitazioni dinamiche, per cui i terreni dello Schelf hanno subíto un vero strizzamento verso la parte orientale dell'Adriatico. La fine del parossismo trova le terre costituenti la Tirrenide frantumate ed immerse nel Tirreno.

A questo iniziale parossismo ne seguirono degli altri, e possiamo dire che sussistono tuttora, ma sia per l'entità minore del fenomeno sia per la mancanza dello scudo rigido della Tirrenide, le componenti delle forze risultanti sono pressoché verticali, per cui abbiamo un effetto in superficie che si riduce ad un innalzamento del terreno.

Fin qui il confronto fra le recentissime indagini geologico-tettoniche e le ricerche sismologiche. A nostro parere, un confronto più significativo, ai fini dell'interpretazione dei moti-origine all'ipocentro, si può istituire fra risultati di ricerche sismologiche e meno recenti studi in 
campo geologico, condotti da Demangeot, Segre, ecc. sotto la guida del compianto Prof. Ramiro Fabiani.

Per quanto concerne le ricerche sismologiche riguardanti l'Abruzzo, e zone limitrofe, si è già detto in precedenza. Esse sono riassunte,

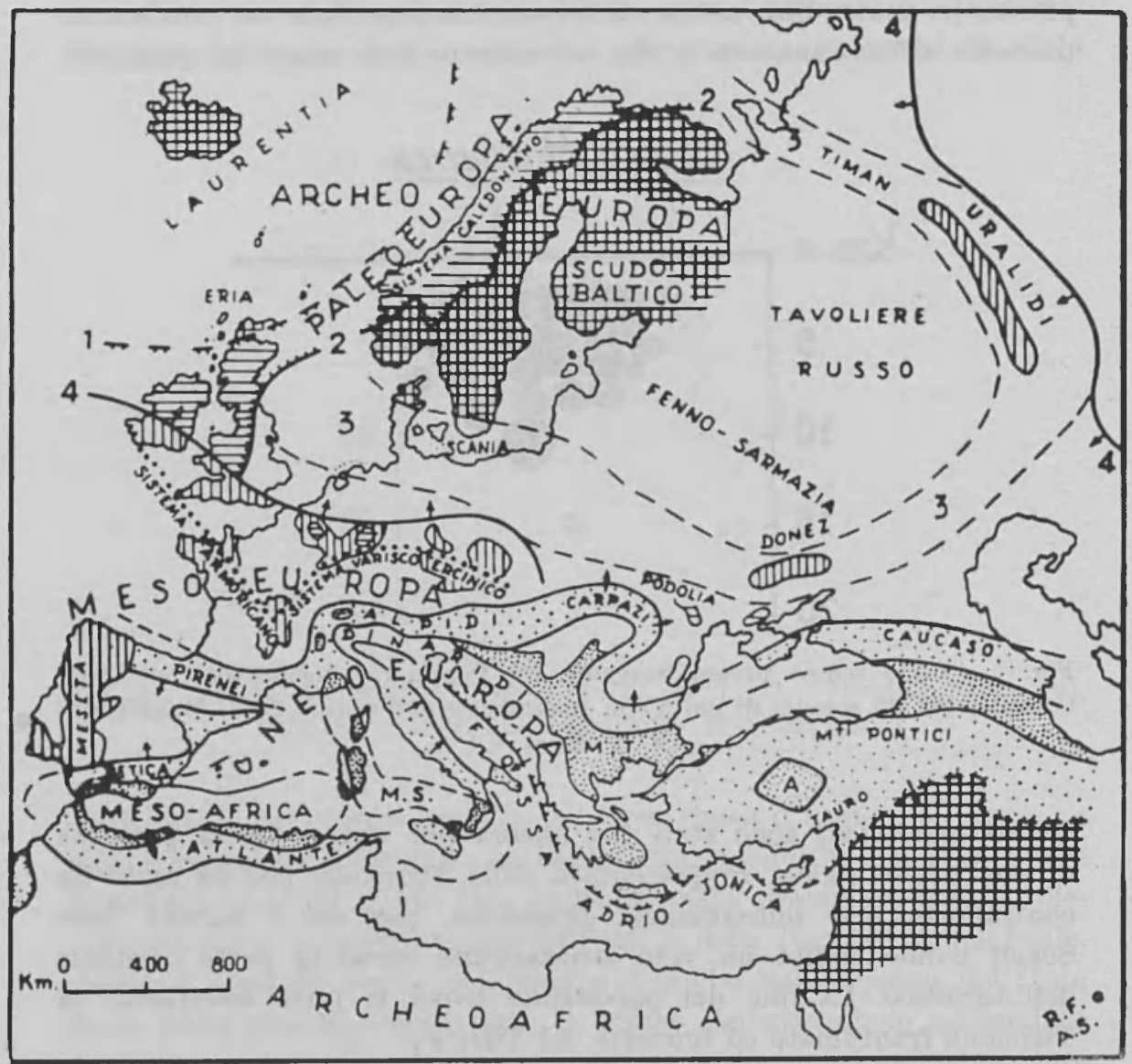

Fig. 8 - L'Italia peninsulare e la fossa adriatica, nel complesso delle zone di corrugamento in Furopa (secondo Fabiani e Segre).

per quanto attiene al meccanismo dei terremoti all'ipocentro, nella Fig. 10. Da essa riesce chiaro il senso dei movimenti improvvisi nella parte esterna della Terra, per la zona che ci interessa: sprofondamenti in corrispondenza della fossa adriatica e sollevamenti nei confronti dell'Appennino centrale. Fa sottolineato che, mentre i primi appaiono 
verificarsi alla base della crosta terrestre, i secondi si presentano con profondità nettamente inferiori, nella generalità essendo tutti contenuti nello strato esterno (" granito ").

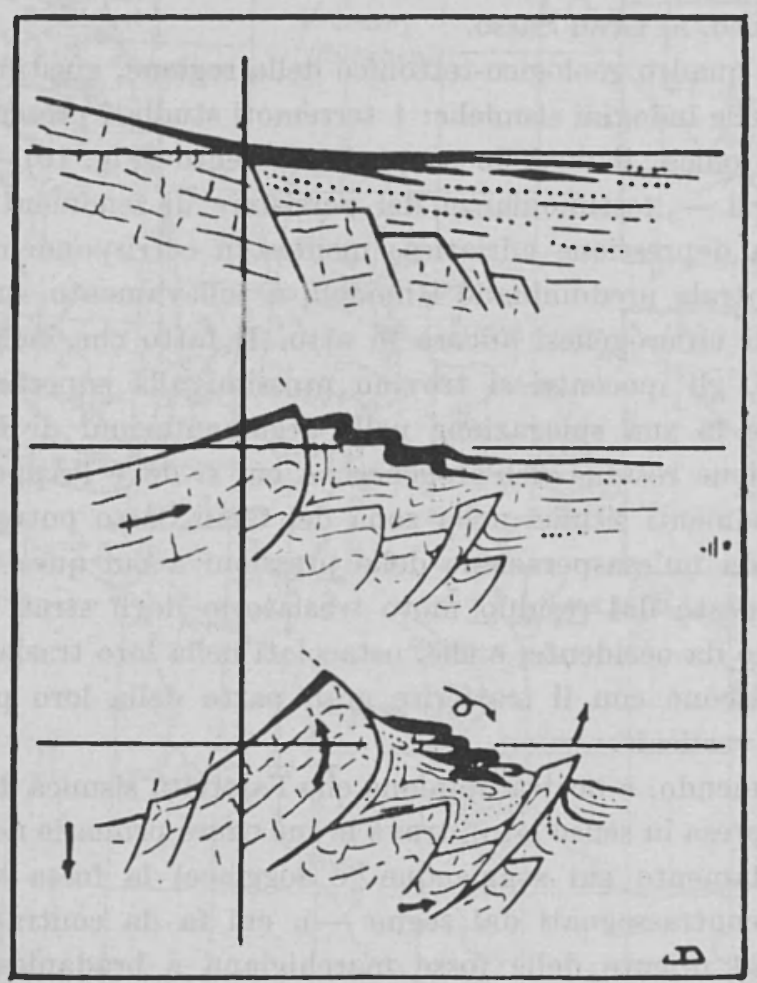

Fig. 9 - Evoluzione del bordo orientale del Gran Sasso. In allo: in nero, i conglomerati mio-pliocenici sparsi dai torrenti sul pianoro calcareo emerso e sulla riva del mare della molassa, verosimilmente in conseguenza di uno strappo tettonico. Nel mezzo: costretto da una spinta laterale, il blocco calcareo solleva la sua hase nascosta. I depositi torrentizi sono infranti e cominciano a scivolare sulla scarpata molassica. In basso: il movimento in compressione si accentua, una scaglia rigila (la Queglia) perfora la molassa, i conglomerati continuano a corrugarsi in disordine (M. Bertona, ecc.), la base occidentale del bloceo sprofonda per distensione (vallata del Tirino) (Secondo Demangeot). Questo meccanismo spiega la natura fisica delle scosse regionali (spinta verso l'esterno; modello hipolare).

Vediamo ora le sintesi geologiche di Fabiani, Demangeot, Segre, ecc. esposti nei " ('ontributi di Scienze Geologiche" del Consiglio Nazionale delle Ricerche (Vol. I - 1950; Vol. II - 1952: anni $20^{\circ}$ e $22^{\circ}$ 
rispettivamente de "La Ricerca Scientifica "). La Fig. 8 dà una visione complessiva delle zone di corrugamento in Europa, secondo concetti generalmente accettati, nella quale quella che a noi interessa (la zona abruzzese-adriatica) entra come particolare, la Fig. 9 si riferisce, in modo specifico, al Gran Sasso.

Questo quadro geologico-tettonico della regione, giustifica in pieno i risultati delle indagini sismiche: $\mathrm{i}$ terremoti studiati presentano, nella fossa adrio-jonica, il modello a sprofondamento (Fig. 10) - come era da attendersi - testimonianza del persistere di fenomeni di affossamento nella depressione adriatica; mentre in corrispondenza dell'Appennino centrale predominano i modelli a sollevamento, anche questi a riprova di un'orogenesi ancora in atto. Il fatto che, nella zona del Gran Sasso, gli ipocentri si trovino prossimi alla superficie esterna, può trovare la sua spiegazione nelle argomentazioni di Demangeot. Oltre all'azione residua dell'orogenesi, a cui si deve l'Appennino centrale, i movimenti sismici nella zona del Gran Sasso potrebbero pure conseguire da un'esasperazione delle pressioni a cui questo massiccio viene sottoposto dal residuo moto traslatorio degli strati superficiali che premono da occidente, e che, ostacolati nella loro traslazione verso oriente, finiscono con il trasferire gran parte della loro pressione in una spinta verticale.

Riassumendo, è nostra opinione che l'attività sismica della regione abruzzese (presa in senso lato), trova le sue cause primarie nel fenomeno di sprofondamento cui soggiacque (e soggiace) la fossa adrio-jonica (terremoti contrassegnati dal segno -), cui fa da contrasto il sollevamento, ad oriente delle fosse marchigiana e bradanica, associato alla formazione degli Appennini (terremoti contrassegnati dal segno +). Questo sollevamento è stato (ed è) agevolato dalla trasformazione in senso verticale della spinta orizzontale proveniente da Ovest e da Sud-Ovest.

E quanto, con altro linguaggio, si deduce dalle citate Figg. 8, 9, e quanto, del resto, è detto nella prima parte del nostro lavoro. Si noti che la traslazione Ovest-Est - oggi attribuita ad una compiacente Tirrenide - avrebbe provocato, nelle zone tirreniche (Circeo ed altre) moti traslativi dell'ordine di alcune diecine di $\mathrm{km}$ (addirittura una cinquantina).

L'entità di dette traslazioni è andata gradatamente scemando a mano a mano che interessava strutture verso l'Appennino centrale, sempre più ostacolate dall'antemurale appenninica (Maiella-Gran Sasso), tanto che le strutture fra il Sirente e il Marsicano non appaiono tra- 
SISMICIT IN RELAZIONE ALLA TETTONICA E ACCUMULO DELLE TENSION] 351

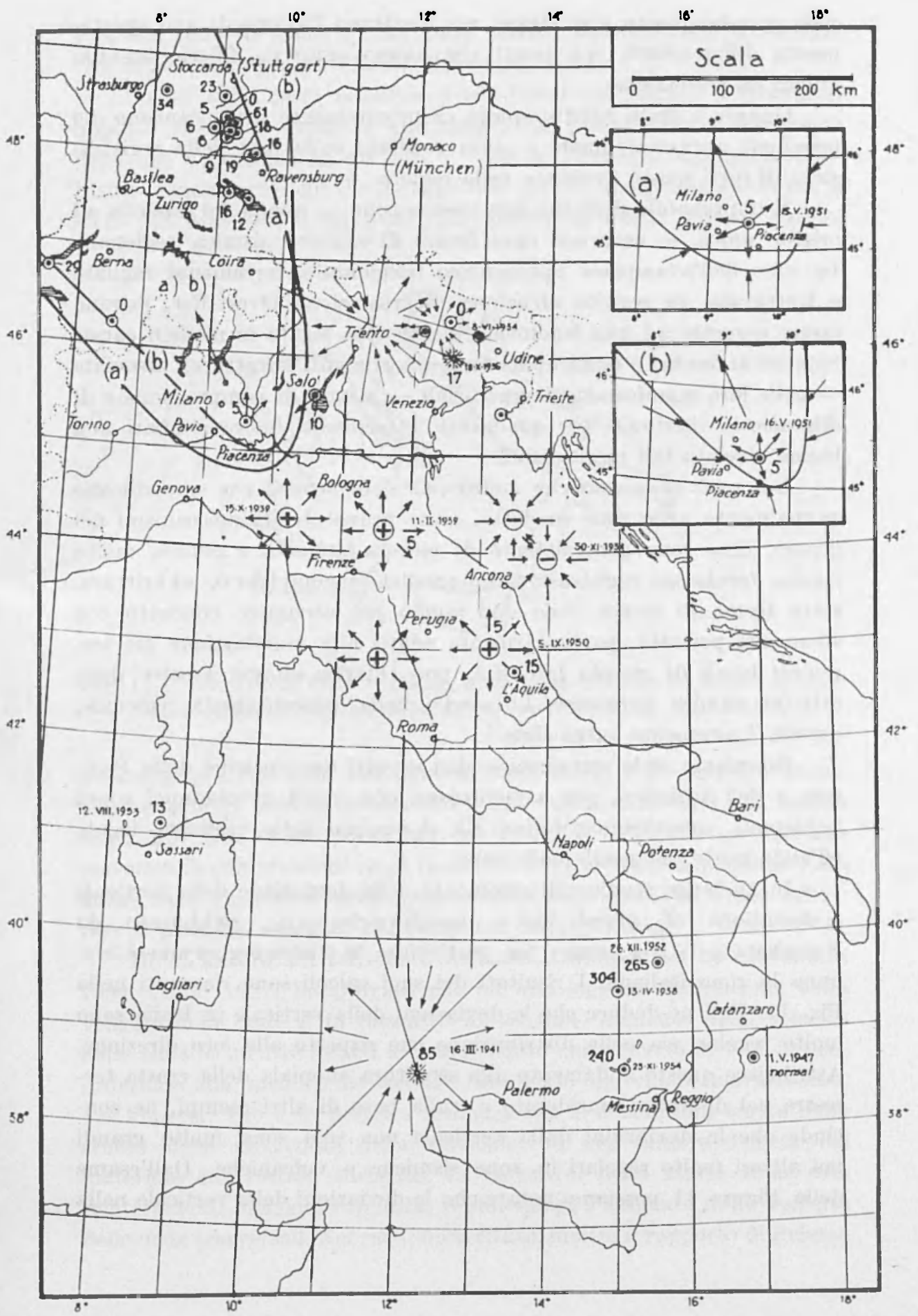

Fig. 10 - Posizione geografica e meccanismo di alcuni terremoti studiati in Italia dopo il 1933. La cifra in grassetto indica la profondita ipocentrale (Secondo Caloi). 
slate parallelamente a se stesse, ma mostrano lazione di uno spostamento differenziato, con punti che hauno agito da fulcro, immuni quindi da traslazione.

Quanto ì stato riferito spiega esaurientemente il meccanismo dei terremoti abruzzesi, quale ̀̀ stato studiato sulle basi delle registrazioni di forti scosse avvenute nella regione.

E improbabile però che tale meccanismo - quello del modello ad origine unica - esaurisca ogni forma di attività sismica regionale. Le zone dell'avampaese appenninico occidentale, fortemente fagliate e fratturate, in seguito all'azione di traslazione Ovest-Est, devono essere soggette ad una fenomenologia sismica legata ai modelli caratteristici di duplici o quadruplici forze (scorrimenti o fratture), associate - nelle loro manifestazioni superficiali - a moti di compressione e di dilatazione distribuiti per quadranti. I terremoti finora studiati non hamno rivelato tali meccanismi.

Noi però riteniamo che moltissime delle scosse, che si verificano annualmente nelle zone su dette, siano provocate da mecanismi del genere. Esse sono generalmente di piccola intensità e quindi, molto spesso, forniscono registrazioni simografiche incomplete o, addirittura, sotto forma di tracce. Solo uno studio più accurato, condotto con strumenti portatili, particolarmente adatti alla registrazione dei terremoti locali di piccola intensità, può chiarire questo aspetto della attività sismica abruzzese. Lo studio della microsismicità regionale, quindi, è altamente auspicabile.

Riteniamo utile sottolineare altri aspetti caratteristici della Penisola e dell'Adriatico, che armonizzano con quelli geodinamici sopra richiamati: intendiamo riferirci alle deviazioni della verticale, legate all'andamento del geoide nella zona.

In un lungo studio sull'argomento della deviazione della verticale (" deviations of plumb-line ", Lotabweichungen), pubblicato da Kawabata nel $195 \mathrm{t}$, come caso particolare lo studioso giapponese esamina la zona italiana. I risultati dei suoi calcoli sono riassunti nella Fig. 11. Egli ne deduce che le deviazioni della verticale in Italia sono molto regolari sia nella distribuzione che rispetto alla loro direzione. Attribuisce questo andamento alla struttura anomala della crosta terrestre nel distretto considerato e, sulla base di altri esempi, ne conclude che le deviazioni dalla verticale non solo sono molto grandi na altresì molto regolari in zone sismiche o vulcaniche. 1)all'esame della Figura 11 possiamo notare che le deviazioni della verticale nella 
Penisola sono fortemente indirizzate verso il sistema degli Appennini, che della Penisola costituiscono l'ossatura assiale.

Poiché sulla costa orientale dell'Adriatico si verifica il fenomeno opposto (flessione verso le Dinariche), da questa diffusa attrazione verso gli Appennini da un lato e verso le Dinariche dall'altro, dobbiamo concluderne che la densità del materiale subcrostale sotto il letto dell'Adriatico è relativamente minore di quella sotto i sistemi

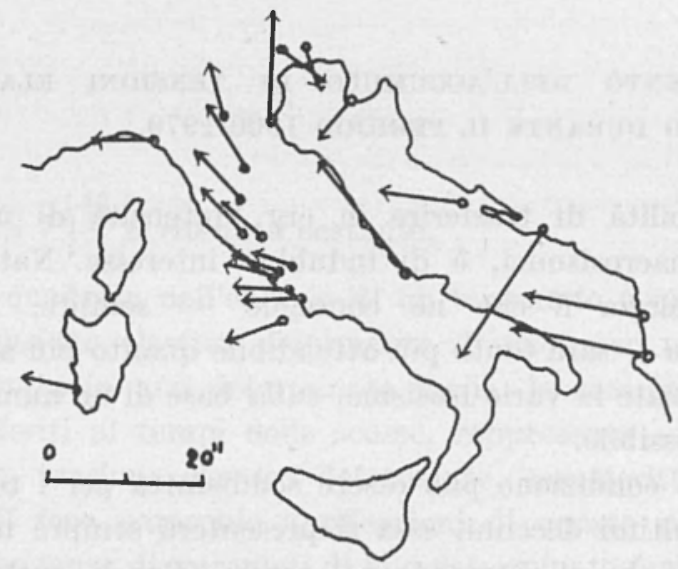

Fix. 11 - Deviazione della verticale nella zona italiana (le frecce sono in senso opposto a quello della deviazione). (Secondo Kawabata).

montuosi posti ai suoi lati. Questa conclusione è in perfetto parallelismo con gli aspetti geodinamici della zona. Abbiamo già notato come, alle dilatazioni ipocentrali nei terremoti dell'Adriatico facciano riscontro le compressioni negli ipocentri dei terremoti appenninici. Ma anche nella velocità di propagazione delle onde elastiche, l'alto Adriatico si presenta anomalo nei confronti della Penisola.

In un lavoro sul terremoto dell'alto Adriatico del 30 Novembre 1934, Caloi (1937) ha provato che ad una leggera diminuzione della velocità delle onde $P$ fa riscontro un sensibile aumento della velocità delle onde $S$ : mentre infatti le prime hanno una velocità di $7.77 \mathrm{~km} / \mathrm{sec}$ (inferiore alla media), le seconde assumono un valore elevatissimo, $4.8 \mathrm{~km} / \mathrm{sec}$. Ciò è in perfetta armonia con le conclusioni tratte dallo studio delle deviazioni della verticale: ad una minore densità del materiale subcrostale adriatico, fa riscontro, nella stessa zona, una relativamente maggiore rigidità, il che spiega l'aumento della velocità delle onde trasversali. Nel caso sopra citato, inoltre il rapporto di Poisson 
assume un valore di $\sigma=0.18$, che corrisponde ad una rigidità nettamente più accentuata di quella normale; non solo, ma altresi comporta una relativamente netta diminuzione di $\lambda$ nei confronti di $\mu$, il che spiega la flessione nel valore della velocità delle onde longitudinali. A conclusioni analoghe è giunto successivamente Valle (1948), nei confronti fra le caratteristiche elastiche della Calabria e dell'adiacente fondo del Mar Jonio.

\section{IV. - ANDAMENTo DELL'ACCuMulo DI TENSIONI ELASTICHE NEL- L'Abruzzo durante IL PERIOdo 1900-1970.}

La possibilità di trasferire in erg l'intensità di un terremoto, noti i dati macrosismici, è di indubbio interesse. Naturalmente, il valore dell'energia $E$ che ne consegue - sempre, forzatamente, approssimativo - sarà tanto più attendibile quanto più accuratamente risultano tracciate le varie isosisme, sulla base di un numero di notizie più grande possibile.

Se questa condizione puo essere soddisfatta per i terremoti verificatisi negli ultimi decenni, essa si presenterà sempre meno sicura a mano a mano che si retrocede nel tempo. Per le antiche cronache, l'operazione più delicata sarà quella di tradurre in gradi della scala Mercalli (originaria o modificata) le notizie, spesso scarse e contraddittorie, dei grandi terremoti del passato. In molti casi però l'operazione merita di essere compiuta, e ne vedremo subito il perchè.

Nel 1940, il giapponese Tsuboi aveva proposto di esprimere l'energia $E$ di un terremoto con la seguente formula

$$
E=\frac{1}{2} \mu \varepsilon^{2} V
$$

dove $\mu$ rappresenta l'effettiva costante elastica della crosta terrestre, $\varepsilon$ la deformazione elastica media precedente il terremoto e $V$ il volume della zona sottoposta a deformazione.

Evidentemente $\varepsilon$, scelto come valor medio, presuppone che il volume del materiale, che sarà interessato dal terremoto, sia uniformemente deformato, in modo che $\varepsilon$ sia dovunque lo stesso all'istante in cui il terremoto si verifica, così che l'energia liberata $E$ provenga dall'intero volume soggetto a deformazione. Si tratta, non c'è dubbio, di un'ipotesi molto restrittiva, di difficile realizzazione. 
Ad ogni modo, questa formula fu ripresa da Hugo Benioff. Messa la [4] sotto la forma

$$
E^{1 / 2}=\left(\frac{1}{\tilde{L}} \mu \nabla\right)^{1 / 2} \cdot \varepsilon
$$

Benioff osservò che, in prima approssimazione, considerate invariabili, per una determinata faglia attiva, le grandezze $\mu$ e $\nabla$, la [5] può scriversi

$$
E^{1 / 2}=C \cdot \varepsilon
$$

dove $C=\left(\frac{1}{2} \mu V\right)^{1 / 2}$ è ritenuta costante.

La radice quadrata dell'energia di un terremoto è quindi proporzionale alla tensione elastica deformante. Perciò, per una sequenza di scosse avvenuta in una determinata faglia, la somma degli incrementi $E_{i}{ }^{1 / 2}$, riferiti ai tempi delle scosse, rappresenta - a meno del fattore $C$ - la tensione elastica deformante, caratteristica della sequenza. Bemioff fece parecchie applicazioni di questo principio, non solo a singole sequenze di terremoti di una determinata faglia, ma anche a sequenze di terremoti, verificatisi nel mondo con determinate profondità, con determinate magnitudo, ecc.

Questo metodo può essere utilizzato vantaggiosamente anche nella determinazione della sismicità di un distretto sismico, potendo usufruire dei dati macrosismici ivi raccolti durante uno o più secoli. L'interesse di queste determinazioni (a prescindere dalla loro limitata approssimazione) è quello di fornire indicazioni sull'accumulo di tensioni attuale, nel tratto di crosta terrestre investigato (Fig. 12).

A questo proposito, recentemente è stata persino adombrata la possibilità di abbassare l'entità della tensione in fase di accumulo in una determinata zona sismica. Cesare Emiliani, Christopher Harrison e Mary Swanson dell'Università di Miami, studiando gli effetti di recenti esplosioni nucleari sotterranee, provocate nel Nevada e in altre regioni degli Stati Uniti, trovarono che tali esplosioni suscitano, a loro volta, una sensibile attività sismica per almeno 32 ore dopo l'evento e fino a distanze di almeno $860 \mathrm{~km}$. A conclusioni analoghe pervennero R. M. Hamilton, F. A. Me Keown e J. H. Healy dell'“ U.S. Geological Survey ». Poiché, più a lungo le tensioni si accumulano in una zona sismica, più pericolosa è da attendersi la susseguente attività sismica, 
Ismiliani e collaboratori suggeriscono l'opportunità di impiegare esplosioni nucleari sotterranee allo scopo di liberare la litosfera dalle tensioni ivi accumulate, per deformazione tettonica naturale. Periodiche esplosioni in zone sismicamente attive potrebbero essere usate per prevenire disastrosi terremoti. I)i qui l'interesse di conoscere l'andamento dell'accumulo delle tensioni elastiche in dette zone (come da Fig. 12).

Nel caso specifico, abbiamo tracciato il diagramma di $\Sigma_{i} E^{1 / n}$ per il periodo preso in esame, associato alla sismicità della zona abruzzese. Ja Fig. 12 non richiede estesi commenti. Si osserva che quanto più lıngo è il periodo di relativa quiete sismica di una regione, tanto, magrgiore è generalmente l'accumulo delle tensioni elastiche e, di conseguenza, il pericolo di terremoti disastrosi.

Il risultato esposto, naturalmente, è di grossolana approssimazione. In queste ricerche - almeno allo stato attuale delle nostre conoscenze, è illusorio perseguire valori assoluti. Ciò che più preme ̀̀ conoscere l'andamento delle variazioni, e l'approssimazione di questo è strettamente legata all'uniformità del metodo seguito.

N.B. - I dati macrosismici sono stati dedotti da cataloghi diversi; si è ritenuto superfluo far stampare le isosisme.

\section{BIBLIOGRAFIA}

Accons I B., La componenle traslativa nella tettonica dell'A ppennino lazialeabruzzese. "Geologica Romana ", V (1966).

Bknko E., Appunti geologici sulle regioni dell'Appennino centrale, compreso nel joglio 151 (Alatri). "Boll. Uff. Geol. d'Italia ", 63 (1938).

Beneo E., Sezione geologica della penisola italiana dal Tirreno all'Adriatico. "Boll. Uff. Geol. d'Italia », 64 (1939).

Brnko E., Schema tettonico dell'Abruzzo Nord-Orientale. "Boll. Uff. Geol. d'Italia n, 68 (1943).

Bxioff II., Global Strain Sequence of Great Shallow Earthquakes. "Bull. Geol. Soc. An1. ", 62 (1951).

Caior P., Il terremoto adriatico del 30 Novembre 1934. "Boll. Soc. Sismol. Ital. n, XXXV (1937).

Calor P., Struttura geologico-sismica dell'Europa centro-meridionale, dell' Italia $e$ del Mediterraneo centro-occidentale, quale risulta da recenti ricerche condotte in Italia. "Ann. di Geof.", V (1952).

Caror P., On the Upper Mantle. In "Advances in Geophysics 12, Academic Press, New York (1967). 

DENANGEOT Y., L'are Abruzzais externe. Ftud tectonique. a Contributi di Scienze geologiche ", II. In "La ricerea scientifica ", Anno 220 (1952).

D) Filippo D). Marcelli L., Uno studio del terremoto del Gran Sasso d'Tlalia del is Settembre 1950, "Ann, di Geof, ", IV (1951).

DI Filippo D.-MARcelli L., Struthura della erosta terrestre in corrispondenza dell'Italia Centrale. "Ann. di Geof. n, V (1952).

Di Filippo 1).-Pynonaci F., La sismicità del enmpo fagliato dell'Aterno. "Ann, di Geof. ", XVII (1964).

Emiliant C., Iratzison C. G. A., Swanson MI, Underground Nuclear Explosions and the Control Earthquakes. "Seience", 165, N. 3899 (1969).

Fabiani R., Segte A., Schema strutturale della Regione Italiana. "Contributi di Seienze Geologiehen, II. In "Ja Ricerca Scientifica ", (Anno 220 1952).

(iUtenderg B., Earliquake magnitude, intensity, energy and acceleration. "Bull. Seis. Soc. Am, ", 34 (1942).

Hanilton R. M., McKeown F. A., IIEaly Y. H., Sesmic delivily and Faulting Associated with Large Linderground Nuclear Explosion. "Scien. ce $n, 166$, N. 3905 (1969).

Kаялік $\nabla$., Magnihudebestimmung europäizeher Nahbeben. "Traviux Inst. Géophys. Aearl. Tehécosl. d. Se. ", 47, Praha (1957).

Kawabata Y., A Study on the Deviation of the Plumbo-Line in Japan "The Geophys. Mag. ", 26 (1954).

Marcelli J.-Pannoccira G., L'atività sismica nella zona delle Sorgenti del Peschiera. Parte I. "Ann. Ili Geof. "XVI (1963).

Tsuror C., Isostasy and Maximum Earthquake Energy. "Proc. Inp. Acul. Yapau n, 16 (1940).

VALLE P. E., Contributo allo studio delle caratteristiche sismiche del Mediterraneo centro-orientale, "Amn. di Geof. ", I (1948). 